\title{
Large time behavior and asymptotic stability of the two-dimensional Euler and linearized Euler equations
}

\author{
Freddy Bouchet**** and Hidetoshi Morita*
}

October 31, 2018

\begin{abstract}
Affiliations : * INLN, CNRS, UNSA, 1361 route des lucioles, 06560 Valbonne, France
** CNLS, Los Alamos National Laboratory, PO Box 1663, Los Alamos, NM 87545, United States

Corresponding author : Freddy Bouchet ; email : Freddy.Bouchet@inln.cnrs.fr
\end{abstract}

Keywords : 2D Euler equations, Large scales of turbulent flows, 2D turbulence, geophysical turbulence, asymptotic behavior, asymptotic stability.

\begin{abstract}
We study the asymptotic behavior and the asymptotic stability of the two-dimensional Euler equations and of the two-dimensional linearized Euler equations close to parallel flows. We focus on flows with spectrally stable profiles $U(y)$ and with stationary streamlines $y=y_{0}$ (such that $U^{\prime}\left(y_{0}\right)=0$ ), a case that has not been studied previously. We describe a new dynamical phenomenon: the depletion of the vorticity at the stationary streamlines. An unexpected consequence, is that the velocity decays for large times with power laws, similarly to what happens in the case of the Orr mechanism for base flows without stationary streamlines. The asymptotic behaviors of velocity and the asymptotic profiles of vorticity are theoretically predicted and compared with direct numerical simulations. We argue on the asymptotic stability of this ensemble of flow profiles even in the absence of any dissipative mechanisms.
\end{abstract}

\section{Introduction}

The flow of a perfect fluid is described by the Euler equations, one of the oldest equations of mathematical physics [28. Four century after their discovery by Euler, these equations still propose big challenges both to mathematician and physicists [28]. Two-dimensional flows and the two-dimensional Euler equations are mathematically much simpler than their three dimensional counterparts, but still present some very interesting unsolved problems. One of the main phenomena for two-dimensional flows is the self-organization into coherent structures [44, 54, 43, 64, 59, 42]: monopoles, dipoles, and parallel flows. Such large-scale structures are analogous to geophysical cyclones, anticyclones, and jets in the ocean and atmospheres. This analogy, understood thanks to the theoretical strong similarities between the 2D Euler equations on one hand and the Quasi-Geostrophic or the Shallow Water models on the other hand, is one of the main motivations for the study of the 2D Euler equations. The 2D Euler equations also describe experimental flows: the transverse dynamics of electron plasma columns [57, the dynamics of fluids when three-dimensional motion is prevented by a strong transverse field (rotation, a transverse magnetic field in a liquid metal, etc. 64]) or the dynamics of fluids in very thin geometries [51].

Because large-scale coherent flows appear spontaneously in two dimensional turbulence, their stability is a crucial problem. Moreover, the study of the dynamical mechanism that describes the relaxation towards these stable flows is essential. In this paper, we consider stable parallel base flows $\mathbf{v}_{0}(x, y)=U(y) \mathbf{e}_{x}$, which are dynamical equilibria of the 2D-Euler equations. We prove that the velocity of these flows is asymptotically stable, that is that all the solutions to the nonlinear Euler equations that start near $\mathbf{v}_{0}$ converge to some other parallel flows $\mathbf{v}_{0}+\delta U(y) \mathbf{e}_{x}$, close to $\mathbf{v}_{0}{ }^{1}$. Our analysis mostly relies on the linearization of the Euler equations close to the base flows, which we prove to actually describe also the nonlinear relaxation at leading order. More

\footnotetext{
${ }^{1}$ This notion of convergence makes the notion of asymptotic stability stronger than the alternative Lyapounov stability, that only states that all solutions of the nonlinear equations that start near a steady point $\mathbf{v}_{\mathbf{0}}$ stay near $\mathbf{v}_{\mathbf{0}}$ forever. The asymptotic stability of the velocity refers to asymptotic stability in the kinetic energy norm.
} 
precisely, we prove that the perturbation velocity decays algebraically for large times. As far as the linearized dynamics is concerned, an important improvement over the previous works is the understanding of the case when the flow has some stationary streamlines $y=y_{0}$ (or equivalently the velocity profile $U(y)$ has some stationary points $\left.y_{0}, U^{\prime}\left(y_{0}\right)=0\right) 2$, which has not been elucidated even qualitatively previously.

Besides the stability and the asymptotic stability problem itself, the evolution operator for the linearized Euler equations plays a very important role in different statistical approaches to turbulent flows [24, 21, 31. Indeed, in a turbulent context, it is likely that the qualitative or quantitative properties of the fluctuations around such stable structures are related to the linearized dynamics. For instance, in quasi-linear approaches or second order closure of the evolution of the Euler equations, point vortex model [24], or of the Navier-Stokes equation (either forced or unforced, and either deterministic or stochastic,) the linear operator appears naturally as an essential theoretical tool. Similarly, in the forced problems, in the linear regime, the response can be easily expressed in terms of the evolution operator for the linearized dynamics or of the resolvent operator for the dynamics. The behavior of such operators for large times is thus a very important issue that has many theoretical and dynamical consequences. In this work, we quantify very precisely the large time asymptotic behavior of the evolution operator for the linearized 2D-Euler equations, and discuss briefly some of the implications for the above problems.

The stability of the large-scale coherent structures of two-dimensional flows is a very old and classical field of fluid mechanics. For instance Rayleigh [53, Kelvin 65], Orr [50, Sommerfeld 63, and many other famous scientists from the nineteenth and the beginning of the twentieth century have participated to the understanding of the linear theory for the 2D Euler equations close to parallel flows. Mathematicians gave also important contributions: Arnold's theorems [2] and some modern generalizations [69, 61, 26, 18, 10] prove the Lyapounov stability of some of these flows. Even if this work deals only with the behavior of slightly perturbed stable flows, other equally interesting and important problems arise in the study of unstable or oscillatory flows. Recently many works have been devoted to the proof of the instability of some classes of flows, the characterization of the spectrum of the linearized equations, and some estimates on the stability and the instability of such flows ; see for instance [32, 60, 37, 8] and references therein.

Our work is based on the linearized 2D Euler equations. The Rayleigh equation [53], which describes modes for the linear dynamics, has been a subject of mathematical and theoretical research since the beginning of the twentieth century [23, and is still currently active. The main interest lies in the dynamical phenomena associated with the singularities at the critical layers (the singularities appearing when the frequency of the perturbation is equal to that of a closed streamline of the base flow). However, the modes of the Rayleigh equation do not describe the fully linearized dynamics, because the linear operator is non-normal [30]. Among the peculiarities of the linearized Euler equations, we stress the Orr mechanism [50]: the base flow shears the perturbation producing thinner and thinner filaments ; then when the velocity or the streamfunction is computed, the effect of such filaments being smoothed out, the perturbation velocity decays for large times. This mechanism is easily quantified when the shear is linear, for the Euler equations [50] or using for instance Kelvin waves [65] for viscous flows. Case [19] and Dikii [22] were the first to stress that in general, for inviscid flows, such a phenomenon is outside the scope of a modal description using the Rayleigh equation. When the shear is linear, using a Fourier-Laplace transform, the dynamics of the perturbation is properly described in the framework of an initial value problems. They concluded that the perturbation velocity decreases asymptotically with an algebraic law for large times. Other phenomena associated to the non-normality of the linear operator include possible transient growth [29, 68, 1], inviscid damping (the counterpart of Landau damping in plasmas), axisymmetrization [56, 40], and algebraic instabilities [49]. From a mathematical point of view, the singularities at the critical layers lead to the existence of a continuous spectrum for the linearized Euler equation. The analysis of the properties of this continuous spectrum explains most of these transient growth, inviscid damping, algebraic instabilities, and so on.

From a theoretical point of view, one class of works used the Laplace transform tools [5, 4, 57, following the initial works of Case [19], Dikii [22] and the generalization to non-uniform shear by Briggs-Daugherty and Levy [12. Another class of studies, less general but very enlightening, used simple or particular base flows or special conditions for which explicit computations are possible [65, 50, 70, 29, 66, 14, 15, 16, 49].

In this paper, we are especially interested in the precise description of the large time asymptotic behavior of the 2D Euler and 2D linearized Euler equation close to parallel base flows. For the linearized dynamics of stable base flows, once the contribution of possible neutral modes has been subtracted, the asymptotic behavior is related to the continuous spectrum of the linearized operator. In the case of the base flow with a linear shear,

${ }^{2}$ Please note that the profile $U(y)$ has stationary points, but the two-dimensional base flow has no stationary points 
$U(y)=\sigma y$, the explicit computations by Case 19 showed that, due to the Orr mechanism, for large times the velocity perturbation decays algebraically :

$$
v_{x} \underset{t \rightarrow \infty}{\sim} \frac{C(y)}{t^{\alpha}} \text { and } v_{y} \underset{t \rightarrow \infty}{\sim} \frac{C(y)}{t^{\beta}}
$$

with exponents $\alpha=1$ for the longitudinal component $v_{x}$ of the velocity perturbation and $\beta=2$ for the transverse one $v_{y}$.

For more general base flows with strictly monotonic profiles $U(y)$ (without stationary streamline), it is a common belief that the exponents $\alpha=1$ and $\beta=2$ remain valid. This belief is based on the results of an ansatz for large time asymptotics [13] (see also [4], appendix A). Some interesting comments about the temporal behavior of the streamfunction and velocity, in the case of localized initial perturbations (vorticity defects), can also be found in [4 section 7. Even if we have not found any complete rigorous proofs, very precise classical arguments using the Laplace transform [55, 12] conclude that the contribution of the continuous spectrum to the stream function perturbation $\psi$ decays with $\psi \underset{t \rightarrow \infty}{=} \mathcal{O}\left(\frac{1}{t}\right)$, in agreement with equation (11). However, these arguments do not generalize where the profile $U(y)$ is not monotonic (flows with stationary streamlines).

From Lundgren work (41], appendix A), we see that the preceding algebraic decay for the velocity or the streamfunction may be related to the following asymptotic behavior for the perturbation vorticity:

$$
\omega(y, t) \underset{t \rightarrow \infty}{\sim} \omega_{\infty}(y) \exp (-i k U(y) t)+\mathcal{O}\left(\frac{1}{t^{\gamma}}\right)
$$

where $k$ is the initial perturbation wave number. Indeed, computing the velocity from Lundgren's ansatz (equation (2) ) and assuming uniformity in the asymptotic expansion, we obtain oscillating integrals leading to algebraic decay for large times. The values for the exponents $\alpha=1$ and $\beta=2$ are then related to the singularities of the Green function used in order to compute the velocity perturbation from the vorticity perturbation. This argument, assuming Lundgren's ansatz, suggests that the asymptotic behavior for the velocity should be different for velocity profiles $U(y)$ with stationary points $y_{0}\left(U^{\prime}\left(y_{0}\right)=0\right.$, base flow with stationary streamline). Actually, in such a case, the stationary phase asymptotics for oscillating integrals would generically give $1 / \sqrt{t}$ contributions. It has then been noticed by several authors, that with such a $1 / \sqrt{t}$ law, Lundgren's ansatz would be self-consistent. [13, 16, 14]. Similar problems have also been noticed by Brown and Stewartson [13], as their own asymptotic expansion clearly breaks down where $U^{\prime}(y)=0$ (base flows with stationary streamlines). Besides Lundgren and Stewartson, many authors have insisted on the specificity of base flows with stationary streamlines (see for instance [16, 14, 38]).

In the past, there have been only a few studies considering base flows with stationary streamlines. In the case of the equations for 2D barotropic flows on a $\beta$ plane (a direct generalization of the 2D Euler equations), Brunet and coauthors [16, 14, have studied the dynamics close to a parabolic jet when the potential vorticity gradient exactly cancels the $\beta$ effect. This case is similar to the linear shear case in the Euler equations studied by Kelvin, Orr, Case and others [65, 50, 19, 66, 29], in that the vorticity gradient exactly cancels out, which makes the linearized equation much simpler and amenable to a very interesting explicit analytic treatment. In the following, we will argue that, because of the cancelation of the vorticity (or potential vorticity) gradient, the dynamics of these cases is actually non generic, and that flows where the vorticity (or potential vorticity) gradient does not vanish behave differently.

In the general case, the asymptotic behavior of the vorticity and velocity perturbations of flows with stationary streamlines thus remains unstudied. In natural flows, however, jet velocity profiles are most of time not monotonic but have some extrema, i.e. the flow has stationary streamlines ; see for instance Jupiter, atmospheric, and ocean jets. Why have cases with stationary streamlines not been studied previously? This may be partially on account of the wrong belief that base velocity profiles with stationary points should be unstable. It is true that many of the flows with extrema in their velocity profiles do not fulfill the classical Rayleigh-Fjørtoft criteria [23]. However, these criteria are only sufficient conditions of stability. Moreover, as seen in natural flows and as shown bellow with several examples, many parallel flows with stationary streamlines and not fulfilling the classical Rayleigh-Fjørtoft criteria are actually stable. Another reason for a lack of studies may also be the theoretical difficulty with Laplace tools in this case, related to the presence of stationary streamlines (merging of critical layers). Indeed, an essential tool for the Laplace transform is the analytic continuation of dynamical quantities, performed by avoiding the singularities associated to the critical layers, with the use of integration in the complex plane [23]. As will be discussed bellow, in the case of flows with stationary streamlines, in order 
to perform the analytic continuation, one would need to find a path in the complex plane passing at the same time above and bellow the singularity, which is clearly impossible. For this reason, it has often been stated that Laplace tools cannot be used when stationary streamlines are present. By contrast, we illustrate in this work that even if analytic continuation can not been performed in this way, Laplace tools are still very useful and lead to very interesting results.

In the following we consider the generic case of a parallel flow with any profile, either with or without stationary streamlines, improving by far the class of previously studied flows and overcoming the previously discussed difficulties. We also discuss possible generalizations to monopole vortices. We show how the Laplace transform is generalized to the case of base flows with stationary streamlines. For instance, we show how the classical determination of the number of unstable modes, by using Nyquist's plots, remains valid in this case. From this general theoretical approach, we prove that the asymptotic vorticity field actually follows the Lundgren's ansatz (2), even in the case of a base flow with stationary streamlines. Similarly the velocity field decreases also algebraically with the power laws (1), with $\alpha=1$ and $\beta=2 \sqrt{3}$. This may seem paradoxical, after the discussion of the preceding paragraphs. Actually, the naturally expected $1 / \sqrt{t}$ contributions from the stationary phase asymptotics do not exist, unexpectedly. One reason is the non-uniformity of the asymptotic expansion in Lundgren's ansatz. Another more important reason is related to a new dynamical phenomena leading to the rapid decrease and cancelation of the vorticity perturbation exactly at the stationary streamline (see Fig. 11), which partially erase the effect of the stationary phase. We call this phenomena vorticity depletion at the stationary streamlines. This is a non-local collective phenomena, due to the effect of the perturbation velocity on the background vorticity gradient. For this reason, this phenomena has not been observed in the previous studies involving stationary streamlines [16, 14, 38, because these cases have an exactly zero vorticity (or potential vorticity) gradient. These last cases are thus non generic.

In the following, we predict the vorticity depletion at the stationary streamlines using Laplace tools. It is thus a generic effect in any type of parallel flow with non-monotonic stable velocity profile. We also illustrate the results by direct numerical simulations in the case of Kolmogorov base flow $U(y)=\cos y$, for the $2 \mathrm{D}$ Euler equations with periodic boundary conditions. This vorticity depletion mechanism also impacts non linear turbulent flows when the perturbations are small enough to be governed by the linearized equations, as discussed in the conclusion.

We establish the large time asymptotic contributions to the vorticity and to the velocity fields, by the continuous spectrum, in the case of the linearized dynamics. We are then able to discuss the asymptotic stability of the velocity of parallel base flows, for the non-linear dynamics. The result is that parallel base flows which have no mode (neither stable nor unstable) are asymptotically stable: any small perturbation leads to a small deformation of the base flow, the perturbation velocity to this new base flow decaying algebraically. Stable Kolmogorov flows are examples of base flows without modes, illustrating the importance of this class of flows. Based on these results we also conclude that a quasi-linear approach predicts the asymptotic velocity profile. We note that all this is true only thanks to a non-trivial cancelation of leading order terms, already noted by 41. The current work put the validity of a quasi-linear approach on a more rigorous ground, and proves that this it is also valid also for flows with stationary streamlines. It also gives an efficient theory and numerical tool to predict the asymptotic flow.

For the case of a circular vortex base flows, the work [7] shows that the far field velocity decays with exponents different from the case of parallel flows. For circular vortex base flows, an example of non-monotonic angular velocity profile has also been studied [49], based on a special explicit solution 62. This example shows a very interesting algebraic instability with $t^{1 / 2}$ growths. Even if we do not explicitly treat the case of stable circular vortices $\mathbf{v}(r)=U(r) \mathbf{e}_{\theta}$ in the present study, the generalization to this case of the present study could be taken following similar theoretical arguments. The mechanism of vorticity depletion at stationary streamlines or at the core of stable probably also exist for stable vortices, as discussed in the conclusion.

In section 2 we introduce the 2D Euler equations and the linearized Euler equations. Section 2 describes the theory related to the linearized Euler equation. We discuss the main results related to the asymptotic behavior of the vorticity and velocity fields in section 2.2 The core of the proof relies on the results of the analysis on the limit of small $\epsilon$ for the resolvent operator in section 2.3 .

Section 3 discusses the asymptotic stability of parallel flows for the Euler equation.

\footnotetext{
${ }^{3} \mathrm{An}$ exception is the velocity field close to the stationary streamline, where we have no theoretical prediction, but where we observe numerically that either $\alpha=1$ and $\beta=2$ or $\alpha=\beta=3 / 2$ depending on the symmetry of the perturbation.
} 


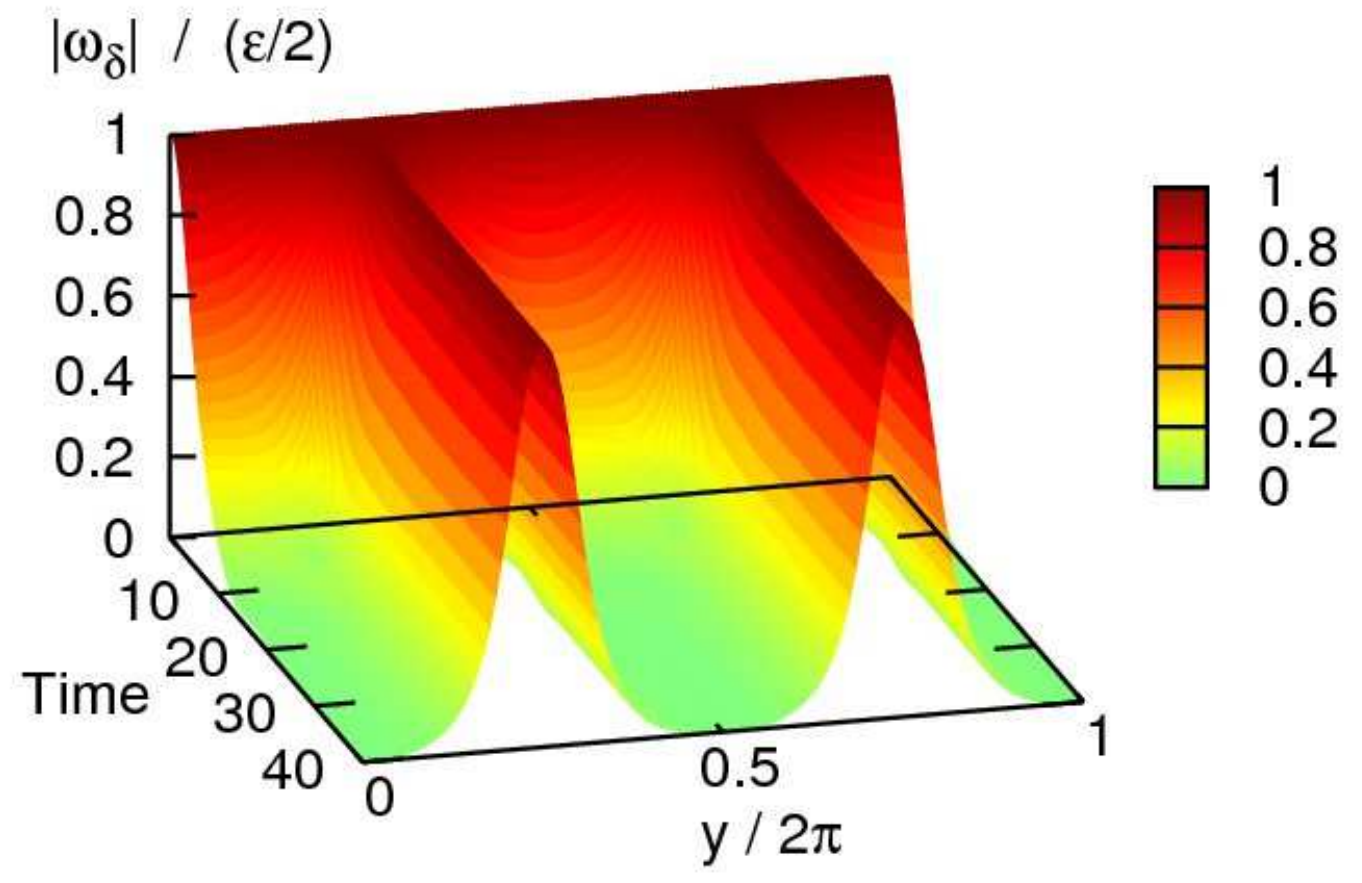

Figure 1: Evolution of the vorticity perturbation $\omega(x, y, t)=\omega(y, t) \exp (i k x)$, close to a parallel flow $\mathbf{v}_{0}(x, y)=$ $U(y) \mathbf{e}_{x}$ with $U(y)=\cos (y)$. The figure shows the modulus of the perturbation $|\omega(y, t)|$ as a function of time and $y$. One clearly sees that the vorticity perturbation rapidly converges to zero close to the points where the velocity profile $U(y)$ has extrema $(y=0$ and $\pi)$. This depletion of the perturbation vorticity at the stationary streamlines is a new generic self-consistent mechanism, understood mathematically as the regularization of the critical layer singularities at the edge of the continuous spectrum. 
These results are illustrated in the case of doubly periodic boundary conditions, with the Kolmogorov flows as a base flow, in section 4 . For some aspect ratio, these flow are stable even if they do not fulfill the hypothesis of any of the two Arnold's theorems. Applying Arnold's ideas to this case, we first prove their Lyapounov stability in section 4.1. In section 4.2, we show the results of direct numerical simulations of the 2D Euler equations (nonlinear), which both illustrate the theoretical results of section 2, and show that the linearized dynamics correctly describes the nonlinear one.

Section 5 discusses briefly some consequences of these results for the 2D Euler and Navier-Stokes equations with stochastic forces and for possible theories relying on a quasi-linear or kinetic approach. It also discusses some possible generalizations to models of interest for geophysical flows.

\section{The 2D Euler and linearized Euler equations}

Let us consider the 2D Euler equations

$$
\frac{\partial \Omega}{\partial t}+\mathbf{V} \cdot \nabla \Omega=0
$$

where $\Omega$ is the vorticity and $\mathbf{V}$ is the velocity. We consider this equation either in an infinite plane, in a channel geometry with boundary conditions $\mathbf{V} \cdot \mathbf{n}=0$ on the boundary wall, or on a doubly periodic domain $(0,2 \pi / \delta)(0,2 \pi)$, where $\delta>1$ is the aspect ratio. In some parts of the discussion, for technical reasons the boundary conditions will be important. Then only the case of a doubly periodic domain will be explicitly treated. However all the results are applicable to the channel and infinite domain geometries with slight modifications.

We study the asymptotic stability of parallel flows $\mathbf{v}_{0}=U(y) \mathbf{e}_{x}$. We will thus consider the Euler equations (3) with initial conditions close to this base flow : $\Omega=\omega_{0}+\omega$ and $\mathbf{V}=\mathbf{v}+\mathbf{v}_{0}$, where $\omega_{0}(y)=-U^{\prime}(y)$ is the base flow vorticity and $\omega$ and $\mathbf{v}$ are the perturbation vorticity and velocity, respectively.

We also need to consider the linearized 2D Euler equations close to this base flow. It reads

$$
\frac{\partial \omega}{\partial t}+\mathbf{v} \cdot \nabla \omega_{0}+\mathbf{v}_{0} \cdot \nabla \omega=0
$$

We assume that the base flow $U(y)$ has no unstable mode (a precise definition of modes will be given along the discussion). In section 4 we will illustrate some of the results on the particular case of the Kolmogorov flow $U(y)=\cos y$ (in a doubly periodic domain).

\subsection{The Laplace transform, resolvent operator and Rayleigh equation}

In this section and the following we consider the linearized 2D Euler equations. We give the main definitions used later on.

We decompose the perturbation vorticity in Fourier series for the $x$ variable only. For parallel flows, due to the translational invariance, these Fourier modes are independent one from the others for the linear dynamics. In the following, we thus study perturbations of the form $\omega(x, y, t)=\omega_{k}(y, t) \exp (i k x)$ and $\psi(x, y, t)=\psi_{k}(y, t) \exp (i k x)$, where $\psi$ is the stream function, with $\omega=\Delta \psi$ and $k$ is the longitudinal wave number. In the following, we drop the $k$ subscripts for the perturbation. The relations between $\omega, \mathbf{v}$ and $\psi$ are then

$$
\omega=\frac{d^{2} \psi}{d y^{2}}-k^{2} \psi, \quad v_{x}=-\frac{d \psi}{d y} \text { and } v_{y}=i k \psi .
$$

The linearized Euler equations then reads

$$
\frac{\partial \omega}{\partial t}+i k U(y) \omega-i k \psi U^{\prime \prime}(y)=0
$$

We will study the long time asymptotics of the linearized equation. The more general approach is to use the Laplace transform of equation (6). We define the Laplace transform $\hat{\omega}$ of $\omega$ as

$$
\hat{\omega}(y, p)=\int_{0}^{\infty} d t \omega(y, t) \exp (-p t)
$$


The Laplace transform is analytic for any complex number $p$ for sufficiently large real part $\Re p$. The inverse Laplace transform is given by

$$
\omega(y, t)=\frac{1}{2 \pi i} \int_{\Gamma} d p \hat{\omega}(y, p) \exp (p t)
$$

where the complex integration is performed along a Bromwich contour $\Gamma$ in the complex plane of $p$. In the following we use the notation $p=-i k(c+i \epsilon)$ where $c$ and $\epsilon$ are real numbers; $c$ and $\epsilon$ are homogeneous to velocities. We assume $k>0$. The Laplace transform $\hat{\omega}$ is thus analytic for sufficiently large $\epsilon$.

The Laplace transform of equation (6) reads

$$
(U(y)-c-i \epsilon) \hat{\omega}-U^{\prime \prime}(y) \phi=\frac{\omega(y, 0)}{i k},
$$

where $\phi=\hat{\psi}$ is the Laplace transform of $\psi$ and $\omega(y, 0)$ is the initial value for the vorticity field. We have

$$
\hat{\omega}=\frac{d^{2} \phi}{d y^{2}}-k^{2} \phi
$$

From a mathematical point of view, we have to solve the equation for $\phi$

$$
\left(\frac{d^{2}}{d y^{2}}-k^{2}\right) \phi-\frac{U^{\prime \prime}}{U-c-i \epsilon} \phi=\frac{\omega(y, 0)}{i k(U-c-i \epsilon)},
$$

with the boundary condition for $\phi$ (here $\phi$ doubly periodic). The solution of this boundary value problem $\phi[\omega(., 0)](y, c+i \epsilon)$ depends functionally on the initial value of the vorticity $\omega(y, 0)(\phi$ is the resolvent operator for the stream function). This resolvent operator encodes all the information about the temporal evolution of the stream function and the vorticity field.

The homogeneous part of equation (9) is the celebrated Rayleigh equation. It is also the equation for modes $(\psi=\phi(y) \exp [i k(x-(c+i \epsilon) t)])$ of the linearized Euler equation (4). It reads

$$
\left(\frac{d^{2}}{d y^{2}}-k^{2}\right) \phi-\frac{U^{\prime \prime}}{U-c-i \epsilon} \phi=0
$$

with the flow boundary conditions. For neutral modes $(\epsilon=0)$, this is a non-classical boundary value problem, because of the possible singularities associated to the vanishing of $U-c$. Any $y_{c}$ such that $U\left(y_{c}\right)=c$ is called a critical point for the velocity $c$. For any $c$, the free motion on the streamline $y=y_{c}$ is called the critical layer and has exactly the frequency $k c$.

Any $y_{0}$ such that $U^{\prime}\left(y_{0}\right)=0$ (no shear, for instance for velocity extrema) is called a stationary point of the jet profile, corresponding to a stationary streamline. We then call $c_{0}=U\left(y_{0}\right)$ a stationary velocity. If $y_{0}$ is a local extrema of $U$, we note that when $y \rightarrow y_{0}$ (or equivalently $c \rightarrow c_{0}=U\left(y_{0}\right)$ ), two critical layers, one on each side of the velocity extrema, merge on a single one.

The range of the profile $U$ is the ensemble of velocities $c$ such that $\min _{y} U(y)<c<\max _{y} U(y)$.

In the following we assume that the base flow is spectrally stable, i.e., no unstable mode exist, which means that no solution to (10) exist for any $c+i \epsilon$ with strictly positive $\epsilon>0$. Then, as shown in section 2.3, equation (9) has a unique solution for any $c+i \epsilon$ with strictly positive $\epsilon>0$. We also assume that no neutral mode exist, which means that no solutions to (10) is found in the limit $\epsilon \rightarrow 0^{+}$(a more precise definition will be given bellow in terms of the dispersion relation). This no mode assumption for equation (6) may seem strange, but it is indeed a generic situation when such flows are stables. It is indeed a classical result that shear flow without inflection points, or vortex with strictly decreasing vorticity profile are stable and have no neutral mode [23, 12]. For instance, in section 4, we prove that this hypothesis is also verified for the Kolmogorov flow $U(y)=\cos (y)$ as soon as the aspect ratio $\delta$ is larger than 1 . Actually the only examples we are aware of, of stable flows for the 2D Euler dynamics, with neutral modes that remain when we add small perturbation to the flow, are cases with localized vorticity profile [57]. Usually modes appear, when a parameter is changed, at the edge of an instability ; four unstable (or two degenerate) unstable modes eigenvalue then emerge from the continuous spectrum.

The case with neutral modes could be treated following the same lines as what will be discussed bellow; one would then have to separate the contributions by the neutral modes from the contributions by the continuous spectrum. The following discussion analyzes the contributions by the continuous spectrum only. 


\subsection{Large time asymptotic for the linearized Euler equation}

In this section, we predict the large time asymptotic of the linearized Euler equation, using Laplace transform tools. We prove results (11) and (2) and the mechanism of vorticity depletion at stationary streamlines. The heart of the proof relies on the study of the effect of critical layers, on the inhomogeneous Rayleigh equation (9), in the limit $\epsilon$ goes to zero. This rather technical part is performed in section (2.3).

The results of section (2.3) are that the resolvent streamfunction $\phi$, solution of (9), has a finite limit for small positive $\epsilon$ :

$$
\phi(y, c+i \epsilon) \underset{\epsilon \rightarrow 0^{+}}{\rightarrow} \phi_{+}(y, c)
$$

even if singularities exist due to the critical layers. We prove that for any $y, \phi_{+}(y, c)$ is twice differentiable with respect to $c$, except for velocities $c$ that are in the interior of the range of $U$. In this last case, for velocities $c$ that are not stationary, $\phi_{+}$is twice differentiable, except for $c=U(y)$. For $c=U(y)$, $\phi_{+}$is continuous but not differentiable there, and has a logarithmic singularity: for fixed $y, \phi_{+}(y, c)=\Delta \phi_{c}(c-U(y)) \log (c-U(y))+$ $R(y, c)$, where $R(y,$.$) is an analytic function of c$. When $c=c_{0}$ is a stationary velocity, $\phi_{+}\left(y, c_{0}\right)$ is differentiable with respect to $c$.

We think that all of the steps of these proofs could be easily made rigorous from a mathematical point of view, by making explicit the required hypothesis. An exception is for the limit of $\phi$ in the case of critical layers for stationary points. We actually prove in the following that a limit solution exist for $\epsilon=0$, but we do not prove the convergence to it when $\epsilon \rightarrow 0$. In order to deal with this small gap in the proof, we will show, by numerically computing $\phi(y, \epsilon)$, that this convergence actually takes place.

These results (the limit and its properties) are the difficult aspects of the discussion, from a mathematical point of view. Their technical proof can be skipped at a first reading, the next sections can be read independently by assuming these results. The discussion then follows by performing the inverse Laplace transform and proving results (1) and (2) in sections 2.3.4 and 2.3.5.

\subsection{Limit for $\epsilon \rightarrow 0^{+}$of the resolvent operator}

\subsubsection{The dispersion relation}

The equation defining the resolvent operator for the stream function (9) is of the type

$$
\frac{d^{2} \phi}{d y^{2}}+q(y) \phi=f(y)
$$

with $q=-k^{2}-U^{\prime \prime} /(U-c-i \epsilon)$ and $f=\omega(y, 0) /[i k(U-c-i \epsilon)]$. This is a boundary value problem. In order to be precise, we treat the case of a doubly periodic domain with the period $2 \pi$, although that is easily generalized to the case of a flow in a channel $y \in(a, b)$ with the boundary conditions $\phi(a)=\phi(b)=0$.

For $\epsilon \neq 0$, the differential equation is not singular. We consider the homogeneous equation

$$
\frac{d^{2} \phi}{d y^{2}}+q(y) \phi=0
$$

We consider two independent solutions to (13) : $\phi_{1}$ is defined by $\phi_{1}(0)=1$ and $\phi_{1}^{\prime}(0)=0$, and $\phi_{2}$ is defined by $\phi_{2}(0)=0$ and $\phi_{2}^{\prime}=1$ (here and bellow, primes are derivatives with respect to $y$ ). The classical variation of the parameter computation then insures that a particular solution to (12) is

$$
\phi_{p}(y)=-\left(\int_{0}^{y} \phi_{2} f\right) \phi_{1}(y)+\left(\int_{0}^{y} \phi_{1} f\right) \phi_{2}(y)
$$

and a general solution is

$$
\phi_{f}=\phi_{p}+a \phi_{1}+b \phi_{2}
$$

where $a$ and $b$ are unknown constants. The necessary and sufficient conditions for $\phi$ to be periodic are that $\phi(0)=\phi(2 \pi)$ and $\phi^{\prime}(0)=\phi^{\prime}(2 \pi)$. These conditions read

$$
M\left(\begin{array}{c}
a \\
b
\end{array}\right)=\left(\begin{array}{c}
-\phi_{p}(2 \pi) \\
-\phi_{p}^{\prime}(2 \pi)
\end{array}\right) \text { with } M=\left(\begin{array}{cc}
\phi_{1}(2 \pi)-1 & \phi_{2}(2 \pi) \\
\phi_{1}^{\prime}(2 \pi) & \phi_{2}^{\prime}(2 \pi)-1
\end{array}\right)
$$


This system has a single solution if and only if the determinant of $M$ is nonzero, which gives the dispersion relation

$$
D(c+i \epsilon) \equiv\left[\phi_{1}(2 \pi)-1\right]\left[\phi_{2}^{\prime}(2 \pi)-1\right]-\phi_{1}^{\prime}(2 \pi) \phi_{2}(2 \pi)=0 .
$$

The existence of modes (nontrivial solutions to (13)) is then equivalent to the zero values of the dispersion relations. When no mode exist, $D$ is nonzero and (12) has thus a unique periodic solution $\phi_{f}$ (14), with $a$ and $b$ the unique solution to (15).

Turning back to the inhomogeneous Rayleigh equation, the preceding discussion applies as soon as $\epsilon \neq 0$. We assume that no unstable mode exists, then $D(c+i \epsilon)$ is nonzero. Then the inhomogeneous Rayleigh equation has a unique solution for any $c+i \epsilon$, for nonzero $\epsilon$.

The limit $\epsilon \rightarrow 0$ of $\phi_{f}(c+i \epsilon)$ is nontrivial due to the existence of critical layers $y_{c}(c)$, for which the Rayleigh equation becomes singular. We study this limit in the following sections.

\subsubsection{Limit $\epsilon \rightarrow 0^{+}$for isolated critical layers}

We consider fixed values of $c$ which are on the range of $U: \min _{y}\{U(y)\}<c<\max _{y}\{U(y)\}$. In such a case, for any value of $c$, there exist one or several points $y_{l}$ such that $U\left(y_{l}\right)=c$. The inhomogeneous Rayleigh equation is then singular at such critical layers. We discuss in this section the case $U^{\prime}\left(y_{l}\right) \neq 0$ (isolated critical layers). The case $U^{\prime}\left(y_{l}\right)=0$ will be treated in the next section.

In order to properly study the limit $\epsilon \rightarrow 0$, we first build a solution to the homogeneous equation (10), which is regular at one of the critical layers $y=y_{l}$. We define $\phi_{r}(y, c)$ as the solution to (10) with $\phi_{r}\left(y_{l}, c\right)=0$ and $\phi_{r}^{\prime}\left(y_{l}, c\right)=1$. From (10), we have $\phi_{r}^{\prime \prime}\left(y_{l}, c\right)=U^{\prime \prime}\left(y_{l}\right) / U^{\prime}\left(y_{c}\right)$. We then have the expansion

$$
\phi_{r}(y, c)=\left(y-y_{l}(c)\right)\left[1+\frac{U^{\prime \prime}\left(y_{l}(c)\right)}{2 U^{\prime}\left(y_{l}(c)\right)}\left(y-y_{l}(c)\right)+o\left(y-y_{l}\right)\right]
$$

It can be shown that the solution $\phi_{r}(y, c)$ is an analytic function of $y$ in the vicinity of $y_{l}$, if we suppose that $U(y)$ is analytic in a vicinity of $y_{l}$. Moreover, from the definition $U\left(y_{l}(c)\right)=c$, because $U^{\prime}\left(y_{l}\right) \neq 0$, then $y_{l}(c)$ is analytic in a vicinity of $c$ and $d y_{l} / d c=1 / U^{\prime}\left(y_{l}\right)$. The solution $\phi_{r}\left(y, c^{\prime}\right)$ has then an analytic continuation for complex $c^{\prime}$ in the vicinity of $c$.

A classical result of the theory of differential equation of second order is that, if we already know a solution $\phi_{r}$, all other solutions $\phi$ are expressed in terms of $\phi_{r}$ by quadratures. The recipe for this is to look for solutions under the form $\phi=u \phi_{r}$, look for the equation verified by $u$ and integrate it. We apply this recipe to the inhomogeneous Rayleigh equation (9). Then any solution $\phi$ to (9) is expressed as

$$
\begin{gathered}
\phi(y)=d \phi_{r}(y)+\phi_{r}(y) \int_{y_{0}}^{y} \frac{(e+f)}{\phi_{r}^{2}} \text { with } \\
f(y)=\int_{y_{0}}^{y} d y_{2} \frac{\omega\left(y_{2}, 0\right) \phi_{r}\left(y_{2}\right)}{i k\left(U\left(y_{2}\right)-c-i \epsilon\right)},
\end{gathered}
$$

and where $d$ and $e$ are constants.

We study the behavior of the previous expression close to $y_{l}$. We first note that $f$ is analytic close to $y_{l}$. Then using the expansion (17), we conclude that

$$
\phi(y, c+i \epsilon)=d \phi_{r}(y, c+i \epsilon)+g \phi_{r}(y, c+i \epsilon) \log \left(y-y_{l}(c+i \epsilon)\right)+e \phi_{g}(y, c+i \epsilon)+\phi_{h}(y, c+i \epsilon)
$$

where $\phi_{g}$ and $\phi_{h}$ are analytic functions of $y$ close to $y_{l}$, and where $g$ is a constant that depends on $f\left(y_{l}\right), f^{\prime}\left(y_{l}\right)$ and $e$.

The interpretation of (19) depends on which determination of the logarithm we use. Using $d y_{l} / d c=1 / U^{\prime}\left(y_{l}\right)$ (discussed above), we have $y_{l}(c+i \epsilon)=y_{l}(c)+i \epsilon / U^{\prime}\left(y_{l}\right)+o(\epsilon)$. We choose a determination of the logarithm such that $\log \left(y-y_{l}(c)-i \epsilon / U^{\prime}\left(y_{l}\right)\right)$ remains analytic for positive $\epsilon$. Then the study of the limit $\epsilon \rightarrow 0$ of equation (14) is easily done, we denote this limit $\phi(y, c+i 0)$. Using that $\phi_{r}$ and $\phi_{g}$ depends analytically on $c$, we obtain

$$
\phi(y, c+i 0)=d \phi_{r}(y, c)+g \phi_{r}(y, c) \log \left|y-y_{l}\right|+e \phi_{g}(y, c)+\phi_{h}(y, c) \text { for } y>y_{l} \text { and }
$$




$$
\phi(y, c+i 0)=\left(d-i \pi \operatorname{sgn}\left(U^{\prime}\left(y_{l}\right)\right) g\right) \phi_{r}(y, c)+g \phi_{r}(y, c) \log \left|y-y_{l}\right|+e \phi_{g}(y, c)+\phi_{h}(y, c) \text { for } y<y_{l}
$$

where $\operatorname{sgn}\left(U^{\prime}\left(y_{l}\right)\right)$ is the sign of $U^{\prime}\left(y_{l}\right)$.

From this, we conclude that, for given $d$ and $e$, the solution to the inhomogeneous Rayleigh equation (9) converges, for $\epsilon \rightarrow 0$, towards a function $\phi(y, c+i 0)$, which is an analytical function of $y$, except for $y=y_{l}$ where it has a logarithmic singularity. It is continuous at $y=y_{l}$. This result is valid close to any single critical layer $y_{l}$. If two or several critical layer $y_{l, i}$ exist in the interval $y \in(0,2 \pi)$, then the result is easily extended, and holds with a singularity at each critical layer.

Is this result also true for the solution $\phi_{\omega}(y, c+i \epsilon)$ of the inhomogeneous Rayleigh equation with boundary conditions? In order to answer this, we now turn again to the construction of section 2.3.1 The result of the previous paragraph is applied alternatively to $\phi_{1}(y, c+i \epsilon), \phi_{2}(y, c+i \epsilon)$ and to $\phi_{p}(y, c+i \epsilon)$. We thus conclude that all these three functions have well-defined limits for $\epsilon \rightarrow 0^{+}$, and that these limits are continuous functions of $y$, which have logarithmic singularities in their derivative for each critical layer. We can then extent the definition of the dispersion relation to $\epsilon \rightarrow 0^{+}$with $D_{+}(c)=\lim _{\epsilon \rightarrow 0^{+}} D(c+i \epsilon)$. $D_{+}(c)$ verifies (16) for which we have proved that all terms have a finite limit when $\epsilon \rightarrow 0^{+}$. Then we conclude that the two parameters $a$ and $b$ in equation (14) have finite limits when $\epsilon \rightarrow 0^{+}$. These limit values are given by equation (15), where each term has a finite limit.

We thus conclude that the solution to the Rayleigh equation with boundary conditions $\phi_{\omega}(y, c+i \epsilon)$ has a finite limit $\phi_{+}(y, c)$ for $\epsilon \rightarrow 0^{+}$. Moreover, $\phi_{+}(y, c)$ is a continuous function of $y$ that has a logarithmic singularity at each critical layer, giving a finite jump for the first derivative.

Let us denote $\Delta \phi_{+}$this jump. From the previous analysis we know that

$$
\phi_{+}(y, c)=a+b\left(y-y_{l}\right)+\Delta \phi_{+}\left(y-y_{l}\right) \log \left|y-y_{l}\right|+\mathcal{O}\left(y-y_{l}\right)^{2}
$$

Using this expansion, a direct analysis of the dominant term in (9) leads to

$$
\Delta \phi_{+}=\frac{\omega\left(y_{l}, 0\right)+i k U^{\prime \prime}\left(y_{l}\right) \phi_{+}\left(y_{l}, c\right)}{i k U^{\prime}\left(y_{l}\right)} .
$$

The jump in the derivative thus depends on the value of $\phi_{+}$which is a non local quantity $\left(\phi_{+}\right.$depends on the whole profile $U$ ).

Because $\phi_{r}$ and $y_{l}$ are analytic functions of $c$, the construction of $\phi_{+}$can be extended analytically when $c$ is varied. Then from (22), using $y_{l}\left(c^{\prime}\right)=y_{l}(c)+\left(c^{\prime}-c\right) / U^{\prime}\left(y_{l}\right)$, one sees that, for fixed $y$ :

$$
\phi_{+}(y, c)=\Delta \phi_{c}(U(y)-c) \log (U(y)-c)+\phi_{a}(y, c)
$$

where $\phi_{a}(y, c)$ is analytic close to $c=U(y)$ and with

$$
\Delta \phi_{c}=\frac{\Delta \phi_{+}}{U^{\prime}(y)}=\frac{\omega(y, 0)+i k U^{\prime \prime}(y) \phi_{+}(y, U(y))}{i k\left(U^{\prime}(y)\right)^{2}} .
$$

We illustrate the preceding results with numerical solutions of the inhomogeneous Rayleigh equations on a doubly periodic domain, and the base flow $U(y)=\cos y$ (the Kolmogorov flow).

We follow the algorithm described in section 2.3.1, that is, computing $\phi_{1}, \phi_{2}$, and $\phi_{p}$, and then by using them, computing the solution to the inhomogeneous Rayleigh equation for $c^{\prime}=c+i \epsilon$ for small but nonzero value of $\epsilon$. In order to numerically compute the solution to the differential equations (for $\phi_{1}, \phi_{2}$, and $\phi_{p}$ ), we use an adaptive method to deal with the singularity close to the critical layers. An extreme precision is required in order to obtain satisfactory results.

In order to test the quality of the numerical simulations, we compute the Wronskian $W=\phi_{1}(y) \phi_{2}^{\prime}(y)-$ $\phi_{2}(y) \phi_{1}^{\prime}(y)$. From the general theory of differential equations of second order, we know that $W$ does not depend on $y$. Here, from the values of $\phi_{1}$ and $\phi_{2}$ at $y=0$, given by their definition, we have $W=1$. We test the accuracy of this in all our numerical simulations. For instance in the case of simple critical layers, using the Matlab function ode 45 , and fixing the relative error and the absolute error parameters of this function to $10^{-13}$, we obtain solutions for which errors in $W$ are typically smaller than $10^{-6}$ for $\epsilon=10^{-4}$. 


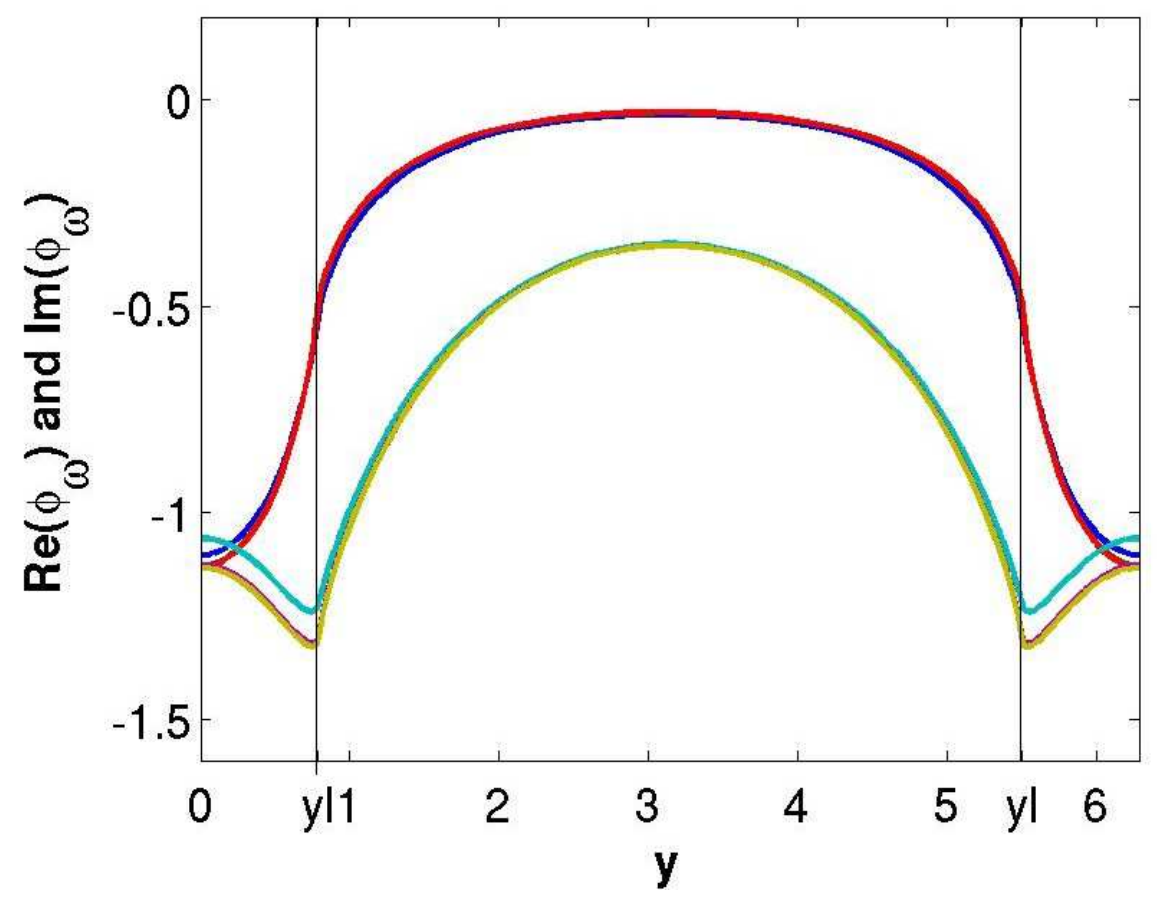

Figure 2: The real ( $\Re$, upper set of curves) and the imaginary part ( $\Im$, lower set of curves) for the solution $\phi_{\omega}(y, c+i \epsilon)$ to the inhomogeneous Rayleigh equation (9), in the case $\omega(y, 0)=1, c=\sqrt{2} / 2\left(y_{l}=\pi / 4\right.$ and $\left.y_{l}=7 \pi / 4\right), k=1.5$. The different curves show the results for $\epsilon=10^{-2}$ (blue $(\Re)$ and light blue $\left.(\Im)\right), \epsilon=10^{-3}$ (green $(\Re)$ and magenta $(\Im))$ and $\epsilon=10^{-4}\left(\right.$ red $(\Re)$ and yellow $(\Im)$ ). The curves for $\epsilon=10^{-3}$ and $\epsilon=10^{-4}$ are nearly indistinguishable, showing good convergence. 


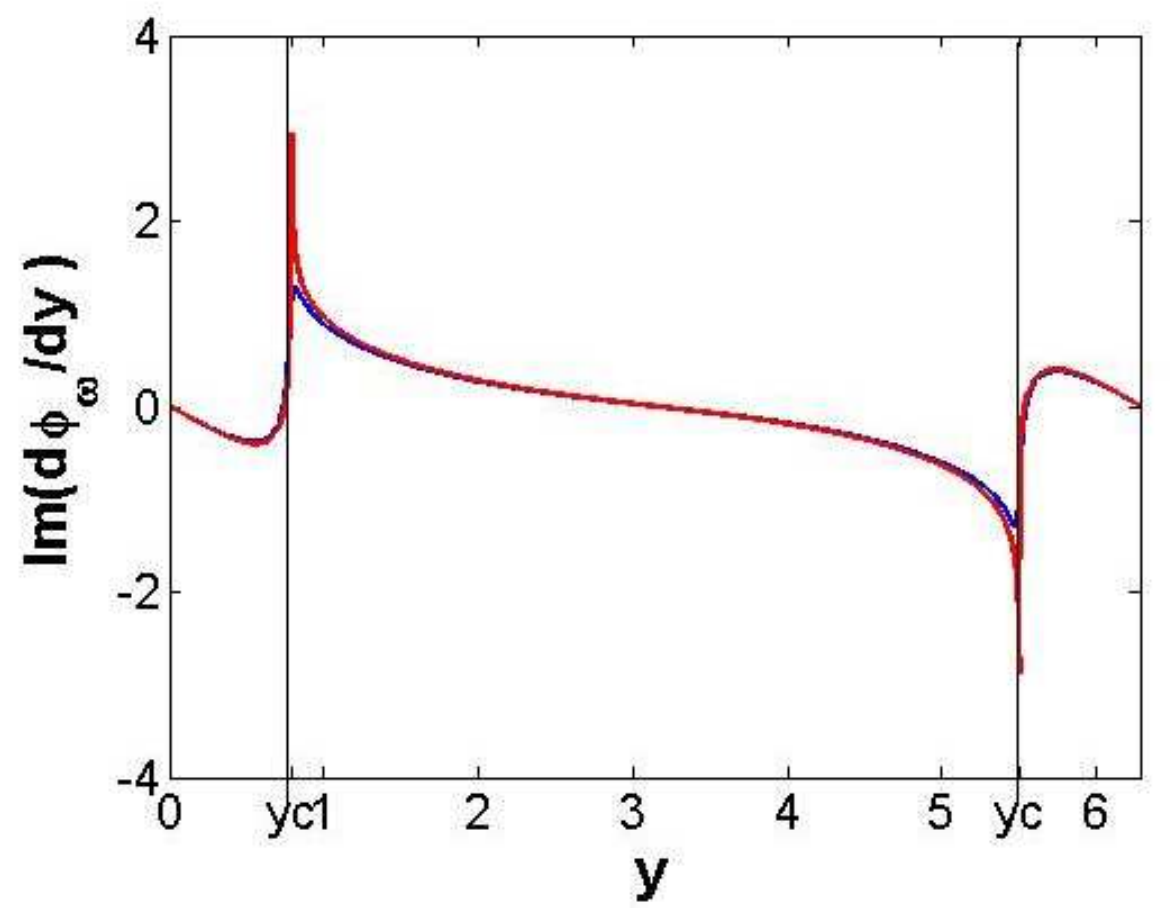

Figure 3: Same as the previous figure, but for the imaginary part of the derivative $\phi_{\omega}^{\prime}(y, c+i \epsilon)$ of the solution to the inhomogeneous Rayleigh equation (9) (blue for $\epsilon=10^{-2}$, green for $\epsilon=10^{-3}$ ) and red for $\epsilon=10^{-4}$. The green and red curves are nearly indistinguishable.

Figure 2 shows the real and the imaginary part for the solution $\phi_{\omega}(y, c+i \epsilon)$ to the inhomogeneous Rayleigh equation (9), in the case $\omega(y, 0)=1, c=\sqrt{2} / 2\left(y_{l}=\pi / 4\right.$ and $\left.y_{l}=7 \pi / 4\right), k=1.5$ for the values $\epsilon=10^{-2}$, $\epsilon=10^{-3}$ and $\epsilon=10^{-4}$. This illustrates the convergence of the solutions $\phi_{\omega}(y, c+i \epsilon)$ to a continuous function $\phi_{\omega}(y, c+i 0)$. The visible kinks close to the critical layers suggest the discontinuity of the derivative. This is actually verified and illustrated in figure 3 , that shows the derivative with respect to $y, \phi_{\omega}^{\prime}(y, c+i \epsilon)$.

\subsubsection{Limit $\epsilon \rightarrow 0^{+}$for critical layer of stationary streamlines}

We now consider the case of a critical layer that correspond to a stationary streamline $\left(y_{l}=y_{0}\right.$, where $U\left(y_{0}\right)=c_{0}$, $U^{\prime}\left(y_{0}\right)=0$ and $\left.U^{\prime \prime}\left(y_{0}\right) \neq 0\right)$.

In order to properly study the limit $\epsilon \rightarrow 0$ in this case, we first build a solution to the homogeneous equation (10), which is regular at the critical layer $y=y_{0}$. We define $\phi_{r}\left(y, c_{0}\right)$ as the solution to (10) with $\phi_{r}\left(y_{0}, c_{0}\right)=0$, $\phi_{r}^{\prime}\left(y_{0}, c_{0}\right)=0$ and $\phi_{r}^{\prime \prime}\left(y_{0}, c_{0}\right)=1$. Such a solution can be shown to exist by a series expansion in powers of $\left(y-y_{0}\right)$. It can be shown that $\phi_{r}\left(y, c_{0}\right)$ is an analytic function of $y$ in the vicinity of $y_{0}$, if we suppose that $U(y)$ is analytic in the vicinity of $y_{0}$.

However, by contrast to the case of isolated critical layers analyzed in section (2.3.2), the solution $\phi_{r}\left(y, c_{0}\right)$ is not analytic in the vicinity of $c_{0}$. Then the approach of the previous section can not be generalized.

Let us first prove that it exists a solution to the inhomogeneous Rayleigh equation, for $\epsilon=0$, which is continuously differentiable at $y_{0}$. We start from expression (18). $f$ is analytic in $y_{0}$. Let us choose $e=-f\left(y_{0}\right)$ and $b=0$. Then we obtain the particular solution

$$
\phi_{\omega, 0}(y)=\phi_{r}(y) \int_{y_{0}}^{y} \frac{\left(f-f\left(y_{0}\right)\right)}{\phi_{r}^{2}} .
$$

Noting that the expansion of $\phi_{r}$ begins at order 2 in $\left(y-y_{0}\right)$, we easily prove that

$$
\phi_{\omega, 0}(y)=\phi_{i}(y)+g \phi_{r}(y) \log \left|y-y_{0}\right|
$$


where $g$ is a constant and $\phi_{i}$ is an analytic function of $y$. We note that $\phi_{\omega, 0}(y)$ is continuously differentiable at the critical layer $y_{0}$. This solution is defined locally, in an interval where $\phi_{r}$ has no other zero than $y_{0}$. However, it can be extended to the whole interval $y \in(0,2 \pi)$, because equation (9) is not singular in other points than $y_{0}$.

By contrast to the situation obtained for isolated critical points, we can not make an analytical continuation of the solution (25) for complex $c_{0}+i \epsilon$. We thus follow another route.

We note that we can add $b \phi_{i}$ to $\phi_{\omega, 0}(y)$, where two different values for $b$ can be chosen for $y<y_{0}$ and for $y>y_{0}$. The function

$$
\left\{\begin{array}{l}
\phi_{+}\left(y, c_{0}\right)=b^{-} \phi_{r}(y)+\phi_{\omega, 0}(y) \text { for } y<y_{0} \\
\phi_{+}\left(y, c_{0}\right)=b^{+} \phi_{r}(y)+\phi_{\omega, 0}(y) \text { for } y>y_{0}
\end{array}\right.
$$

is actually a solution to the inhomogeneous Rayleigh equation (9) for any $y \neq y_{0}$ which is continuously differentiable in $y_{l}$. It is thus a solution to (9).

The values of $b^{+}$and $b^{-}$can be chosen in order to satisfy the boundary conditions. For instance, for $2 \pi$-periodic solutions, the boundary conditions are equivalent to

$$
N\left(\begin{array}{c}
b^{-} \\
b^{+}
\end{array}\right)=\left(\begin{array}{c}
\phi_{\omega, 0}(2 \pi)-\phi_{\omega, 0}(0) \\
\phi_{\omega, 0}^{\prime}(2 \pi)-\phi_{\omega, 0}^{\prime}(0)
\end{array}\right) \text { with } N=\left(\begin{array}{cc}
\phi_{r}(0) & -\phi_{r}(2 \pi) \\
\phi_{r}^{\prime}(0) & -\phi_{r}^{\prime}(2 \pi)
\end{array}\right)
$$

The determinant of $N$ then plays the role of a dispersion relation for neutral mode associated to the stationary streamlines. It reads

$$
D_{s}=-\phi_{r}(0) \phi_{r}^{\prime}(2 \pi)+\phi_{r}(2 \pi) \phi_{r}^{\prime}(0)
$$

When no such mode exists, equation (28) is solved, and we obtain a solution to the inhomogeneous Rayleigh equation that verifies the boundary conditions.

We have thus constructed a solution to the inhomogeneous Rayleigh equation for real $c_{0}=U\left(y_{0}\right)$, where $y_{0}$ is a stationary point of $U$ such that $U^{\prime \prime}\left(y_{0}\right) \neq 0$.

We illustrate the preceding results with numerical solutions of the inhomogeneous Rayleigh equations on a doubly periodic domain, for the Kolmogorov base flow $U(y)=\cos y$.

The numerical computation follows the same rules are the one described in section 2.3.2. We note that using the Matlab function ode45, and fixing the relative error and the absolute error parameters of this function to $10^{-13}$, we obtain solutions for which errors in $W$ are typically smaller than $10^{-2}$ for $\epsilon=10^{-3}$. It is thus much harder to obtain good quality numerical simulation in that case, than in the case of isolated critical layers discussed in section 2.3.2.

Figure 4 shows the real and the imaginary part for the solution $\phi_{\omega}\left(y, c_{0}+i \epsilon\right)$ to the inhomogeneous Rayleigh equation (9), in the case $\omega(y, 0)=1$; with a critical layer corresponding to a stationary point $\left(c_{0}=1, y_{0}=0\right)$ and with $k=1.5$; for the values $\epsilon=10^{-2}, \epsilon=5.10^{-3}$ and $\epsilon=10^{-3}$. This illustrates the convergence of the solutions $\phi_{\omega}\left(y, c_{0}+i \epsilon\right)$ to a continuous function $\phi_{+}\left(y, c_{0}\right)$. It turns out that the real part converges to zero. The same results are also presented for the derivative with respect to $y, \phi_{\omega}^{\prime}\left(y, c_{0}+i \epsilon\right)$, on figure 5.

We now turn to the derivation of a property of such solutions, that will be very important in the discussion of the asymptotic behavior of the linearized 2D Euler equations. We have shown that $\phi_{+}\left(y, c_{0}\right)$ is continuously differentiable at $y_{l}$, and has a second order logarithmic singularity at $y_{0}$ (see (26) and (27)). Then a direct inspection of the leading singularity in equation (9), of order $\left(y-y_{c}\right)^{-1}$, leads to the conclusion that

$$
i k U^{\prime \prime}\left(y_{0}\right) \phi_{+}\left(y_{0}, U\left(y_{0}\right)\right)+\omega\left(y_{0}, 0\right)=0
$$

\subsubsection{The asymptotic vorticity field}

Using the results of the previous section, we prove in this section that the vorticity field converges, for large time, towards a field oscillating at a multiple of the streamline frequency. More precisely, we prove that

$$
\omega(y, t) \underset{t \rightarrow \infty}{\sim} \omega_{\infty}(y) \exp (-i k U(y) t)+\mathcal{O}\left(\frac{1}{t^{\gamma}}\right)
$$

In particular, for any stationary point $y_{0}, \omega_{\infty}\left(y_{0}\right)=0$. This essential property means that the vorticity cancels rapidly at any stationary streamline. This is the mechanism of vorticity depletion at the stationary streamlines, 


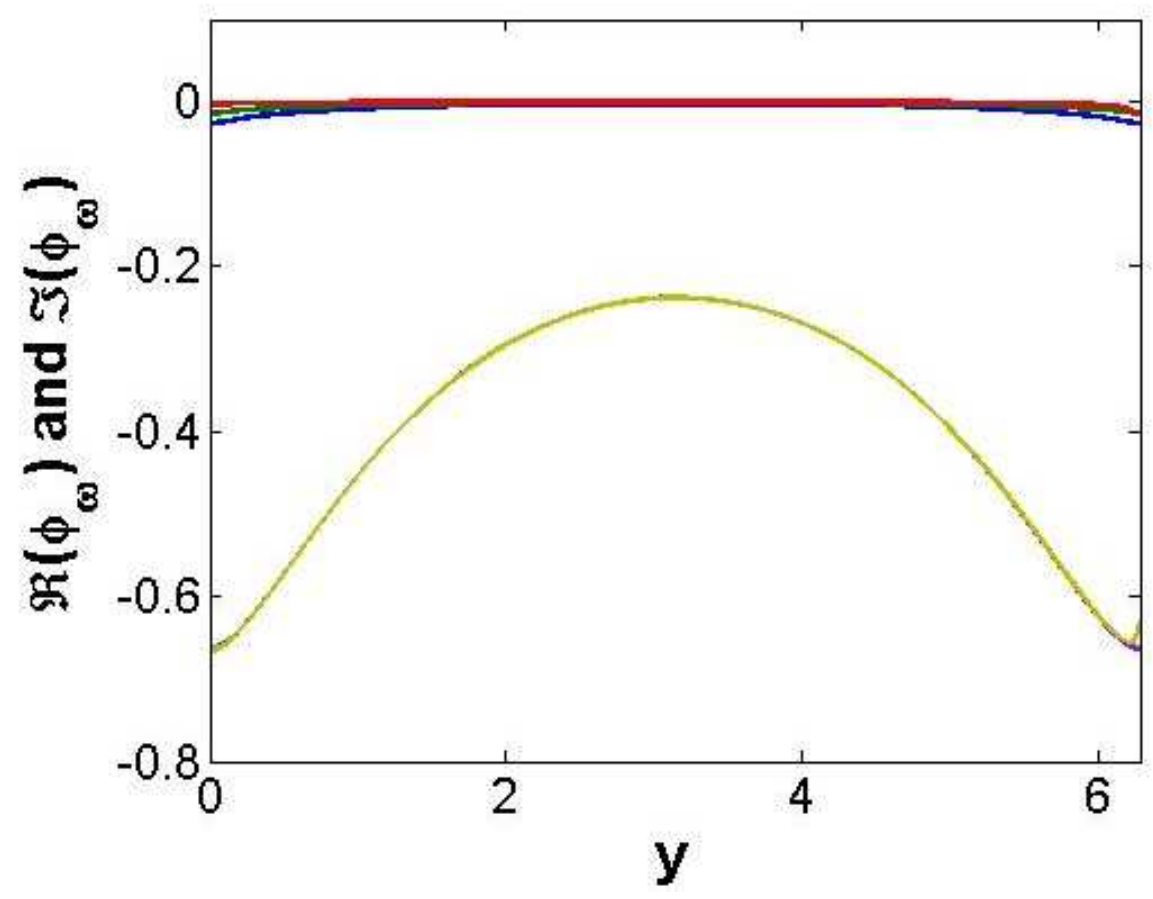

Figure 4: The real ( $\Re$ ) and the imaginary $(\Im)$ part for the solution $\phi_{\omega}\left(y, c_{0}+i \epsilon\right)$ to the inhomogeneous Rayleigh equation (9), in the case $\omega(y, 0)=1$; with a critical layer corresponding to a stationary point $\left(c_{0}=1, y_{l}=0\right)$ and with $k=1.5$. The different curves show the results for $\epsilon=10^{-2}$ (blue $(\Re)$ and light blue $(\Im)$ ), $\epsilon=5.10^{-3}$ (green $(\Re)$ and magenta $(\Im)$ ) and $\epsilon=10^{-3}\left(\right.$ red $(\Re)$ and yellow $(\Im)$ ). The curves for $\epsilon=5.10^{-3}$ and $\epsilon=10^{-3}$ are indistinguishable, showing good convergence. The real part is the ensemble of curves that converge to zero.

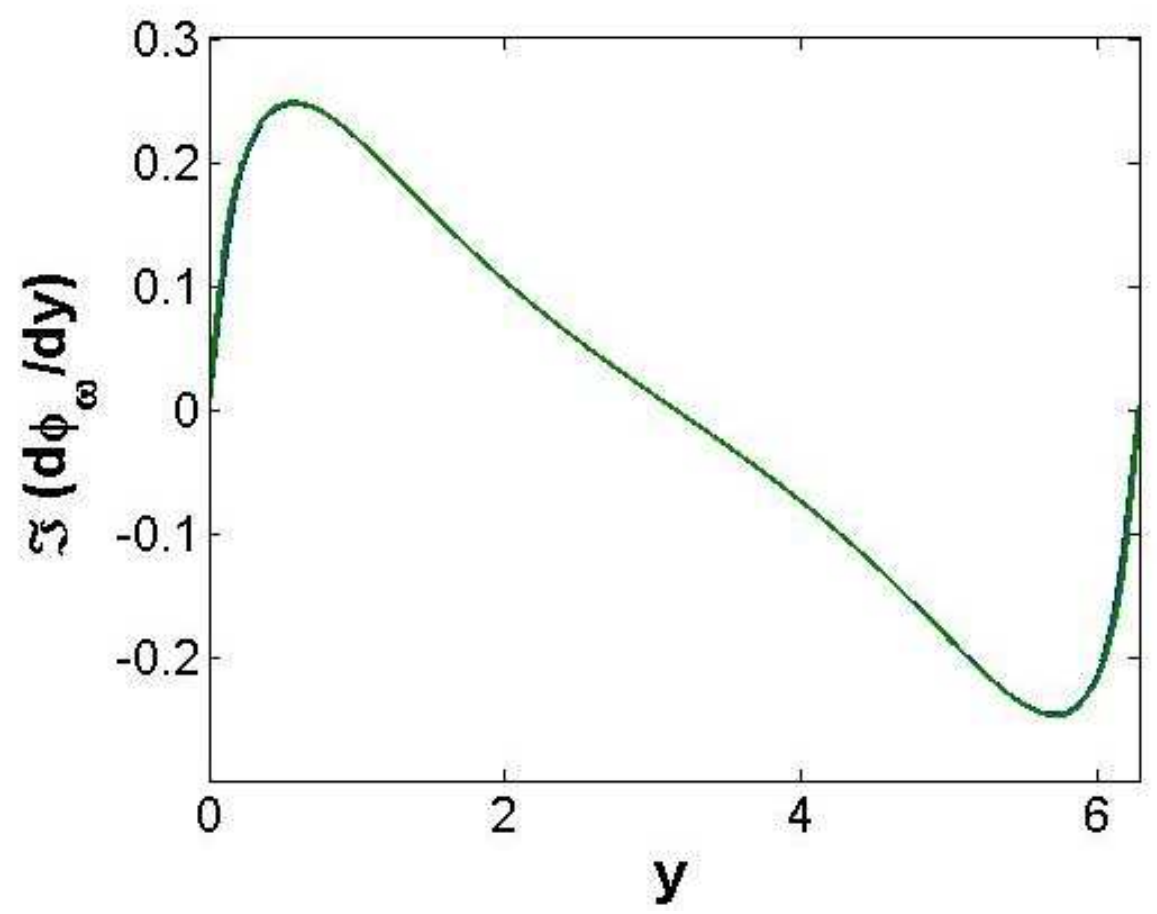

Figure 5: Same as the previous figure, but for the imaginary part of the derivative $\phi_{\omega}^{\prime}\left(y, c_{0}+i \epsilon\right)$ of the solution to the inhomogeneous Rayleigh equation (9). All three curves are indistinguishable. 
discussed in the introduction.

Using (11) and (8) we have

$$
\hat{\omega}(y, c+i \epsilon) \underset{\epsilon \rightarrow 0^{+}}{\sim} \frac{i k U^{\prime \prime}(y) \phi_{+}(y, c)+\omega(y, 0)}{i k\left(U(y)-c-i 0^{+}\right)}
$$

Thanks to the analysis of the properties of $\phi$ in the previous section, we know that all its singularities are integrable. We thus see that for any $c, \hat{\omega}(., c)$ has non integrable singularities at each critical layer $y_{l}$. For fixed $y, \hat{\omega}(y,$.$) has a single singularity for the velocity c=U(y)$. Using (32), we write the inverse Laplace transform (77) on the Bromwich contour defined by $p=-i k(c+i \epsilon)$ with $\epsilon>0$ and $-\infty \leq c \leq+\infty$.

$$
\omega(y, t)=\frac{1}{2 \pi i} \int_{-\infty}^{+\infty} d c \frac{\exp (-i k(c+i \epsilon) t)}{U(y)-c-i \epsilon}\left[i k U^{\prime \prime}(y) \phi(y, c+i \epsilon)+\omega(0, y)\right]
$$

We first estimate the contribution of the pole

$\frac{1}{2 \pi i} \int_{-\infty}^{+\infty} d c \frac{\exp (-i k(c+i \epsilon) t)}{U(y)-c-i \epsilon}\left[i k U^{\prime \prime}(y) \phi_{+}(y, U(y))+\omega(0, y)\right]=\left[i k U^{\prime \prime}(y) \phi_{+}(y, U(y))+\omega(y, 0)\right] \exp (-i k U(y) t)$

using the standard deformation of the contour of the complex integral, and the residue theorem. The remainder contribution to the vorticity (33) is then

$$
\frac{k U^{\prime \prime}(y)}{2 \pi} \int_{-\infty}^{+\infty} d c \frac{\phi_{+}(y, c)-\phi_{+}(y, U(y))}{U(y)-c} \exp (-i k c t) .
$$

This integral is an oscillating integral. For large times, it thus gives a contribution of order $\mathcal{O}\left(1 / t^{\gamma}\right)$ where $\gamma$ depends on the order differentiability of $\phi_{+}(y, c)$ as a function of $c$. The result (31) is thus proved, and we have

$$
\omega_{\infty}(y)=i k U^{\prime \prime}(y) \phi_{+}(y, U(y))+\omega(y, 0) .
$$

We remark that for any point $y_{1}$ where $U^{\prime \prime}\left(y_{1}\right)$ vanishes, $\omega_{\infty}\left(y_{1}\right)=\omega\left(y_{1}, 0\right)$. This could have been anticipated as for such points $y_{1}$, from (6), we trivially have $\omega\left(y_{1}, t\right)=\omega\left(y_{1}, 0\right) \exp \left(-i k U\left(y_{1}\right) t\right)$ for any time $t$.

Using (30) and (34), we deduce that

$$
\omega_{\infty}\left(y_{0}\right)=0
$$

This result means that the vorticity tends to zero for large time for any stationary streamlines $y_{0}$. This vorticity depletion at the stationary streamline is, from a mathematical point of view, a nontrivial consequence of the Laplace transform analysis, and of the regularization of the resolvent operator at stationary velocities. As will be illustrated in section 4, using numerical simulation, it is a striking dynamical effect leading to the disappearance of any filament in the area close to the critical layer of a stationary point of the profile $U$. This has a large qualitative impact on the flow structure and evolution.

This effect comes from the term $v_{y} U^{\prime \prime}(y)$ in the linearized Euler equation (6) ; it is thus a consequence of the effect of the transverse velocity on the background vorticity. Because $v_{y}$ is a non local quantity, depending on the evolution of the vorticity field everywhere in the domain, this effect is a non local, non trivial one that we are not able to explain easily heuristically.

Besides these theoretical results, the Laplace tools are very interesting as they allow the computation of asymptotic behavior of the flow without relying on a complex direct numerical computation. Moreover, whereas in asymptotic approaches like the Lundgren's one, where the asymptotic profile $\omega_{\infty}(y)$ is not determined, here we can compute it from (34).

Using this last procedure and the numerical computations of the resolvent $\phi_{\omega}$, described in sections 2.3 .2 and 2.3.3, we compute the asymptotic vorticity profiles. They are represented in figures [10, 11] and 16]; and discussed in more details in section 4.2 . 


\subsubsection{The asymptotic velocity field}

In this section we study the asymptotic behavior of the velocity field. We prove that the velocity field decays algebraically for large times:

$$
\begin{aligned}
& v_{x}(y, t) \underset{t \rightarrow \infty}{\sim} \frac{\omega_{\infty}(y)}{i k U^{\prime}(y)} \frac{\exp (-i k U(y) t)}{t} \text { and } \\
& v_{y}(y, t) \underset{t \rightarrow \infty}{\sim} \frac{\omega_{\infty}(y)}{i k\left(U^{\prime}(y)\right)^{2}} \frac{\exp (-i k U(y) t)}{t^{2}} ;
\end{aligned}
$$

where $\omega_{\infty}$ is the asymptotic vorticity profile (31134).

We first explain this result starting from the asymptotic vorticity derived in the previous section (31), and using large time asymptotic behavior of oscillating integrals. This argument is heuristically very interesting. However it will be valid only when the contributions of stationary points $y_{0}$ are negligible, and when the convergence of the vorticity towards the asymptotic vorticity is sufficiently rapid. Indeed, the convergence towards the Lundgren's ansatz has to be uniformly more rapid than the derived algebraic laws. This last point is actually true only for strictly monotonic velocity profiles $U$ as will be discussed below.

In order to give a proof of the results (35) and (35) valid also for profile $U$ with stationary streamlines, we give a more general argument based on Laplace transform at the end of this section.

Oscillating integrals We begin with the expression of the velocity from the vorticity field using a Green function formalism. We have

$$
\mathbf{v}(y, t)=\int d y^{\prime} \mathbf{G}_{k}\left(y, y^{\prime}\right) \omega\left(y^{\prime}, t\right)
$$

where $\mathbf{G}_{k}$ is defined from (5):

$$
\mathbf{G}_{k}\left(y, y^{\prime}\right)=\left(-\frac{\partial H_{k}}{\partial y}, i k H_{k}\right)\left(y, y^{\prime}\right) \text { with } \frac{\partial^{2} H_{k}}{\partial y^{2}}-k^{2} H_{k}=\delta\left(y-y^{\prime}\right),
$$

with the periodic boundary conditions on $y$. Using the asymptotic result on the vorticity field, we thus have

$$
\mathbf{v}(y, t) \underset{t \rightarrow \infty}{\sim} \int d y^{\prime} \mathbf{G}_{k}\left(y, y^{\prime}\right) \omega_{\infty}\left(y^{\prime}\right) \exp (-i k U(y) t) .
$$

We consider the asymptotic behavior, for large times $t$, of the oscillating integral (38). Since Kelvin, very classical results do exist for the asymptotic behavior of such integrals, the most famous result being the stationary phase approximation. Such results are discussed in appendix A.

An essential point, which makes this case different from the more classical ones, is that the Green function $\mathbf{G}_{k}\left(y, y^{\prime}\right)$ is not smooth everywhere: it is smooth except for the singularity when $y=y^{\prime}$. We prove in appendix A that if $U(y)$ has no stationary points (for all $y, U^{\prime}(y) \neq 0$ ), then results (35) [36) are valid, the main contribution being related to the singularities of the Green function.

If the velocity field $U(y)$ has $M$ stationary points $y_{m}\left(U^{\prime}\left(y_{m}\right)=0\right)$, then the contributions of the stationary points, generically of order $1 / \sqrt{t}$, usually dominate the contributions of the singularity of the Green function $\mathbf{G}_{k}$, in integrals like (38). If $\omega_{\infty}\left(y_{m}\right) \neq 0$, the classical stationary phase approximation (see appendix A) would lead to

$$
\mathbf{v}(y, t) \underset{t \rightarrow \infty}{\sim} \sum_{m=1 . . M} \mathbf{G}_{k}\left(y, y_{m}\right) \omega_{\infty}\left(y_{m}\right) \sqrt{\frac{2 \pi}{\left|k U^{\prime \prime}\left(y_{m}\right)\right|}} \exp \left(\frac{i \epsilon_{m} \pi}{4}\right) \frac{\exp \left(-i k U\left(y_{m}\right) t\right)}{\sqrt{t}},
$$

where $\epsilon_{m}$ is the sign of $-k U^{\prime \prime}\left(y_{m}\right)$.

However, a remarkable fact is that for any stationary point $y_{0}$ (such that $U^{\prime}\left(y_{0}\right)=0$ ), due to the vorticity depletion mechanism discussed in section 2.3.4 and proved in section 2.3.3, $\omega_{\infty}\left(y_{0}\right)=0$. Then the leading order contribution from the stationary phase approximation vanishes. The analysis could proceed in order to determine the next leading order term in the expansion, from (38), expected to be of order $1 / t^{3 / 2}$. However, such a detailed analysis is useless, because the convergence of $\omega(., t)$ towards the asymptotic vorticity profile $\omega_{\infty}$ is too slow, in the vicinity of a stationary streamline. Actually the error due to the slow convergence towards the Lundgren's profile gives contributions which are also of order $1 / t^{3 / 2}$. This will be illustrated using direct numerical simulations in section 4 (see figure 9 page 24, figure 15 page 40 and the related text). 
Laplace tools In order to give a precise argument for the results (35), we use Laplace tools. We first note that $v_{y}=i k \psi$, and study the asymptotics for the stream function $\psi$. Starting from the inverse Laplace transform of $\psi$, we have

$$
\psi(y, t)=\frac{k}{2 \pi} \int_{-\infty}^{+\infty} d c \phi_{+}(y, c) \exp (-i k c t)
$$

where $\phi_{+}(y,$.$) is the limit of \phi(y, c+i \epsilon)$ for $\epsilon \rightarrow 0$. (39) is an oscillating integral. We use that for any $y$ for which $U^{\prime}(y) \neq 0, \phi_{+}(y, c)$ is twice differentiable except at $c=U(y)$ where it has a logarithmic singularity $\Delta \phi_{c}(c-U(y)) \log (c-U(y))$ (see equation (23)). Then the large time asymptotics of $\psi$ is due to this singularity. In order to evaluate it, we part integrate twice (39) and evaluate the contribution of the singularity with the residue theorem. Then the leading order contribution is obtained as,

$$
\psi(y, t) \underset{t \rightarrow \infty}{\sim} \frac{\omega_{\infty}(y)}{\left(i k U^{\prime}(y)\right)^{2}} \frac{\exp (-i k U(y) t)}{t^{2}},
$$

where we have used (24) and (34) in order to express $\Delta \phi_{c}$.

We note that (36) follows immediately from (40) and from the relation $v_{y}=i k \psi$. The asymptotic result (35) for the transverse velocity $v_{x}$ can be derived by following similar arguments as the one just described for $\psi$.

The above argument uses the explicit prediction (23) for the singularity of $\phi_{+}(y, c)$ as a function of $c$. The expressions (23) and (24) are valid only when $y$ is not a stationary streamline $\left(U^{\prime}(y) \neq 0\right)$. For stationary streamlines $y_{m}$ we have no theoretical predictions. Direct numerical computation in section 4.2 will lead us to conjecture that for such special points $\psi\left(y_{m}, t\right) \underset{t \rightarrow \infty}{\sim} C_{1} \exp \left(-i k U\left(y_{m}\right) t\right) / t^{3 / 2}, v_{x}\left(y_{m}, t\right) \underset{t \rightarrow \infty}{\sim} C_{2} \exp \left(-i k U\left(y_{m}\right) t\right) / t^{3 / 2}$ and $v_{y}\left(y_{m}, t\right) \underset{t \rightarrow \infty}{\sim} i k C_{1} \exp \left(-i k U\left(y_{m}\right) t\right) / t^{3 / 2}$. We note that this exponent $3 / 2$ is not related to a contribution from the stationary phase approximation.

We thus conclude that the results (35) and (36) are valid for monotonic profiles, but also for base flow with stationary streamlines. This is in marked contrast to what was thought in many previous publications based on the asymptotic expansions and the stationary phase arguments. This is mainly due to the vorticity depletion mechanism at the stationary streamlines discussed in the previous section. We also stress that, using Laplace tools, the asymptotic profile $v_{\infty}(y)$ can be numerically computed easily, without relying on direct numerical computations of the Euler equations.

We have theoretical predictions for the power law in the asymptotic behavior of the perturbation velocity, for all points of the domain except along the stationary streamlines.

\section{$3 \quad$ Asymptotic stability of parallel flows for the Euler equations}

In the previous section, we have obtained results for the asymptotic behavior of the linearized Euler equations, with initial conditions close to some parallel flows $\mathbf{v}_{0}(\mathbf{r})=U(y) \mathbf{e}_{x}$. We now address the evolution of the same initial conditions by the nonlinear Euler equation (3). The aim of this section is to explain why the linearized dynamics will be a good approximation for the dynamics for any time $t$, and to explain why the flow velocity is asymptotically stable (in kinetic energy norm), for small initial perturbation of the vorticity (in the enstrophy norm).

We consider the initial vorticity $\Omega(x, y, 0)=-U^{\prime}(y)+\epsilon \omega(x, y, 0)$, where $\epsilon$ is small. We suppose, without loss of generality that $\int d x \omega=0$. The perturbation $\omega$ can be decomposed in Fourier modes along the $x$ direction

$$
\omega(x, y, t)=\sum_{k} \omega_{k}(y, t) \exp (i k x)
$$

From the Euler equations (3), the equation for the evolution of $\omega_{k}$ reads

$$
\frac{\partial \omega_{k}}{\partial t}+i k U(y) \omega_{k}-i k \psi_{k} U^{\prime \prime}(y)=-\epsilon N L \text { with } N L=\sum_{l}\left\{-i k \frac{\partial \psi_{l}}{\partial y}(y, t) \omega_{k-l}(y, t)+\frac{\partial}{\partial y}\left[i l \psi_{l}(y, t) \omega_{k-l}(y, t)\right]\right\} .
$$

The left hand side is the linearized Euler equation, whereas the right hand side are the nonlinear corrections. We want to prove that, for sufficiently small $\epsilon$, neglecting the nonlinear terms is self-consistent. 
For this we have to prove that the nonlinear terms remain uniformly negligible for large time. We then use the asymptotic results for the linearized equation derived in section (2). We thus have, for any $k$

$$
\psi_{k, L}(y, t) \underset{t \rightarrow \infty}{\sim} \frac{\omega_{k, L, \infty}(y)}{\left(i k U^{\prime}(y)\right)^{2}} \frac{\exp (-i k U(y) t)}{t^{2}} \text { and } \omega_{k, L}(y, t) \underset{t \rightarrow \infty}{\sim} \omega_{k, L, \infty}(y) \exp (-i k U(y) t),
$$

where the subscript $L$ refers to the evolution according to the linearized dynamics. We call a quasilinear approximation to the right hand side of equation (41), the approximation where $\psi_{k}$ and $\omega_{k}$ would be evaluated according to their linearized evolution close to the base flow $U(y)$. From (42), one would expect at first sight that this quasilinear approximation of the nonlinear term $N L_{Q L}$, would give contributions of order $O(1 / t)$. The detailed computation, easily performed from (42), actually shows that the contributions of order $O(1 / t)$ identically vanish for large times. Then

$$
\epsilon N L_{k, Q L} \underset{t \rightarrow \infty}{=} O\left(\frac{\epsilon}{t^{2}}\right)
$$

This is an important remark, as it proves that within a quasilinear approximation, the contribution of the nonlinear terms $N L_{Q L}$ remains uniformly bounded, and more importantly it is integrable with respect to time.

Then it is natural to conjecture that the contribution of the nonlinear terms remains always negligible. More precisely, we naturally conjecture, that within the fully nonlinear equation, for sufficiently small $\epsilon$ :

$$
\psi_{k}(y, t) \underset{t \rightarrow \infty}{\sim} \frac{\omega_{k, \infty}(y)}{\left(i k U^{\prime}(y)\right)^{2}} \frac{\exp (-i k U(y) t)}{t^{2}} \text { and } \omega_{k}(y, t) \underset{t \rightarrow \infty}{\sim} \omega_{k, \infty}(y) \exp (-i k U(y) t)
$$

with

$$
\omega_{k, \infty}(y)=\omega_{k, L, \infty}(y)+O(\epsilon)
$$

A similar reasoning in order to evaluate the nonlinear evolution for the profile $U(y)$ would lead to the conclusion that for large times

$$
\Omega_{0}(y, t) \underset{t \rightarrow \infty}{\sim}-U_{\infty}^{\prime}(y) \text { with } U_{\infty}^{\prime}(y)=U(y)+\delta U(y),
$$

where $\delta U=O\left(\epsilon^{2}\right)$.

This means that the parallel flow will quickly stabilize again towards another parallel flow which is close to the initial one.

A natural question would be to compute the modified profile. The preceding analysis leads to the quasi-linear expression

$$
\delta U(y)=-\epsilon^{2} \int_{0}^{\infty} d t N L_{0, Q L}(t)+o\left(\epsilon^{2}\right) .
$$

This expression involves integrals over times of the linearized Euler equation. It is not amenable to a simple expression, but could be easily be computed numerically from Laplace tools.

This result has to be contrasted with the results usually obtained using a quasi-linear approach, for instance in the kinetic theory of particle dynamics (point vortex models, plasma physics, astrophysics). Usually the integrals occurring in (43) diverge. Then one invokes a time scale separation, and the divergence of the integral is regularized using a multiple scale analysis. Here, by contrast, the integral converges. This means that there is a single time scale over which all quantities reach their asymptotic value (a typical time scale here is $1 / s$ where $s$ is the typical shear). The nonlinear evolution is thus very brief and leads to very small changes in the initial profile $U$.

A theory for the relaxation towards equilibrium for the 2D Euler equations has been proposed based on a quasi-linear theory coupled to some Markovianization hypothesis, by analogy with the kinetic theory of point vortices [20]. Such an approach based on analogies are natural guess that rely on theoretical hypothesis (quasi-linear hypothesis and Markovian hypothesis), that would benefit from either theoretical justifications or numerical verifications. The results of this paper for the linearized dynamics and the discussion above, show that the approximation of the non-linear dynamics by the linearized dynamics remains uniformly self consistent, and a quasi-linear approach self-consistent. However as discussed above, the relaxation is then extremely rapid and all quantities relax rapidly. Any further assumptions like Markovianization seems then irrelevant. Simpler approach, like the one of [11, similar to the discussion of this section seems thus more relevant.

We thus conclude that a direct asymptotic expansion based on a quasi-linear approach, similar to the one in [11] or may be with more subtle treatments of the lower order contributions, is most probably the relevant 
approach for a theory of the relaxation of the 2D-Euler equations. The exact results on the linearized dynamics of the previous section and the discussion above put such an asymptotic expansion on a more rigorous basis, by proving that the leading order contribution remains self-consistently bounded for all times, and explaining why an asymptotic expansion possibly converges. In the Lundgren approach, the asymptotic behavior is described by an asymptotic expansion for large times, whose leading order term is not determined. It actually depends on the initial condition and can not be predicted only with a large time asymptotic expansion. The further interest of the Laplace method developed in this paper is to give precise predictions for the asymptotic profile that can be easily computed numerically from the Laplace tool. The numerical computations of next section will confirm the statements on this last paragraph, by showing excellent agreements between direct numerical simulation of the Euler equations on one hand and the prediction of such a simple quasi-linear approach, based on Laplace tools computations.

We also conclude that, for any profile $U$ verifying the hypothesis of this work (no unstable and no neutral modes for the linearized dynamics) with any perturbation corresponding to a small vorticity, the assumption that the velocity converges for large times towards a new parallel velocity profile close to the initial profile $U$ is a self-consistent hypothesis. We see in section 4 that this is confirmed by numerical computations.

From this discussion, we thus conclude that it is natural to conjecture that for any profile $U$ verifying the hypothesis of this work (no unstable and no neutral modes for the linearized dynamics), for any perturbation corresponding to a small vorticity, the velocity converges for large times towards a new paralleled velocity profile close to the initial profile $U$. A possible theorem expressing this more precisely would be similar to the one recently obtained by Mouhot and Villani [46, for the Landau damping in the very close setup of the Vlasov equation. It has however to be noted that no proof of such a theorem for the Euler equations is known yet, even in the simplest case of a profile $U$ without stationary points.

It is thus very natural to conjecture that the ensemble of shear flows without unstable or neutral modes is asymptotically stable 4 in the sense given previously (initial perturbation controlled by a vorticity norm, for instance the enstrophy and large time perturbation controlled in kinetic energy norm) 5 .

\section{The Kolmogorov flow}

In this section, we consider the particular case of the $2 \mathrm{D}$ Euler equations in a doubly periodic domain $\mathcal{D}=$ $[0,2 \pi / \delta)[0,2 \pi)$, where $\delta>1$ is the aspect ratio ; with the Kolmogorov base flow $U(y)=\cos (y)$.

\subsection{Stability}

In this section we study the stability of the Kolmogorov flow for 2D Euler equation dynamics. We note that, in the linearized 2D Navier-Stokes equations, Mishalkin and Sinai [45] have found that the Kolmogorov flow is stable for $\delta>1$ and unstable for $\delta<1$. Here we prove the stability for $\delta>1$ for the dynamics of both the Euler and linearized Euler equations. We also show numerically that unstable modes exist for $\delta<1$, while no stable mode exists for $\delta>1$ for the linearized 2D Euler equations.

\subsubsection{Lyapounov stability}

We consider initial vorticity conditions close to the base flow vorticity $\omega_{0}(y)=\sin (y)$. We let this initial condition evolve according to the nonlinear Euler equations (3). If the perturbation to the initial flow remains small for all times, the flow is said to be Lyapounov stable. We first prove that the base flow $\omega_{0}(y)$ is Lyapounov stable as soon as $\delta>1$.

\footnotetext{
${ }^{4}$ We refer here to the notion of asymptotic stability of an ensemble of steady states of an infinite dimensional Hamiltonian equations, see for example the work 52 where any stable soliton of the KDV equations, slightly perturbed, is proved to converge for large times towards another slightly different soliton. Asymptotic stability of ensemble of steady states has also been proved for other solutions of infinite dimensional Hamiltonian systems.

${ }^{5} \mathrm{~A}$ classical argument, presented in a rigorous framework by Caglioti and Maffei [17] in the context of the Vlasov equation, implies that steady states of the Vlasov equation for which Landau damping would occur, would be unstable in a weak norm. At the core of the argument lies the time reversal symmetry of the equations. These arguments would be easily generalized to the Euler equations. This may seem in contradiction with the notion of asymptotic stability discussed here. However the notion of stability discussed by Caglioti and Maffei involves weak topology for both the initial conditions and final state. There is no contradiction with our definition of asymptotic stability, as we control here the initial perturbation in a vorticity norm and control the convergence in a velocity norm.
} 
The classical Energy-Casimir method proposed by Arnold [2] cannot be applied directly here. Indeed, the Kolmogorov flow does not verify the hypothesis for any of the two Arnold theorems [2. However, we can still prove the stability in this case, by direct analysis. Let us define the energy-Casimir functional $F$ as

$$
F[\Omega]=\frac{1}{2} \int_{\mathcal{D}}\left(\Omega^{2}-\mathbf{V}^{2}\right)
$$

$F$ being half the enstrophy minus the kinetic energy, it is a conserved quantity for the Euler equations.

We first prove that the base flow $\omega_{0}(y)=\sin (y)\left(\mathbf{v}_{0}=\cos (y) \mathbf{e}_{x}\right)$ is a minimum of $F$. We consider the perturbation vorticity $\omega=\Omega-\omega_{0}$, and decompose it into Fourier modes $\omega=\sum_{k>1} \omega_{k} e_{k}$ with $\Delta e_{k}=-\lambda_{k} e_{k}$, where the $\lambda_{k}>0$ are arranged in increasing order, and where $e_{k}$ are orthonormal $\left(\int_{\mathcal{D}} e_{k} e_{k^{\prime}}=\delta_{k k^{\prime}}\right)$. On the doubly periodic domain $\mathcal{D}=[0,2 \pi / \delta)[0,2 \pi)$, if $\delta>1$, we have $\lambda_{1}=\lambda_{2}=1$, corresponding for instance to the modes $\cos (y)$ and $\sin (y)$. Then for any $k \geq 3, \lambda_{k}>1$.

We obtain

$$
F=\frac{1}{2} \sum_{k} \frac{\lambda_{k}-1}{\lambda_{k}} \omega_{k}^{2} .
$$

Since $\lambda_{k} \geq 1, F \geq 0$. Moreover $F\left[\omega_{0}\right]=0$. We thus conclude that $\omega_{0}$ is a global minimum for $F$.

We note that this minimum is degenerate, as all vorticity fields $\omega=\alpha \cos (y)+\beta \sin (y)$ are also minima.

Since $F$ is a conserved quantity, we conclude that

$$
\frac{1}{2} \sum_{k \geq 3} \frac{\lambda_{k}-1}{\lambda_{k}} \omega_{k}^{2}(t)=\varepsilon_{F},
$$

where $\varepsilon_{F}=F(0)$ is the small value of $F$ for the initial perturbation. Then if they are initially small, all $\omega_{k}$ for $k \geq 3$ remain small for large times, the amplitude being measured according to the norm (44).

Expression (45) does not control the first Fourier modes $\Omega_{1}$ and $\Omega_{2}$. For this, we use the fact that the enstrophy

$$
\Gamma_{2}=\int_{\mathcal{D}} \Omega^{2}
$$

is conserved. We suppose that its initial value is $\Gamma_{2,0}+\epsilon_{\Gamma}$ where $\Gamma_{2,0}$ is the base flow enstrophy and $\epsilon_{\Gamma}$ is the perturbation enstrophy. Using the enstrophy conservation we have

$$
\Omega_{1}^{2}(t)+\Omega_{2}^{2}(t)=\Gamma_{2,0}+\epsilon_{\Gamma}-\sum_{k} \omega_{k}^{2}
$$

Then, using that $\sum_{k \geq 3} \omega_{k}^{2} \leq \frac{2 \lambda_{3}}{\lambda_{3}-1} \epsilon_{F}$ (derived from (45), using $\lambda_{k} \geq \lambda_{3}$ for $k \geq 3$ ), we have

$$
\left|\Omega_{1}^{2}(t)+\Omega_{2}^{2}(t)-\Gamma_{2,0}\right| \leq \max \left\{\epsilon_{\Gamma}, \frac{2 \lambda_{3}}{\lambda_{3}-1} \epsilon_{F}\right\} .
$$

This means that the flow associated to the two first mode is $a(t) \sin (y+\phi(t))$ where $\phi$ may be arbitrary but where the amplitude $a$ is controlled up to an error of order $\max \left\{\epsilon_{\Gamma}, \epsilon_{F}\right\}$.

We have thus proved that any initial condition close to the initial profile $\omega=\sin (y)$ remains close to the family of profiles $\sin (y+\phi)$. Then the flow is Lyapounov stable in this sense.

\subsubsection{Linear and spectral stability}

Next, we let the initial conditions close to the base flow $\omega_{0}(y)=\sin y$ evolve according to the linearized 2D Euler equations (4). If the perturbation to the initial flow remains small for this dynamics, the flow is said to be linearly stable.

We decompose the perturbation vorticity in Fourier series for the $x$ variable only. For parallel flows, due to the translational invariance, such Fourier modes are independent. The modes with no dependence on $x$ are easily shown to be neutral. Then the only issue is about the stability of other modes. In order to prove this, we simply note that the perturbed Energy-Casimir functional (44) is conserved not only by the nonlinear Euler dynamics but also by the linearized one. Then, because the Energy-Casimir functional is positive, this proves that any $x$-dependent perturbation remains small if it is initially small. The flow is thus linearly stable as soon as $\delta>1$.

If the linear equation has no exponentially growing modes, it is called spectrally stable. Linear stability implies spectral stability (the converse may be wrong). Then because it is linearly stable, we can conclude that no unstable modes exist to the linearized 2D Euler equation as soon as $\delta>1$. 


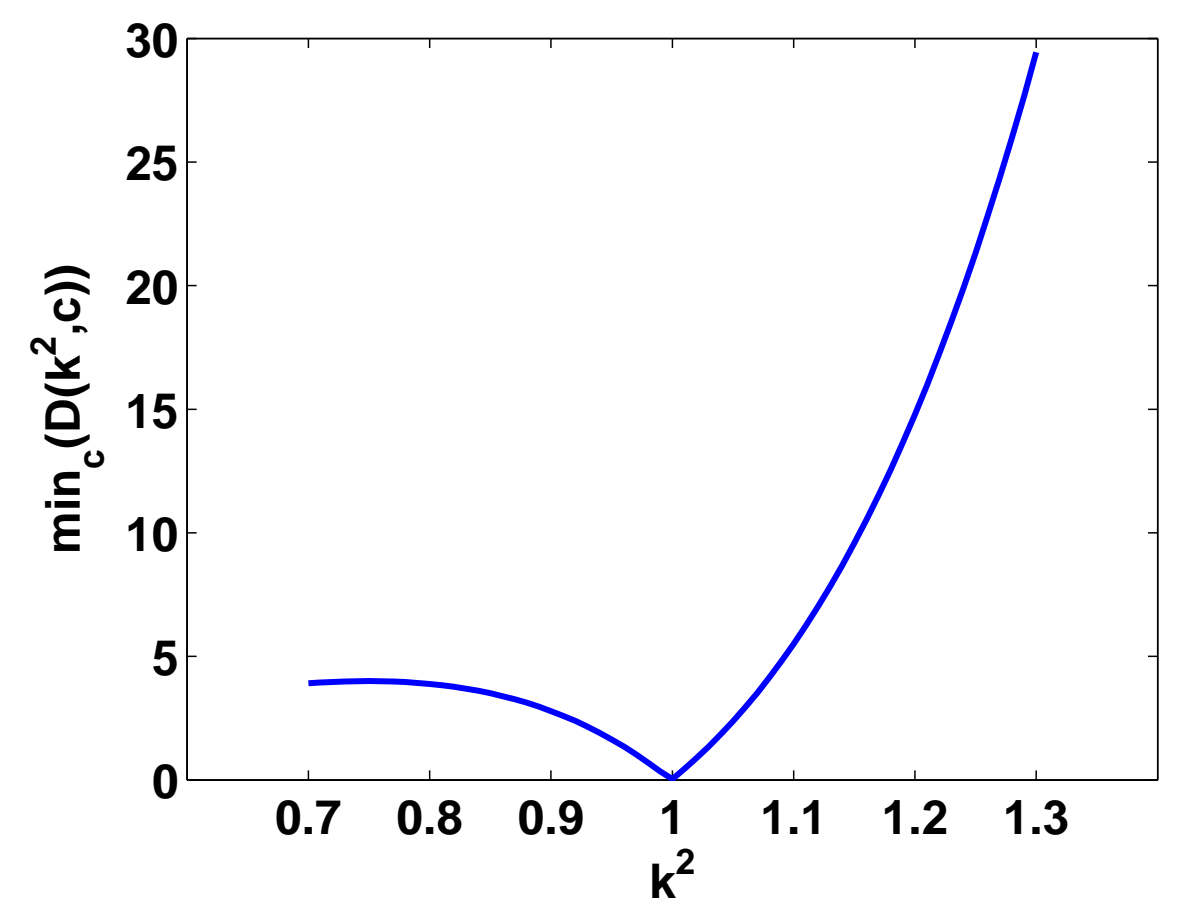

Figure 6: Minimum values for the dispersions relation $\min _{c} D^{+}(c, k)$ as a function of $k^{2}$. This plot shows that neutral modes exist only for $k^{2}=1$.

\subsubsection{Neutral modes}

We look for the modes of equation (44) such that the stream function is of the form $\psi=\phi(y) \exp (i k(x-c t))$. Then $\phi$ satisfies the classical Rayleigh equation (10).

As discussed in section 4.1.2 no unstable eigenvalue exists for $k^{2}>1$. Thus only for real values of $c$ can the Rayleigh equation have solutions for $k^{2}>1$. In the following, using numerical simulations, we show that no neutral modes exist, except for the marginal case $k=1$.

When $c$ is in the range of $U:-1=\min _{y}\{U(y)\}<c<1=\max _{y}\{U(y)\}, U-c$ vanishes at the two critical layers defined by $U\left(y_{l_{1,2}}\right)=\cos \left(y_{l_{1,2}}\right)=c$. The Rayleigh equation then has logarithmic singularities. As discussed in section 2.1, when initial value problems are considered, the relevant solutions to the Rayleigh equation are the ones that are obtained with $c^{\prime}=c+i \epsilon, c$ real, and by considering the limit $\epsilon \rightarrow 0^{+}$. We study the existence of modes in that limit.

For this, we numerically compute the dispersion relation $D_{+}(c, k)$ of the Rayleigh equation, as defined in section 2.3.2. Neutral modes correspond to zeros of $D_{+}$. We use the same numerical tools as the one described in the end of section 2.3.2. we use the Matlab function ode45, and fix the relative error parameter and the absolute error parameter of this function to $10^{-13}$, then obtain solutions for which errors in the Wronskian $W$ are typically smaller than $10^{-6}$ for $\epsilon=10^{-4}$. We approximate $D_{+}(c, k)$ by the numerically computing $D(c+i \epsilon, k)$ with $\epsilon=10^{-4}$.

Figure6 shows $D_{m}(k)=\min _{c} D^{+}(c, k)$ (we note that $D^{+}$is unchanged when the sign of $k$ is changed). For a given value of $k$, some neutral mode exist if and only if $D_{m}(k)$ vanishes. We conclude from this plot that neutral modes exist only for the value $k^{2}=1$ (we have tested values of $k^{2}$ up to $k^{2}=10$ ).

For $k^{2}=1$, we see numerically that a mode exists for $c=0$ only. The mode is then found by direct integration of equation (10). It is the trivial mode $\phi=C$ where $C$ is any constant number $(\psi=C \exp (i k x)$ and $\psi=C \exp (-i k x))$.

The representation of the complex curve $D_{+}(c)$ when $c$ is varied is called a Nyquist plot (see [48] or [5] in the context of fluid dynamics). It is very useful, as the algebraic number of loops of the Nyquist plot around 0 counts the number of unstable modes on the complex half plane $c=c_{R}+i \lambda$ with positive $\lambda$ [48, 5]. 


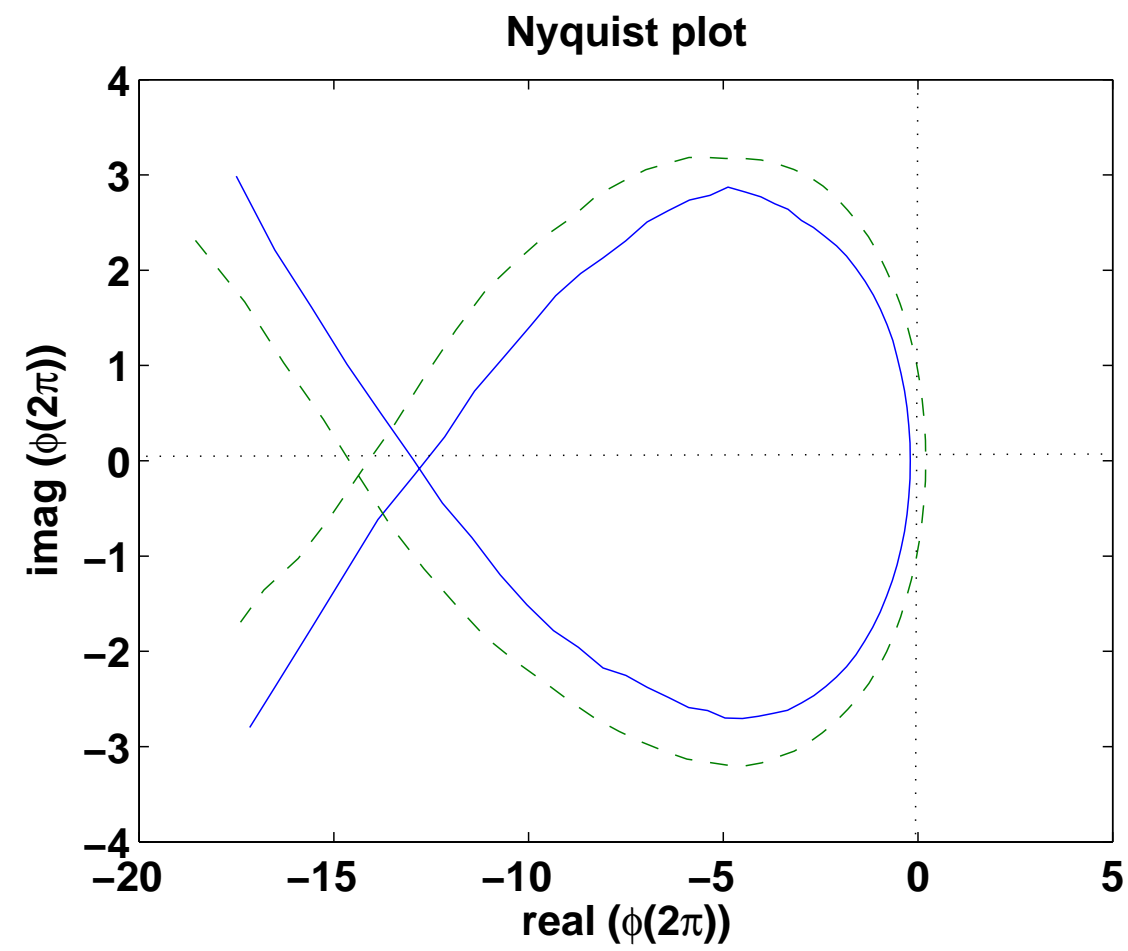

Figure 7: Nyquist plots (complex $D^{+}(c)$ represented in the complex plane, when $c$ is varied), for values $k^{2}=0.99$ (green dashed line) and $k^{2}=1.01$ (plain blue line).

Figure 7 shows the Nyquist plot of $D_{+}(c)$, for $k=0.99$ and $k=1.01$ respectively. One clearly sees the passing of the curves across the value $D_{+}=0$ when $k$ is changed from $k=0.99$ to $k=1.01$, corresponding to neutral modes for $k=1$. Moreover, we conclude that only one neutral mode exists for this value of $k$, because only one branch of the curve passes through 0 . For larger value of $c$ (not shown), the upper part of the plot loops to the right on the upper half plane goes down to the lower half plane by crossing the real axis for very large values of $\phi$, before to close on the branch visible in the lower half plane. Counting the algebraic number of loops around zero, we thus conclude that for $k>1$, no unstable modes exists, in accordance with the result of section (4.1) ; whereas for $k<1$ only one unstable mode exists.

From this analysis, we thus conclude that only one neutral mode exists. This modes corresponds to the destabilization of the flow and the appearance of an unstable mode, when passing from values $k^{2} \geq 1$ to values $k^{2} \leq 1$. It is the trivial mode $\psi=C \exp (i x)$, found for the phase speed $c=0$.

\subsection{Direct numerical computation of the 2D-Euler equations}

In this section, we illustrate and complement the above results through the direct numerical simulation of the $2 \mathrm{D}$ (nonlinear) Euler equations, in the doubly periodic domain $\mathcal{D}=[0,2 \pi / \delta)[0,2 \pi)$, for the Kolmogorov base flow $U(y)=\cos y$.

Since the base flow is homogeneous in the $x$ direction, the dynamics of the fields would be decoupled into that of the components of the form $f_{k}(y, t) \exp (i k x)$, if the equations were linearized. Then it is natural to consider fields of this form also in the (nonlinear) Euler equations, because we are interested in slightly perturbed parallel flows.

Since, in the Euler equations, instabilities are mainly large scale ones, the flow is more likely affected by large scale perturbations. Moreover, in geophysics and experiments, forcing and perturbations tend to be dominantly effective on the largest scales of the flow. It is thus natural to study initial perturbations of the form

$$
\omega(x, y, 0)=\epsilon A(y) \cos \delta x
$$

where $k=\delta$ is the smallest wavenumber (largest scale). We consider the case $\epsilon \ll 1$; we use $\epsilon=0.01$ throughout in the following numerical computations. 


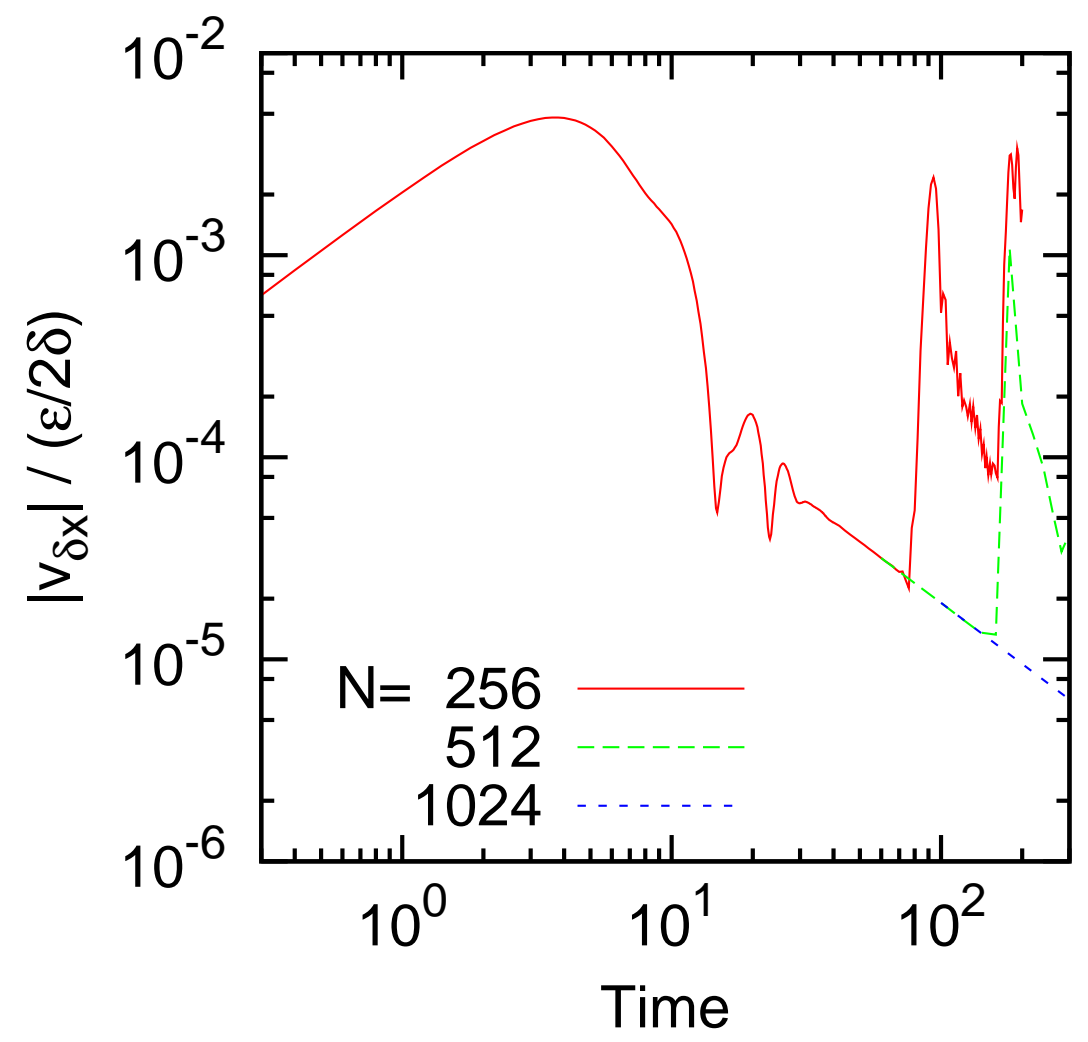

Figure 8: The time series of perturbation velocity components $\left|v_{\delta, x}(y=0, t)\right|$, for the initial perturbation profile $A(y)=1$ and the aspect ratio $\delta=1.1$, with various resolutions of the system $(N \times N)$. The simulations blow up at $t \approx 70$ for $N=256$ and at $t \approx 150$ for $N=512$. In the following numerical results, we have used high resolution enough to confirm that the asymptotic behavior is robustly observed.

Similarly, we first examine the dynamical response for the same wave number as the initial perturbation, namely,

$$
\begin{aligned}
& \omega_{\delta}(y, t)=\int \frac{\mathrm{x}}{2 \pi \delta^{-1}} \mathrm{e}^{-\beta \delta x} \omega(x, y, t) \\
& \boldsymbol{v}_{\delta}(y, t)=\int \frac{\mathrm{x}}{2 \pi \delta^{-1}} \mathrm{e}^{-\beta \delta x} \boldsymbol{v}(x, y, t) .
\end{aligned}
$$

The analysis of nonlinear effect will be performed at the end of this section.

Direct numerical simulations. In the following, for the direct numerical simulations, we use the classical pseudo-spectral method algorithm [36], which is the most precise and robust numerical algorithm currently known for the Euler and Navier Stokes equations in doubly periodic domains, and indeed regarded as the standard method for precise numerical simulations in these cases.

A standard way to compute numerical solutions to the Euler equations is to integrate directly the truncated Euler equations without viscosity [33]. We follow this procedure. The numerical scheme is thus not stable in the long run; on account of the lack of explicit dissipation, the simulation blows up when the small scale structures become of the same size as the grid scale 33. As an illustration, figure 8 presents the computation of the first mode of the velocity perturbation, computed with three different numerical simulations using $256^{2}, 512^{2}$ and $1024^{2}$ Fourier components, respectively. This illustrates the blowup after some times, occurring later on for larger resolutions. Moreover this shows that the short time numerical results are stable when the resolution is increased. In the following numerical results, we have always confirmed that the resolution of the system is high enough, by systematically testing the stability of the results by changing the resolution, such that the 
asymptotic behavior discussed is robustly observed. This procedure for assessing the numerical results for the Euler equations without dissipation is also a standard one (see for instance [33]).

An independent assessment of the quality of the numerical results is provided by figure 10, discussed later in more details. On this figure, the final vorticity profile is computed by two algorithms: the just described direct numerical simulations, and the predictions from the Laplace transform (equation (34)). The results from these two completely independent algorithms are indistinguishable on figure 10. The maximum discrepancy between the two profiles on this figure is of order 0.0001 .

Asymptotic vorticity profile for even perturbations. First, we consider an initial perturbation where $A(y)$ has the same parity as the base flow. In particular, we examine $A(y)=1$.

The space-time series of $\left|\omega_{\delta}(y, t)\right|$ is shown in Figure 1, page 5, which we have already seen. Initially, it rapidly (almost exponentially) relaxes toward the final profile, $\left|\omega_{\delta \infty}(y)\right|$; in particular, it relaxes to zero at $y=0$ and $\pi$ (stationary streamlines), whereas it remains constant at $y=\pi / 2$ and $3 \pi / 2$. The rapid relaxation of the modulus $\left|\omega_{\delta}(y, t)\right|$ is in agreement with the theoretical prediction (31) that the Lundgren ansatz is asymptotically valid.

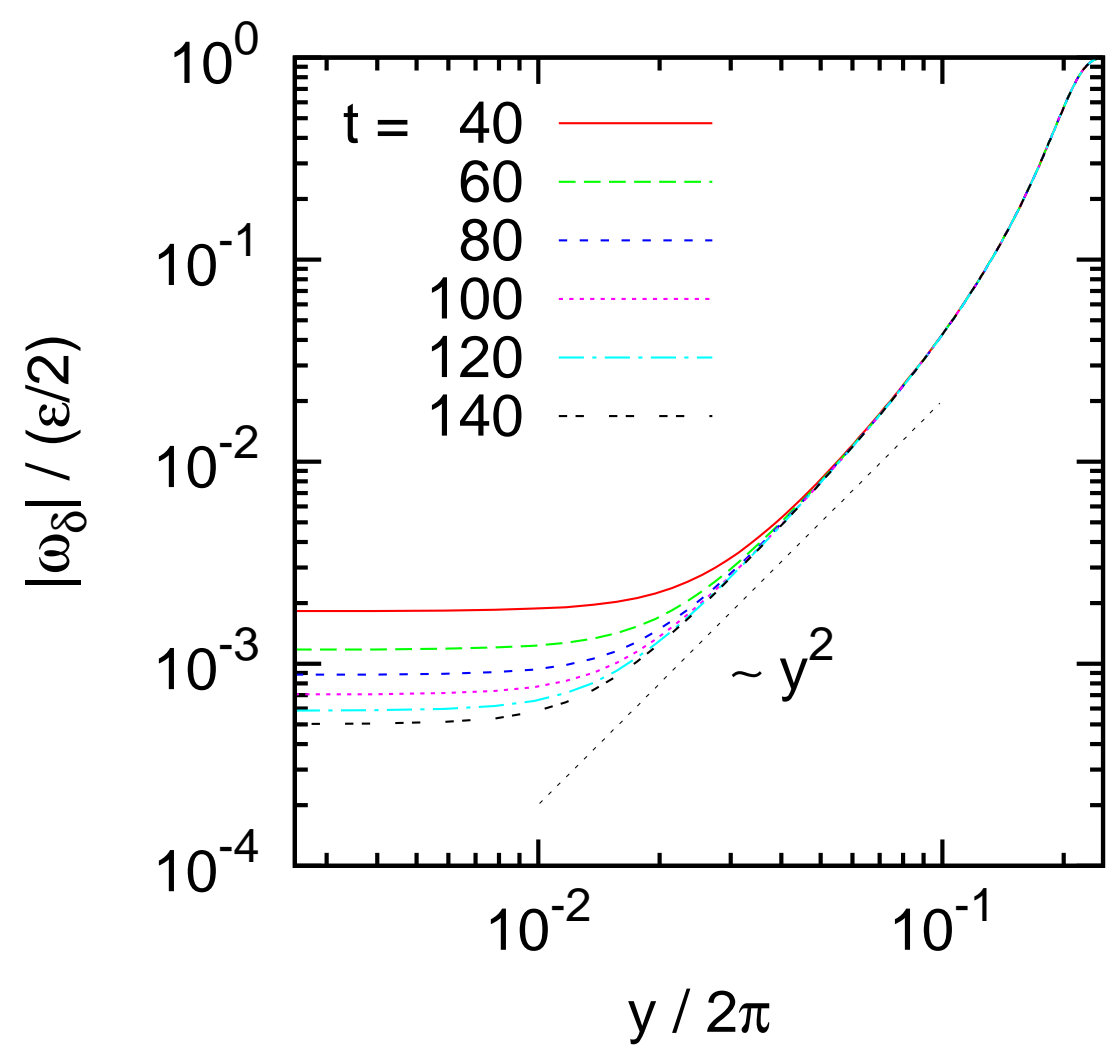

Figure 9: The profiles of perturbation vorticity, $\left|\omega_{\delta}(y, t)\right|$, at several times, for the initial perturbation profile $A(y)=1$ and aspect ratio $\delta=1.1$. A flat region is observed near the stationary streamline $y=0$. As time goes on, this flat region becomes narrower and narrower, and instead the region with the profile proportional to $y^{2}$ extends towards the stationary streamlines, leading to a parabolic profile in the large time limit.

After the rapid relaxation, $\left|\omega_{\delta \infty}(y)\right|$ converges towards the final profile algebraically. In particular, in the vicinity of $y=0$ and $\pi$ (stationary streamlines), it relaxes as slowly as $t^{-1}$, leading locally to a flat profile (see figure 91). However, as time goes on, this flat region becomes narrower and narrower, and instead the region with the profile proportional to $y^{2}$ extends towards the stationary streamlines. This indicates that, in the large time limit, the profile is parabolic in the vicinity of the stationary streamlines.

This also illustrates that the relaxation towards the stationary profile does not converge in a uniform way; the process is very slow close to the stationary streamlines whereas it is extremely rapid away from them. 


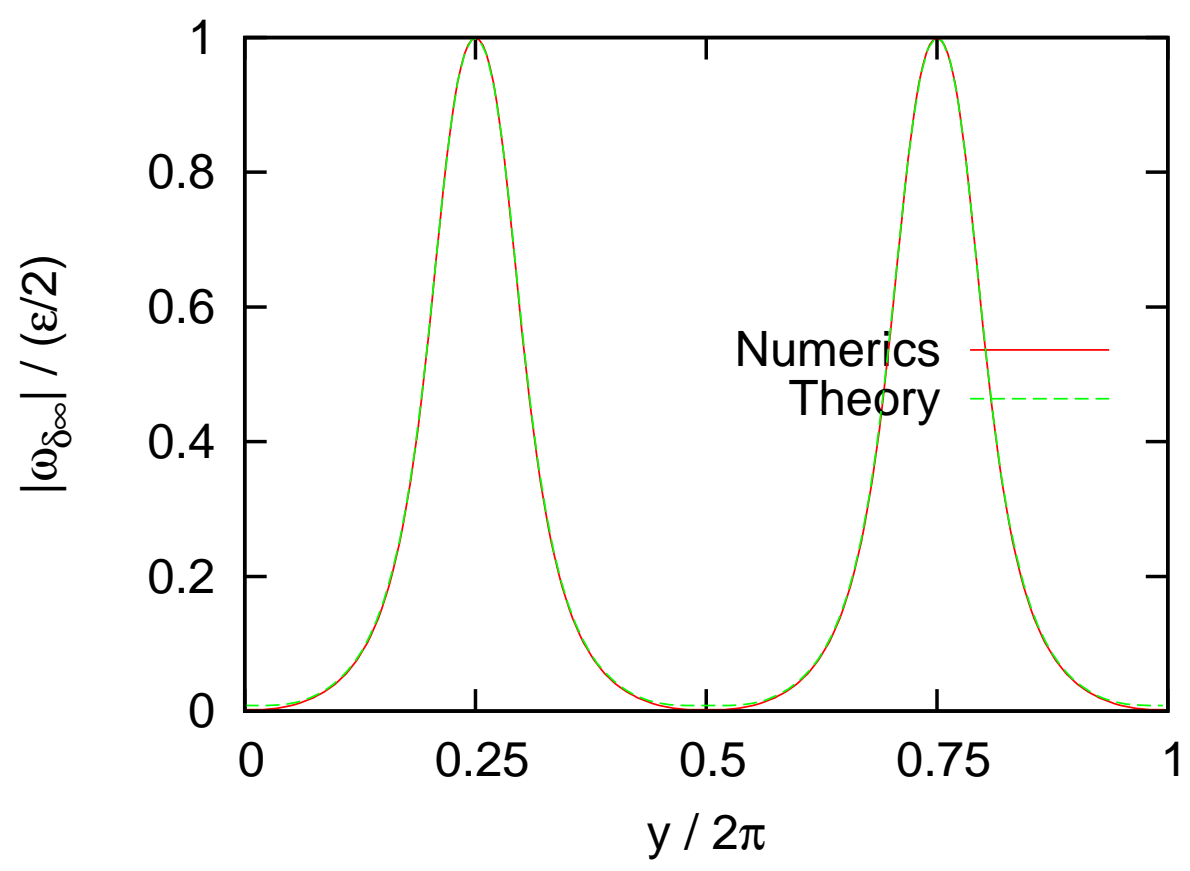

Figure 10: The final profile of the modulus of the perturbation vorticity, $\left|\omega_{\delta \infty}(y)\right|$, obtained from the direct numerical simulation and the theory (equation (34)), for the initial perturbation profile $A(y)=1$ and the aspect ratio $\delta=1.1$. The two profiles show a very good agreement.

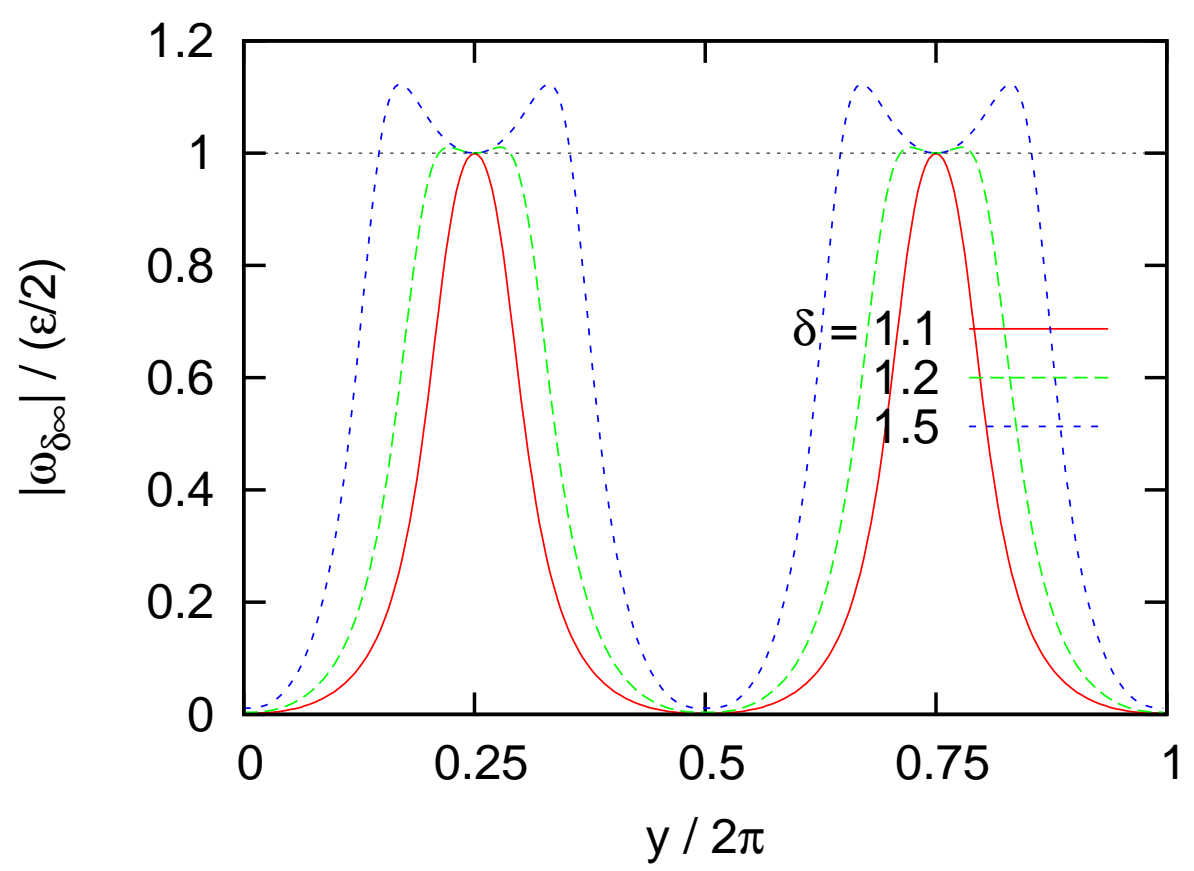

Figure 11: The final profile for the modulus of the perturbation vorticity, $\left|\omega_{\delta \infty}(y)\right|$, for the initial perturbation profile $A(y)=1$, and aspect ratios $\delta=1.1,1.2$ and 1.5, computed from the prediction of the Laplace transform tools (equation (34) ). The profile shows a bifurcation from single to double peak shapes, when $\delta$ is increased. 


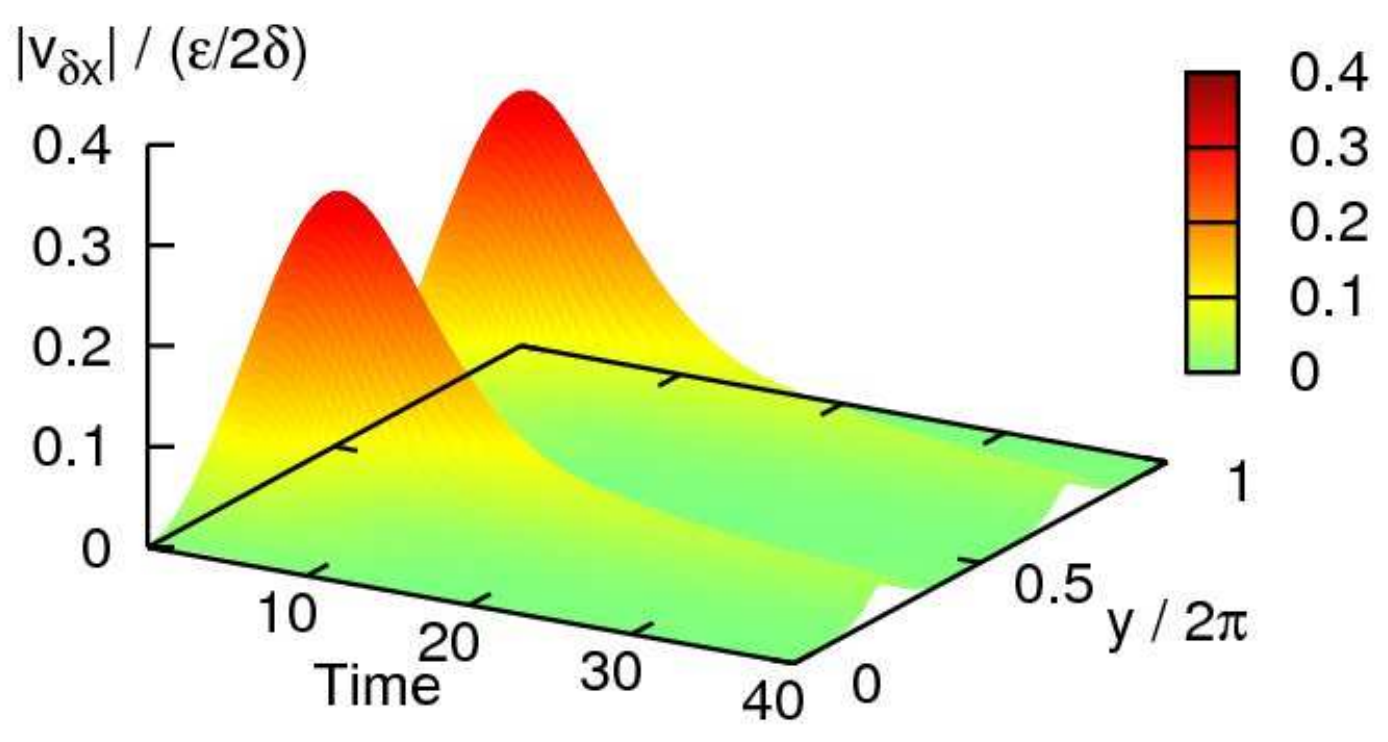

(a)

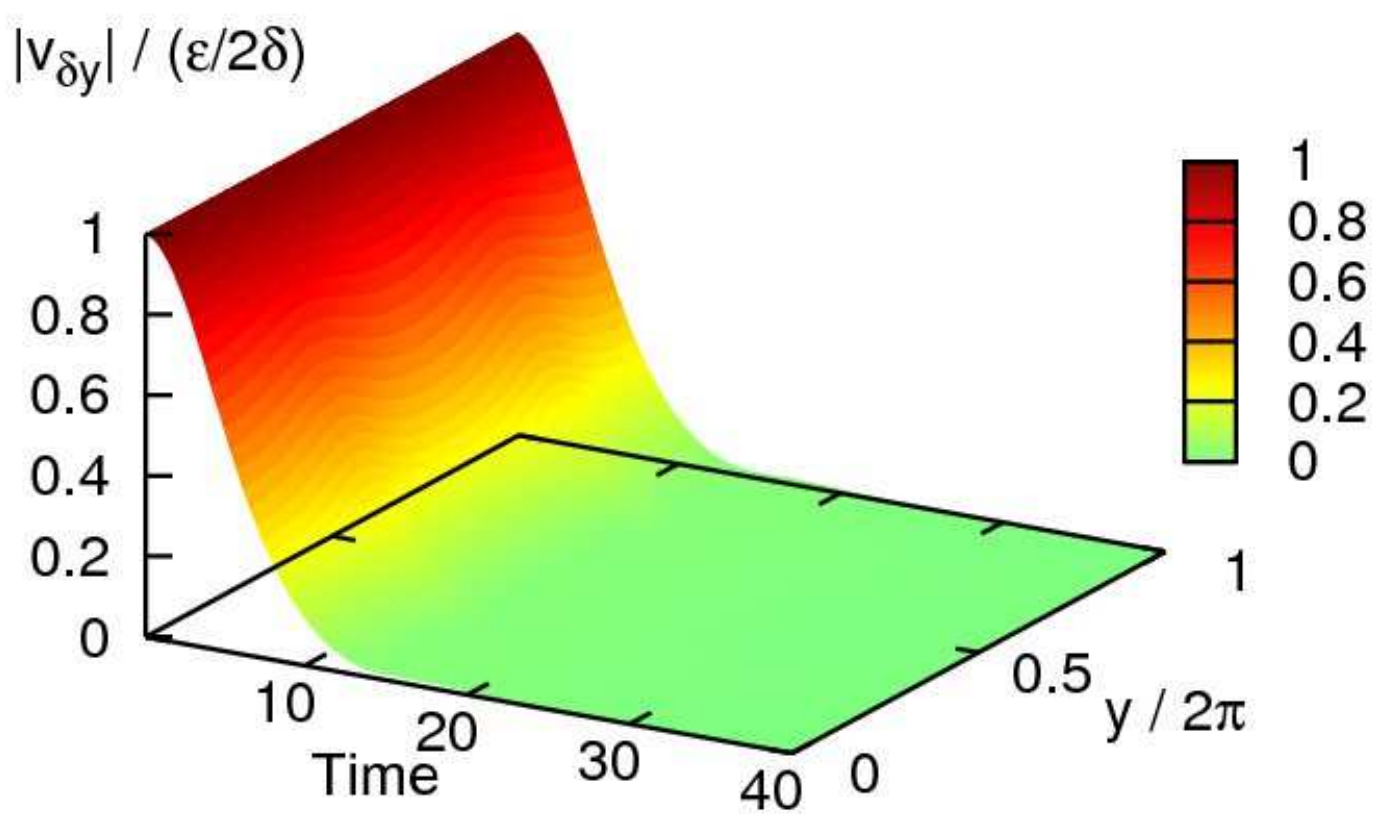

(b)

Figure 12: The space-time series of $\left|v_{\delta, x}(y, t)\right|$ (a) and $\left|v_{\delta, y}(y, t)\right|$ (b), for the initial perturbation profile $A(y)=1$ and the aspect ratio $\delta=1.1$. Both the components relax toward zero, showing the asymptotic stability of the Euler equations. 
(a)
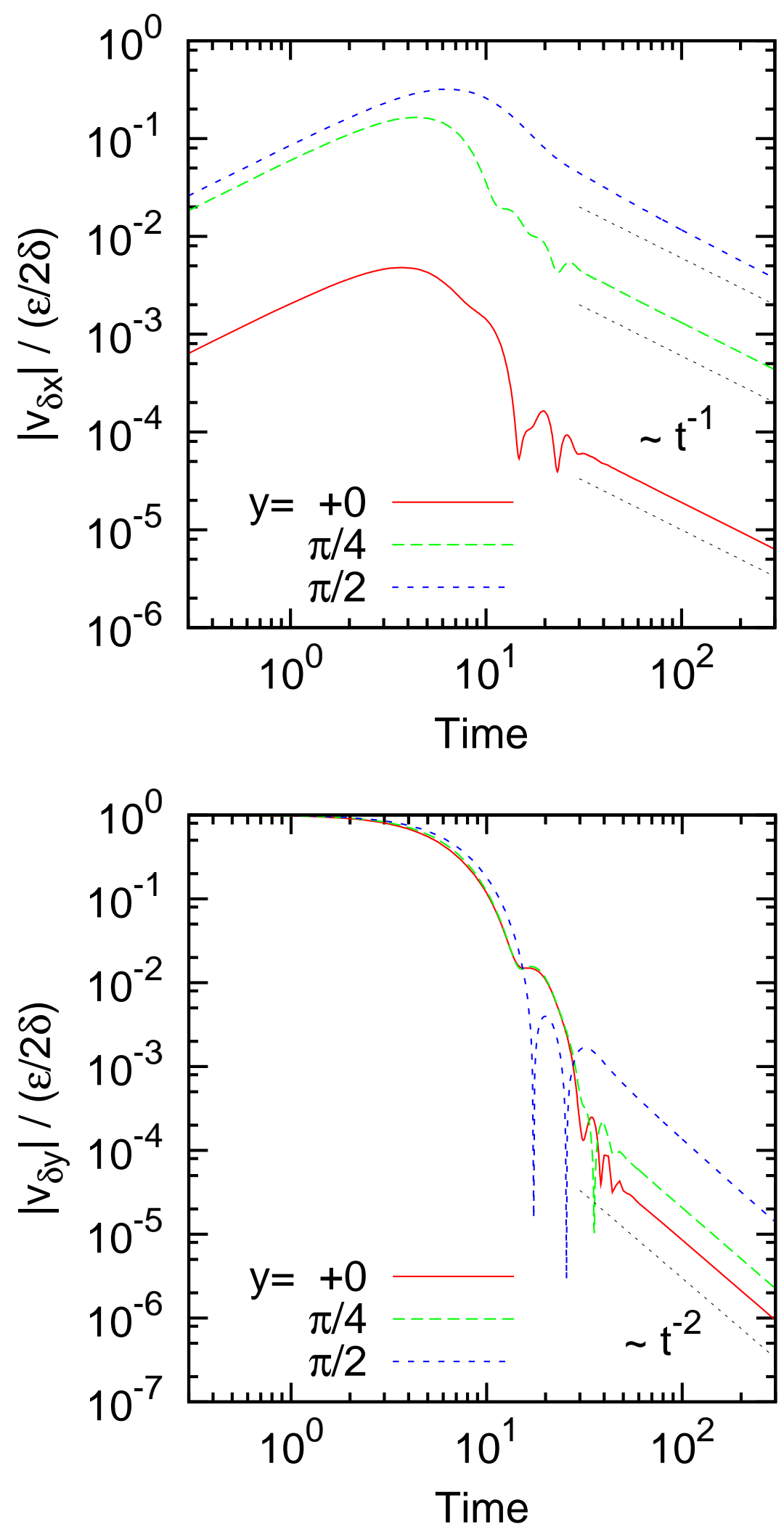

Figure 13: The time series of perturbation velocity components $\left|v_{\delta, x}(y, t)\right|$ (a) and $\left|v_{\delta, y}(y, t)\right|$ (b) at three locations, $y=0$ (vicinity of the stationary streamline) (red), $y=\pi / 4$ (green), and $y=\pi / 2$ (blue), for the initial perturbation profile $A(y)=1$ and the aspect ratio $\delta=1.1$. We observe the asymptotic forms $\left|v_{\delta, x}(y, t)\right| \sim t^{-\alpha}$, with $\alpha=1$, and $\left|v_{\delta, y}(y, t)\right| \sim t^{-\beta}$, with $\beta=2$, in accordance with the theory for the asymptotic behavior of the velocity (equations (35) and (36)) 
The width $y_{f}$ of the flat area decreases as $y_{f} \sim 1 / \sqrt{t}$, whereas the constant value of the vorticity modulus in the flat area decreases as $1 / t$. When computing the velocity from the vorticity (equation (37)), the overall effect of this flat area is thus of order $1 / t^{3 / 2}$. Such a contribution is thus of the same order as what would give the leading order term of the asymptotic expansion of oscillating integrals, as discussed in the end of section 2.3.5.

In Figure 10, we compare the final profiles obtained from the Laplace tools (equation (34)) and the direct numerical simulations. As shown, the results show a very good agreement. This agreement support both the quality of the direct numerical simulations and the results of the computation of the asymptotic profile from the Laplace transform tools.

The computation of the asymptotic profile from the Laplace method is extremely rapid and easy, compared with direct numerical simulations. Using this tool, we study some qualitative properties of the asymptotic profile. By increasing the aspect ratio $\delta$, we observe a bifurcation from a single- to a double-peaked asymptotic vorticity profile (figure 11). The three asymptotic profiles all show the depletion of the vorticity perturbation at the stationary streamlines.

Asymptotic decay of the velocity perturbation for even perturbations. The space-time series of the modulus of the perturbation velocity components $\left|v_{\delta, x}(y, t)\right|$ and $\left|v_{\delta, y}(y, t)\right|$ are shown in 12 . The relaxation to zero of the velocity perturbation illustrates the asymptotic stability of the velocity for the 2D Euler dynamics.

We investigate the asymptotic behavior of the velocity perturbation more precisely. Figure 13 shows the time series at several positions. As shown, their asymptotic forms are $\left|v_{\delta x}(y, t)\right| \sim t^{-\alpha}$, with $\alpha=1$, and $\left|v_{\delta y}(y, t)\right| \sim t^{-\beta}$, with $\beta=2$. This is in agreement with the theoretical predictions for the asymptotic behavior of the velocity perturbation (see equations 35 and 36).

Odd perturbations. Next, we consider initial perturbations where $A(y)$ has a parity opposite to the base flow one. In particular, we examine $A(y)=\sin y$.

The space-time series of $\left|\omega_{\delta}(y, t)\right|$ is shown in Figure 14, It shows an initial rapid relaxation toward the final profile, as expected from the theory (equation (34)). Since the parity of the perturbation is conserved for all time, the vorticity profile remains odd. Then $\left|\omega_{\delta}(y, t)\right|$ is zero for $y=0$ and $y=\pi$ (stationary streamlines), as expected.

The rapid relaxation is again followed by an algebraic convergence to the final profile. In particular, in the vicinity of $y=0$ and $y=\pi$ (stationary streamlines), it relaxes as slowly as, $t^{-1 / 2}$, in this case. The vorticity is always zero at the stationary streamlines, the profile in the vicinity is linear (see figure 15), not flat as in the case of even perturbations. However, as time goes on, this linear region becomes narrower and narrower, and instead the region with the profile proportional to $y^{2}$ extends towards the stationary streamlines. This indicates that, in the large time limit, the profile is locally parabolic in the vicinity of the stationary streamlines, as in the case of even perturbations. The profile being odd, we remark that such a parabolic profile means that the asymptotic vorticity profile is not twice differentiable at the stationary streamlines.

The final profile obtained from the Laplace transform tools (equation (34)) and the direct numerical simulations again show excellent agreement (see figure 16).

The space-time series of $\left|v_{\delta, x}(y, t)\right|$ and $\left|v_{\delta, y}(y, t)\right|$, similarly to the case of $A(y)=1$, shows a relaxation toward zero, illustrating the asymptotic stability of the Euler equations.

We investigate the velocity asymptotic behavior more precisely. Figure 17 shows the time series at several positions. As shown, their asymptotic forms are $\left|v_{\delta x}(y, t)\right| \sim t^{-\alpha}$, with $\alpha=1$, and $\left|v_{\delta y}(y, t)\right| \sim t^{-\beta}$, with $\beta=2$, for almost all values of $y$. Thus we verify that the direct numerical simulation for the asymptotic behavior of the velocity perturbation is in good agreement with the theory. Only in the vicinity of $y=0$ and $\pi$ (stationary streamlines), the exponents are changed to $\alpha=1.5$ and $\beta=1.5$. We recall that we have no theoretical predictions for the asymptotic velocity on these stationary streamlines.

In the last paragraphs, we have compared the mode $\omega_{\delta}$ from the direct numerical simulations of the $2 \mathrm{D}$ (nonlinear) Euler equations with the predictions of the 2D linearized Euler equations. The agreement between both is extremely good. There is indeed no visible differences, even for large times. This is in agreement with the theoretical discussion in the section 3 namely, the difference is expected to be of the order $\epsilon^{2}$. To summarize, we conclude that the asymptotic behavior of the (nonlinear) Euler equations are very well described by the quasi-linear theory discussed in the previous sections.

Evolution of the base flow profile and asymptotic stability. We now consider the evolution of the base flow profile $\Omega_{0}(y, t)$ (the $x$-average vorticity, directly related to the $x$-average velocity, please see the 
discussion preceding equation (43) page (18)). We define the difference with respect to the initial profile by $\omega_{0}(y, t)=\Omega_{0}(y, t)+U^{\prime}(y)$. We thus have

$$
\omega_{0}(y, t)=\int \frac{\mathrm{x}}{2 \pi \delta^{-1}} \omega(x, y, t) .
$$

The evolution of the base profile $\omega_{0}(y, t)$ is due to the nonlinear effects (see Eq. (43) page 18). Figure 18 shows this evolution in direct numerical simulations. This illustrates that the perturbation vorticity converges extremely rapidly (on times of order $t=15$ which correspond to the linear shear times) toward a fixed perturbation profile. The asymptotic perturbation profile is of order $\epsilon^{2}$. All these are in agreement with the theoretical discussions of section 3 .

\section{Discussion}

In this paper, we have discussed the asymptotic stability of parallel flows for the 2D Euler equations. Our results are valid for any flow that have no modes for the linearized dynamics (neither unstable nor neutral ones). This situation is a generic one, as the example of the Kolmogorov flows (section 4) illustrates. An adaptation of the present results to the case where the flow has neutral modes would be easy. Our results are valid for base flow profiles $U(y)$ with or without stationary points $y_{0}$ such that $U^{\prime}\left(y_{0}\right)=0$. We have emphasized the case with stationary points that has not been studied before.

For the linearized Euler equation, we have proven that Lundgren's ansatz (2) actually describes the asymptotic vorticity field for large times. The asymptotic vorticity field thus oscillates, for each streamline, at a multiple of the streamline frequency. The asymptotic vorticity profile depends both on the initial condition in a non trivial way, and on the base flow. The asymptotic vorticity is always strongly affected by the base flow structure, in a non-local way, especially when stationary streamlines exist. It is thus unlikely that a description based on the local shear give a good quantitative description, except may be in a limit or nearly linear shear. We have also shown that this asymptotic profile can be computed directly from the resolvent operator of the linearized Euler equation (see equation (34) and figure 11) without performing costly direct numerical computation of the Euler equations.

For the linearized Euler equations, we have also proved that the asymptotic velocity field decays algebraically for large times (equation 1), with exponents $\alpha=1$ and $\beta=2$ for any streamlines that are not stationary $\left(U^{\prime}\left(y_{0}\right) \neq 0\right)$. On the stationary streamlines, we have no theoretical predictions, but we have found numerically that two cases exist $\alpha=1$ and $\beta=2$ for perturbation vorticity fields having the same periodicity as the base flow velocity or $\alpha=3 / 2$ and $\beta=3 / 2$ for perturbation vorticity field having the opposite periodicity with respect to the base flow. Without stationary streamlines, these results are the same as the classically expected ones. With stationary streamlines, these results were unexpected as the effect of the stationary streamlines in oscillating integrals could have been expected to give $1 / \sqrt{t}$ contributions. Such contributions cancel out because of a self-consistent vorticity depletion at the stationary streamline. This is a new mechanism of vorticity depletion at the stationary streamline that we theoretically predict for the linearized 2D Euler equations and prove to be self consistent for the 2D Euler equations, and numerically confirm for the 2D Euler equations in this paper.

This vorticity depletion mechanism occurs due to the effects of the transverse component of the velocity perturbation on the background vorticity gradient. This mechanism is thus absent in cases where the background vorticity gradient identically vanishes, or for a beta-plane barotropic flow when the beta effect exactly balance the vorticity gradient, a case studied in several papers [16, 14]. We think that this last case is not generic as the vorticity depletion mechanism exists as soon as the vorticity gradient is not exactly balanced.

We use the above results to prove that if the perturbation evolves according to the linearized 2D Euler equations, the nonlinear term remains uniformly bounded in time, and actually decays algebraically for large times. Based on these results, we argue that for the Euler equations (nonlinear), a quasilinear treatment of the nonlinear terms is self consistent. This strongly suggests that such a quasilinear treatment of the nonlinear term should be valid. This also suggests that the full nonlinear equation converges towards Lundgren's type asymptotics for the perturbation vorticity field and to zero for the asymptotic velocity field, extremely rapidly.

From these theoretical arguments, we then expect that the velocity of parallel flows without unstable or neutral linear modes is asymptotically stable : the velocity converges towards a new parallel flow which is very close to the initial one, even in the absence of dissipation. The distance between the initial profile and the asymptotic one is of order $\epsilon^{2}$, where $\epsilon$ is the order of magnitude of the initial perturbation. 
Direct numerical simulations of the Euler equations close to the Kolmogorov base flow show an excellent agreement with the above theoretical predictions.

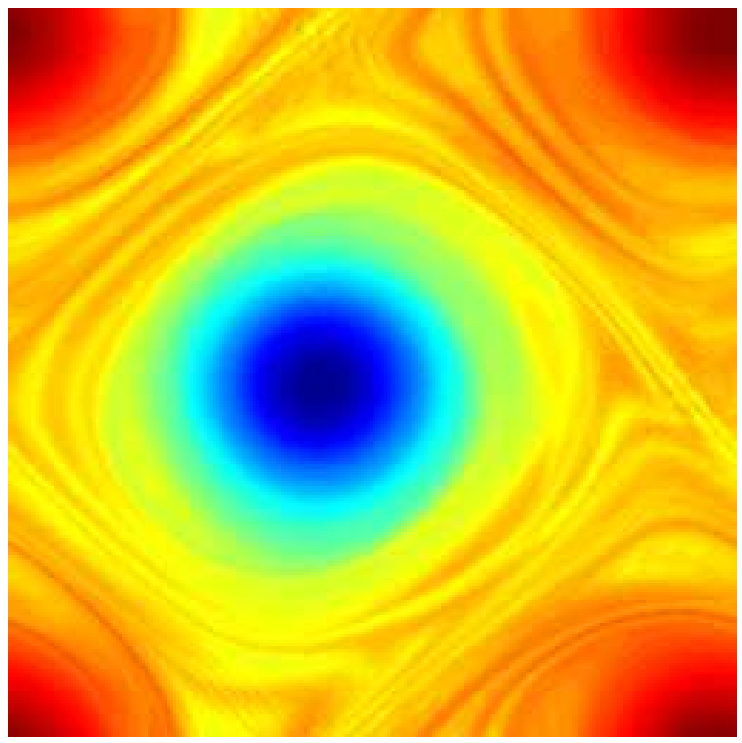

Figure 19: A snapshot of the vorticity field for the 2D Navier-Stokes equations with stochastic forces, in a statistically stationary regime. The vorticity field is close to a steady state of the Euler equation (here a dipole). The fluctuations close to this state are the visible filaments on the figure. One clearly see that such filaments are present in between the two vortices, but are absent in the core of the vortices. This is due to the vorticity depletion mechanism at the core of the vortices, the points where the angular velocity of the vortices have local extrema.

The theoretical study performed in this paper, could be easily generalized to the study of the asymptotic behavior and stability of jets in the context of both barotropic flows in the beta-plane approximations, or two dimensional axisymmetric vortices. Many recent works have considered perturbations to two dimensional vortices [67, 3, 6, 56, 58]. As far as asymptotic behavior is concerned, following this paper approach, in the case of vortices, we argue that a similar perturbation vorticity depletion should occur at any stationary point of the angular velocity of the vortex $U(r) / r$. For instance in the case of a monotonic angular velocity, such a stationary point of the angular velocity is located at the core of the vortex $r=0$, and the vorticity depletion occurs at the center of the vortex. This phenomena has indeed been observed by Bassom and Gilbert 7] (see their discussion, and the comment of their figure 2(b) and 4(a)). They have stated that "We at present lack a simple physical explanation of this process whereby vorticity is more highly suppressed than a passive scalar, and do not know whether it has applicability beyond the Gaussian vortex". The type of arguments developed in the present paper, based on the Laplace tools, suggests that such a vorticity depletion is indeed a generic phenomenon, valid for any parallel flow (resp. circular vortex), at the stationary points of the velocity profile (resp. angular velocity profile). Mathematically this is due to the regularization of the critical layer singularities at the edge of the continuous spectrum.

This vorticity depletion mechanism also impacts turbulent flows where the perturbations are locally governed by the linearized equations. Fig 19 shows a snapshot of the vorticity field in the dynamics of the stochastic 2D Navier-Stokes equations [11]. One clearly observes a depletion of the vorticity fluctuations at the core of the vortices. This effect in a stochastically forced equation is related to the depletion mechanism in a deterministic equation, described in this paper.

We have treated the linearized dynamics and the asymptotic stability for the case of parallel flows, for the 2D Euler dynamics. The generalization of such results for more complex cases, for instance flow with separatrix and stationary points would also be extremely interesting. The problem is then much more difficult from a theoretical point of view, but could be addressed numerically. Also the time dependent situation, by contrast to the case of perturbation of steady base flows, is of a large interest. It has been shown numerically that interactions with large scales dominate the small scale dynamics [25, 39, in the spirit of rapid distortion theory 
or quasilinear approaches. This has deep impact on the statistics of the associated turbulence [47.

Because both are transport equations by a non-divergent field, there is a very well known analogy between the Vlasov equations and the 2D Euler equations. An even closer relation between the 2D-Euler and Vlasov equation can also be found: the 2D -Euler dynamics of a localized perturbation (vorticity defect) is actually described by a Vlasov equation at leading order [4]. Both the 2D-Euler and Vlasov equations have very similar behaviors, including for instance relaxations without dissipation (Orr mechanism or Landau damping) and the associated asymptotic stability. The theory of the asymptotic stability of $2 \mathrm{D}$ Euler equations is thus deeply related to the asymptotic stability of Vlasov equations. We note very recent mathematical results on nonlinear Landau damping [46], a subject related to the asymptotic stability of Vlasov equations. A natural issue, is to know if such recent mathematical results [46, could be generalized to the case of the 2D Euler equations, in relation with the results obtained in the present work.

It is important to notice that a rigorous mathematical treatment of the Orr mechanism for the Euler equations, in the spirit of the work by Mouhot and Villani [46] for the Vlasov equation, does not exist yet, even in the simplest case of base flow profile $U$ without stationary point. This is a very interesting mathematical problem and we hope that some new results may follow the recent proof in the Vlasov case.

The Laplace tools we have used along this paper is suited for analytical initial data only. An interesting question is to know if similar results may hold for non analytic data. We note that for the Vlasov equations, analyticity of initial data leads to exponential decay of the perturbation ; but there are counterexamples by Glassey and Schaeffer [34, 35] showing that there is in general no exponential decay for the linearized Vlasov Poisson equation without analyticity, or without confinement. This seems to indicate that analyticity is essential for observing Landau damping for the Vlasov equation. We refer to 46 for a further discussion of this point. We guess that similarly, analyticity is an essential ingredient for the Orr mechanism in the context of the 2D Euler equations.

The current work has interesting consequences for the understanding of the kinetic theory of the point vortex model in one hand and for the linearized 2D Euler and 2D Navier Stokes equations with stochastic forces, when the large scale structures dominate the dynamics on the other hand. These applications were one of our motivation for studying the asymptotic behavior of the linearized Euler equation and asymptotic stability of the Euler equations. These consequences will be developed in forthcoming works.

\section{Acknowledgments}

We warmly thank J. Barré and E. Simonnet for useful discussions about this work.

This work was supported through the ANR program STATFLOW (ANR-06-JCJC-0037-01) and through the ANR program STATOCEAN (ANR-09-SYSC-014). 


\section{A) Oscillating integrals}

\section{A-1) General classical results}

Let us consider the asymptotic behavior, for large $t$, of the integral

$$
I(t)=\int_{a}^{b} d x g(x) \exp (i f(x) t)
$$

1. First, we consider the case when $f$ has no singular point. We suppose that $f$ and $g$ are twice differentiable, that $f$ has no singular point, i.e. $f^{\prime}(x) \neq 0$ for any $x$, and that either $g(a) \neq 0$ or $g(b) \neq 0$. Then

$$
I(t) \sim \frac{1}{i t}\left[\frac{g(b) \exp (i t f(b))}{f^{\prime}(b)}-\frac{g(a) \exp (i t f(a))}{f^{\prime}(a)}\right]
$$

Namely, $I(t) \sim t^{-1}$. This can be easily proved by integrations by part. If $g(a)=g(b)=0$, if $g$ and $f$ are sufficiently differentiable, then the asymptotic behavior can be obtained by further integrations by parts.

2. Next, we consider the case when $f$ has a single stationary point. We suppose that $f$ is a real function with a single stationary point $x_{0}\left(f^{\prime}\left(x_{0}\right)=0\right)$, that $f$ and $g$ are smooth and that $f^{\prime \prime}\left(x_{0}\right) \neq 0$. The asymptotic behavior of the oscillating integral (50) is then given by the classical stationary phase results [27, 9],

$$
I(t) \underset{t \rightarrow \infty}{\sim} g\left(x_{0}\right) \sqrt{\frac{2 \pi}{\left|f^{\prime \prime}\left(x_{0}\right)\right|}} \exp \left(\frac{i \epsilon \pi}{4}\right) \frac{\exp \left(i t f\left(x_{0}\right)\right)}{\sqrt{t}},
$$

where $\epsilon$ is the sign of $f^{\prime \prime}\left(x_{0}\right)$. Namely, $I(t) \sim t^{-1 / 2}$.

\section{A-2) Oscillating integrals and the velocity asymptotic expansion}

We apply the general methods of oscillating integrals to the particular case of the computation of the velocity from an oscillating vorticity field, like for instance the case given by equation (38). We first evaluate the long time asymptotics of

$$
\mathbf{v}(y, t)=\int d y^{\prime} \mathbf{G}_{k}\left(y, y^{\prime}\right) h\left(y^{\prime}\right) \exp \left(-i k U\left(y^{\prime}\right) t\right)
$$

where the function $h$ is assumed to be twice differentiable and where $\mathbf{G}_{k}$ is the Green function for the computation of the velocity $\mathbf{v}(y) \exp (i k x)$ from a vorticity field $\omega(y) \exp (i k x)$. We treat explicitly the case of a channel $-L<y<L$. The results are also valid for periodic boundary conditions for $y$, just by dropping all the contributions from the boundary in the expressions bellow.

Before going into more general discussions, we note that for the case of a linear base flow $U(y)=s y$, and when $h(y)$ is either constant, sinusoidal, or hyperbolic sinusoidal, then an explicit expression for $\mathbf{v}_{k}(y, t)$ is obtained (see [19]).

Clearly, equation (53) is an oscillating integral. In order to study its asymptotic expansion, we use the results of section A-1 of this appendix.

First, we treat the case of a base flow without stationary point. We use the fact that $\mathbf{G}_{k}(y,$.$) is smooth$ everywhere, except for $y^{\prime}=y$ (see appendix B). Then we can use the results on the asymptotic behaviors of oscillating integrals (section A-1) for both intervals $-L<y^{\prime}<y$ and $y<y^{\prime}<L$ independently. Moreover, we assume that $h(y)$ is at least twice differentiable. We then obtain

$v_{x}(y, t) \underset{t \gg l / k s}{\sim}-\frac{1}{i k s t}\left[G_{k, x}(y, L) h(L) \exp (-i k U(L) t)-G_{k, x}(y,-L) h(-L) \exp (i k U(-L) t)+h(y) \exp (-i k U(y) t)\right]$

the first two terms are contributions from the boundaries and the third term is due to the discontinuity of $G_{k, x}\left(y, y^{\prime}\right)$ for $y=y^{\prime}$ (see (59) in appendix B). Here we define $s$ as the minimum shear rate $s=\min \left(U^{\prime}(y)\right)$, and $l$ is a typical length scale that characterizes the variations of $h(y)$. Similarly we obtain

$v_{y}(y, t) \underset{t \gg l / k s}{\sim} \frac{1}{k^{2} s^{2} t^{2}}\left[\frac{\partial G_{k, y}}{\partial y^{\prime}}(y, L) h(L) \exp (-i k U(L) t)-\frac{\partial G_{k, y}}{\partial y^{\prime}}(y,-L) h(-L) \exp (i k U(L) t)-i k h(y) \exp (-i k U(y) t)\right]$ 
We note that there is no contribution of order $1 / t$ in this case, because $G_{k, y}\left(y, y^{\prime}\right)$ has a discontinuity of its first derivative only, for $y^{\prime}=y$ (see (60) in appendix B ; moreover in the case of a bounded domain $G_{k}$ vanishes at the boundaries $\left.\left(G_{k, y}(y, L)=0\right)\right)$.

Next, we treat the case of a base flow with stationary points $y_{m}$ such that $U^{\prime}\left(y_{m}\right)=0$. We assume that each stationary point $y_{m}$ is not degenerated $\left(f^{\prime \prime}\left(y_{m}\right) \neq 0\right)$. We perform the computation for only one of these, denoted $y_{0}$, without loss of generality.

Recall that the classical results on oscillating integrals (52) assume the function $g$ (see Eq. 50) to be smooth. This is not always the case for us. Indeed, the Green function $G_{k}\left(y, y^{\prime}\right)$ is not smooth for $y=y^{\prime}$. However, if $y \neq y_{0}$, this discontinuity for $G_{k}$ or for its derivative can easily be handled by dividing the integration interval into two subintervals, as has been done in the previous paragraph. Then we conclude that the leading order of the asymptotic behavior is still dominated by the contribution of the stationary points. Thus the result (52) is still valid.

Then, from (53), using (52), we obtain, for $y \neq y_{0}$,

$$
\mathbf{v}(y, t) \underset{t \rightarrow \infty}{\sim} \mathbf{G}_{k}\left(y, y_{0}\right) h\left(y_{0}\right) \sqrt{\frac{2 \pi}{\left|k U^{\prime \prime}\left(y_{0}\right)\right|}} \exp \left(\frac{i \epsilon_{k} \pi}{4}\right) \frac{\exp \left(-i k U\left(y_{0}\right) t\right)}{\sqrt{t}},
$$

where $\epsilon_{k}$ is the sign of $-k U^{\prime \prime}\left(y_{0}\right)$. We note that the asymptotic expansion has a discontinuity for $y=y_{0}$, due to the discontinuity of the Green function. Through a straightforward generalization of the classical results (52) to oscillating integrals with discontinuous functions $g$, we can conclude that this discontinuity is regularized over a length scale $l=\sqrt{1 /\left(\left|k U^{\prime \prime}\left(y_{0}\right)\right| t\right)}$, that decreases with time.

\section{B) Green functions}

Let us establish the expression and some properties for the velocity Green function $\mathbf{G}_{k}$. The results on the discontinuity of the Green function, equations (59) and (60) bellow, are necessary for the discussion of appendix A. We here consider the case of periodic boundary conditions ( $y 2 \pi$-periodic), though the case of a channel geometry $-L \leq y \leq L$ can be treated similarly, the resultant equations (59) and (60) remaining unchanged.

Let us denote $H_{k}$ the Green function for the stream function in the $x$-Fourier space. The $x$-Fourier transforms of $\omega=\Delta \psi$ gives $\omega_{k}=d^{2} \psi_{k} / d y^{2}-k^{2} \psi_{k}$. $H_{k}\left(y, y^{\prime}\right)$ is thus solution of

$$
\frac{\partial^{2} H_{k}}{\partial y^{2}}-k^{2} H_{k}=\delta\left(y-y^{\prime}\right)
$$

where $H_{k}\left(., y^{\prime}\right)=0$ is a $2 \pi$-periodic function for any $y$.

We note that $H_{k}\left(y, y^{\prime}\right)$, considered as function of $y$, is smooth everywhere except for $y=y^{\prime}$. For $y=y^{\prime}, H_{k}$ is continuous and has a jump unity for its first derivative:

$$
\frac{\partial H_{k}}{\partial y}\left(y^{\prime+}, y^{\prime}\right)-\frac{\partial H_{k}}{\partial y}\left(y^{\prime-}, y^{\prime}\right)=1
$$

where $F\left(y^{\prime+}, y^{\prime}\right)$ is the limit of $F\left(y, y^{\prime}\right)$ for $y^{\prime}$ going to $y$ with the condition $y>y^{\prime}$.

Because of the translational invariance in a doubly periodic domain, the set of equations (57/58) and the associate boundary conditions are invariant under translation. Then clearly, $H_{k}\left(y, y^{\prime}\right)$ depends only on $\left\|y-y^{\prime}\right\|$ where

$$
\|y\|=\min _{\text {integer } n}|2 \pi n-y|
$$

Besides these general properties, an explicit expression to $H_{k}$ can be found from (57) and (58):

$$
H_{k}\left(y, y^{\prime}\right)=-\frac{\cosh (k\|y-\pi\|)}{2 \sinh (k \pi)}
$$

Using the fact that $H_{k}\left(y, y^{\prime}\right)$ depends only on $\left\|y-y^{\prime}\right\|$ and (58), it is easily verified that $H_{k}\left(y, y^{\prime}\right)$, considered as a function of $y^{\prime}$, is differentiable and has a discontinuity in its derivative for $y^{\prime}=y$ :

$$
\frac{\partial H_{k}}{\partial y}\left(y, y^{+}\right)-\frac{\partial H_{k}}{\partial y}\left(y, y^{-}\right)=1
$$


Using $\mathbf{v}=\nabla \wedge\left(\psi \mathbf{e}_{z}\right)$, we have for the $x$-Fourier transforms : $\mathbf{v}_{k, x}=-d \psi_{k} / d y$ and $\mathbf{v}_{k, y}=i k \psi_{k}$. Thus

$$
\mathbf{G}_{k}=\left(-\frac{\partial H_{k}}{\partial y}, i k H_{k}\right)
$$

Then, using the properties of $H_{k}$, we note that $\mathbf{G}_{k}\left(., y^{\prime}\right)$ is smooth everywhere except for $y=y^{\prime}$, and that its derivative has a jump for $y=y^{\prime}$ :

$$
\mathbf{G}_{k}\left(y, y^{+}\right)-\mathbf{G}_{k}\left(y, y^{-}\right)=(-1,0)
$$

and

$$
\frac{\partial \mathbf{G}_{k, y}}{\partial y}\left(y, y^{+}\right)-\frac{\partial \mathbf{G}_{k, y}}{\partial y}\left(y, y^{-}\right)=i k
$$




\section{References}

[1] A. Antkowiak and P. Brancher. Transient energy growth for the Lamb-Oseen vortex. Physics of Fluids, 16:L1-L4, January 2004.

[2] V. I. Arnold. On an a-priori estimate in the theory of hydrodynamic stability. Izv. Vyssh. Uchebbn. Zaved. Matematika; Engl. transl.: Am. Math. Soc. Trans., 79(2):267-269, 1966.

[3] K. Bajer, A. P. Bassom, and A. D. Gilbert. Accelerated diffusion in the centre of a vortex. Journal of Fluid Mechanics, 437:395-411, June 2001.

[4] N. J. Balmforth, D. Del-Castillo-Negrete, and W. R. Young. Dynamics of vorticity defects in shear. Journal of Fluid Mechanics, 333:197-230, 1997.

[5] N. J. Balmforth and P. J. Morrison. A necessary and sufficient instability condition for inviscid shear flow. Studies in Appl. Math., 102:309-344, 1999.

[6] N. J. Balmforth, S. G. L. Smith, and W. R. Young. Disturbing vortices. Journal of Fluid Mechanics, 426:95-133, January 2001.

[7] A. P. Bassom and A. D. Gilbert. The spiral wind-up of vorticity in an inviscid planar vortex. Journal of Fluid Mechanics, 371:109-140, September 1998.

[8] L. Belenkaya, S. Friedlander, and V. Yudovich. The unstable spectrum of oscillating shear flows. SIAM Journal on Applied Mathematics, 59(5):1701-1715, 1999.

[9] N. Bleistein and R. Handelsman. Asymptotic Expansions of Integrals. Dover, New York, 1975.

[10] F. Bouchet. Simpler variational problems for statistical equilibria of the 2D Euler equation and other systems with long range interactions. Physica D Nonlinear Phenomena, 237:1976-1981, August 2008.

[11] F. Bouchet and E. Simonnet. Random changes of flow topology in two dimensional and geophysical turbulence. ArXiv e-prints, 2008.

[12] R. J. Briggs, J. D. Daugherty, and R. H. Levy. Role of Landau Damping in Crossed-Field Electron Beams and Inviscid Shear Flow. Physics of Fluids, 13:421-432, February 1970.

[13] S. N. Brown and K. Stewartson. On the algebraic decay of disturbances in a stratified linear shear flow. Journal of Fluid Mechanics, 100:811-816, 1980.

[14] G. Brunet and P. H. Haynes. The Nonlinear Evolution of Disturbances to a Parabolic Jet. Journal of Atmospheric Sciences, 52:464-477, February 1995.

[15] G. Brunet and M. T. Montgomery. Vortex Rossby waves on smooth circular vortices - Part I. Theory. Dynamics of Atmospheres and Oceans, 35:153-177, May 2002.

[16] G. Brunet and T. Warn. Rossby Wave Critical Layers on a Jet. Journal of Atmospheric Sciences, 47:11731178, May 1990.

[17] E. Caglioti and C. Maffei. Time asymptotics for solutions of vlasov poisson equation in a circle. J. Stat. Phys., 92(1):301-323, 1998.

[18] E. Caglioti, M. Pulvirenti, and F. Rousset. The 2D constrained Navier Stokes equation and intermediate asymptotics. Journal of Physics A Mathematical General, 41:H4001+, August 2008.

[19] K. M. Case. Stability of Inviscid Plane Couette Flow. Physics of Fluids, 3:143-148, March 1960.

[20] P.-H. Chavanis. Quasilinear Theory of the 2D Euler Equation. Physical Review Letters, 84:5512-5515, June 2000.

[21] P.H. Chavanis. Statistical mechanis of two-dimensional vortices and stellar systems. In T. Dauxois, S. Ruffo, E. Arimondo, and M. Wilkens, editors, Dynamics and Thermodynamics of Systems With Long Range Interactions, 2002. 
[22] L. A. Dikii. The Stability of Plane-Parallel Flows of an Ideal Fluid. Soviet Physics Doklady, 5:1179, 1960.

[23] P. G. Drazin and W. H. Reid. Hydrodynamic stability. Cambridge university press, 2004, second edition.

[24] D. H. E. Dubin and T. M. O'Neil. Two-dimensional guiding-center transport of a pure electron plasma. Phys. Rev. Lett., 60(13):1286-1289, Mar 1988.

[25] B. Dubrulle and S. Nazarenko. Interaction of turbulence and large-scale vortices in incompressible 2D fluids. Physica D, 110:123-138, 1997.

[26] R. S. Ellis, K. Haven, and B. Turkington. Nonequivalent statistical equilibrium ensembles and refined stability theorems for most probable flows . Nonlinearity, 15:239-255, March 2002.

[27] A. Erdélyi. Asymptotic expansions. Dover, New York, 1956.

[28] G. Eyink, U. Frisch, R. Moreau, and A. Sobolevski. Euler: 250 years on, volume 237 of Physica D. 2008.

[29] B. Farrell. Developing Disturbances in Shear. Journal of Atmospheric Sciences, 44:2191-2199, August 1987.

[30] B. F. Farrell and P. J. Ioannou. Generalized Stability Theory. Part I: Autonomous Operators. Journal of Atmospheric Sciences, 53:2025-2040, July 1996.

[31] B. F. Farrell and P. J. Ioannou. Structural Stability of Turbulent Jets. Journal of Atmospheric Sciences, 60:2101-2118, September 2003.

[32] S. Friedlander and L. Howard. Instability in Parallel Flows Revisited. Studies in Applied Mathematics, 101(1):1-21, 1998.

[33] U. Frisch, T. Matsumoto, and J. Bec. Singularities of Euler flow? Not out of the blue! Journal of Statistical Physics, 113:761-781, 2002.

[34] R. Glassey and J. Schaeffer. Time decay for solutions to the linearized Vlasov equation. Transport Theory and Statistical Physics, 23:411-453, February 1994.

[35] R. Glassey and J. Schaeffer. On time decay rates in Landau damping. J. Comm. Partial Differential Equations, 20:647-676, 1995.

[36] David Gottlieb and Steven A. Orszag. Numerical analysis of spectral methods: theory and applications. Society for Industrial Mathematic, 1987.

[37] E. Grenier, C. K. R. T. Jones, F. Rousset, and B. Sandstede. Viscous perturbations of marginally stable Euler flow and finite-time Melnikov theory. Nonlinearity, 18:465-483, March 2005.

[38] M. B. Isichenko. Nonlinear Landau Damping in Collisionless Plasma and Inviscid Fluid. Physical Review Letters, 78:2369-2372, March 1997.

[39] J.-P. Laval, B. Dubrulle, and S. V. Nazarenko. Fast numerical simulations of 2D turbulence using a dynamic model for subfilter motions. J. Comp. Phys., 196:184-207, 2004.

[40] S. Le Dizès. Non-axisymmetric vortices in two-dimensional flows. Journal of Fluid Mechanics, 406:175-198, March 2000.

[41] T. S. Lundgren. Strained spiral vortex model for turbulent fine structure. Physics of Fluids, 25:2193-2203, December 1982.

[42] S. R. Maassen, H. J. H. Clercx, and G. J. F. van Heijst. Self-organization of decaying quasi-two-dimensional turbulence in stratified fluid in rectangular containers. J. Fluid Mech., 495:19-33, 2003.

[43] D. Marteau, O. Cardoso, and P. Tabeling. Equilibrium states of two-dimensional turbulence: An experimental study. Phys. Rev. E, 51:5124-5127, 1995.

[44] Jonathan Miller. Statistical mechanics of euler equations in two dimensions. Phys. Rev. Lett., 65(17):21372140 , Oct 1990 . 
[45] Mishalkin and Sinai. Investigation of the stability of a stationary solution of a system of equations for the plane movement of an incompressible viscous liquid. J. Appl. Math. Mech., 25:1700-1705, 1961.

[46] C. Mouhot and C. Villani. On the Landau damping. arXiv 0904.2760.

[47] S. Nazarenko and J.-P. Laval. Non-local two-dimensional turbulence and Batchelor's regime for passive scalars. J. Fluid Mech., 408:301-321, 2000.

[48] D. Nicholson. Introduction to plasma theory. Wiley, New-York, 1983.

[49] D. S. Nolan and M. T. Montgomery. The Algebraic Growth of Wavenumber One Disturbances in Hurricanelike Vortices. Journal of Atmospheric Sciences, 57:3514-3538, November 2000.

[50] W. M. F. Orr. The stability or instability of the steady motions of a perfect liquid and of a viscous liquid. Proc. Roy. Irish Acad, pages 9-69, 1907.

[51] J. Paret and P. Tabeling. Intermittency in the two-dimensional inverse cascade of energy: Experimental observations. Phys. Fluids, 10:3126-3136, 1998.

[52] R. L. Pego and M. I. Weinstein. Asymptotic stability of solitary waves. Communications in Mathematical Physics, 164:305-349, August 1994.

[53] L. Rayleigh. On the instability of jets. Proc. Lond. Math. Soc, 10:4-13, 1879.

[54] R. Robert. A maximum-entropy principle for two-dimensional perfect fluid dynamics. J. Stat. Phys., 65:531-553, 1991.

[55] S. I. Rosencrans and D. H. Sattinger. On the spectrum of an operator occuring in the theory of hydrodynamic stability. J. Math. Phys., 45:289-300, 1966.

[56] D. A. Schecter, D. H. E. Dubin, A. C. Cass, C. F. Driscoll, I. M. Lansky, and T. M. O'Neil. Inviscid damping of asymmetries on a two-dimensional vortex. Physics of Fluids, 12:2397-2412, October 2000.

[57] D. A. Schecter, D. H. E. Dubin, K. S. Fine, and C. F. Driscoll. Vortex crystals from 2D Euler flow: Experiment and simulation. Phys. Fluids, 11:905-914, 1999.

[58] D. A. Schecter and M. T. Montgomery. On the symmetrization rate of an intense geophysical vortex. Dynamics of Atmospheres and Oceans, 37:55-88, June 2003.

[59] K. Schneider and M. Farge. Final states of decaying 2D turbulence in bounded domains: influence of the geometry. Physica D, 2008.

[60] Roman Shvydkoy and Susan Friedlander. On recent developments in the spectral problem for the linearized euler equation. In Gui-Qiang Chen, George Gasper, and Joseph Jerome, editors, Nonlinear partial differential equations and related analysis, volume 371 of Contemporary Mathematics, pages 271-295. American Mathematical Society, 2005.

[61] E. Simonnet. On the unstable discrete spectrum of the linearized 2-D Euler equations in bounded domains. Physica D Nonlinear Phenomena, 237(20):2539-2552, October 2008.

[62] R. A. Smith and M. N. Rosenbluth. Algebraic instability of hollow electron columns and cylindrical vortices. Physical Review Letters, 64:649-652, February 1990.

[63] A. Sommerfeld. Ein beitrag zur hydrodynamischen erklaerung der turbulenten fluessigkeitsbewegungen. Proceedings 4th International Congress of Mathematicians, Rome, 3:116-124, 1908.

[64] J. Sommeria. Experimental study of the two dimensional inverse energy cascade in a square box. J. Fluid. Mech., 170:139-168, 1986.

[65] W. Thomson. Rectilinear motion of viscous fluid between two parallel plates. Philos. Mag., 24:188-196, 1887.

[66] K. K. Tung. Initial-value problems for Rossby waves in a shear flow with critical level. Journal of Fluid Mechanics, 133:443-469, August 1983. 
[67] M. R. Turner and A. D. Gilbert. Linear and nonlinear decay of cat's eyes in two-dimensional vortices, and the link to Landau poles. J. Fluid Mech., 593:255-279, 2007.

[68] F. Volponi. Local algebraic instability of shear-flows in the Rayleigh equation. Journal of Physics $A$ Mathematical General, 38:4293-4307, May 2005.

[69] G. Wolansky and M. Ghil. Nonlinear Stability for Saddle Solutions of Ideal Flows and Symmetry Breaking. Commun. Math. Phys., 193:713-736, 1998.

[70] T. Yamagata. On trajectories of Rossby wave-packets released in a lateral shear flow. Journal of Oceanography, 32:162-168, 1976. 


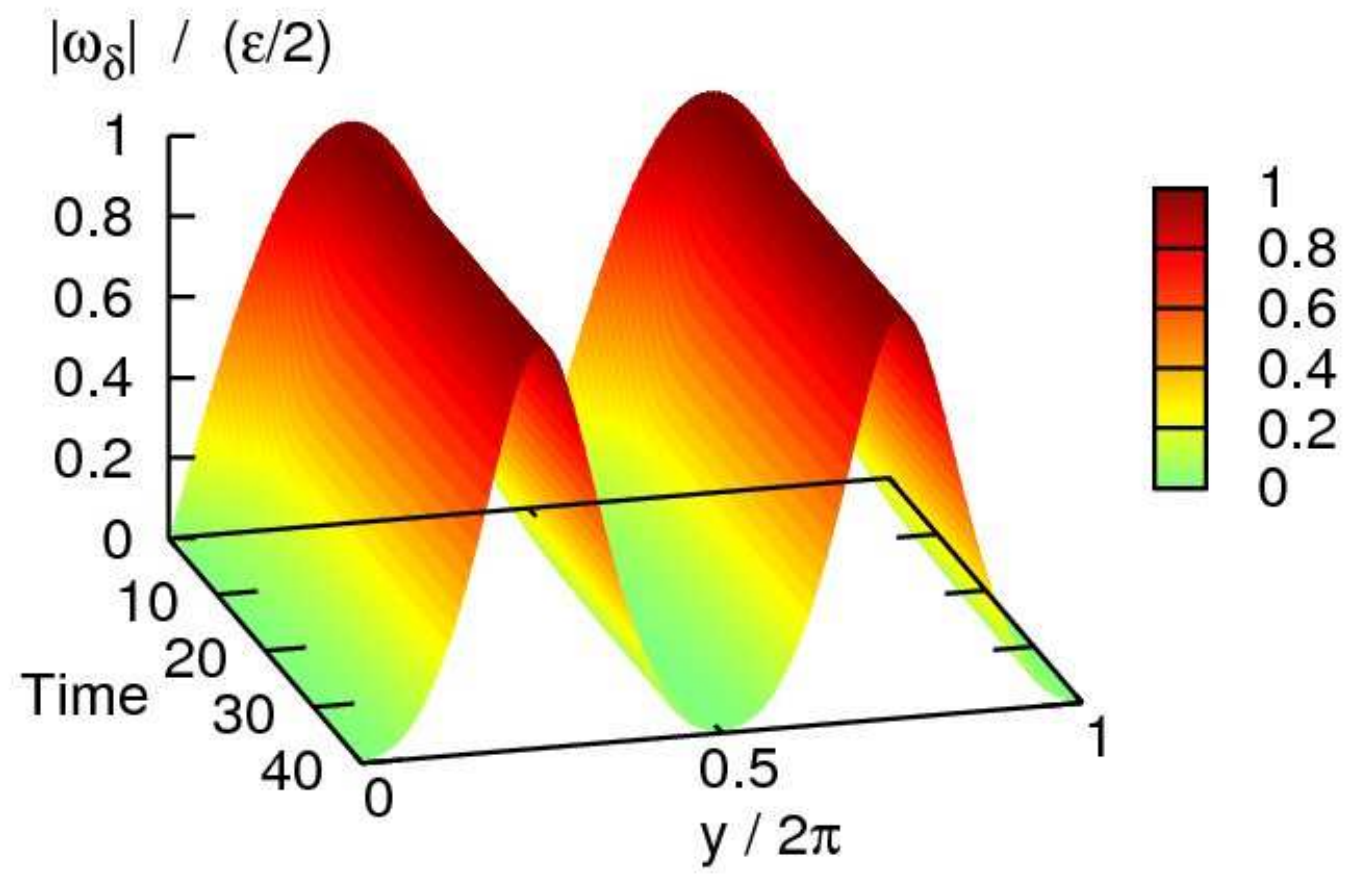

Figure 14: The space-time series of the modulus of the perturbation vorticity, $\left|\omega_{\delta}(y, t)\right|$, with initial perturbation profile $A(y)=\sin y$, and aspect ratio $\delta=1.1$. 


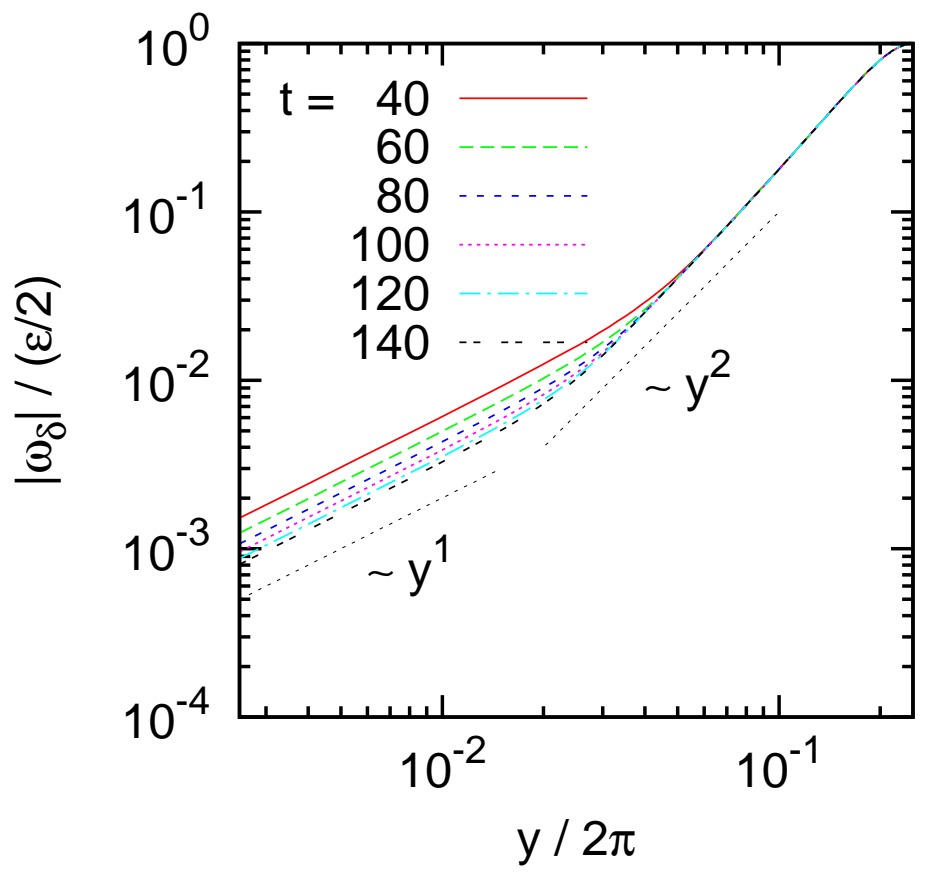

Figure 15: The profiles of the perturbation vorticity modulus, $\left|\omega_{\delta}(y, t)\right|$, at several times, for the initial perturbation profile $A(y)=\sin y$ and aspect ratio $\delta=1.1$. As the time goes on, the linear region becomes narrower and narrower, and instead the region with the profile proportional to $y^{2}$ extends towards the stationary streamlines, leading to a parabolic profile in the large time limit.

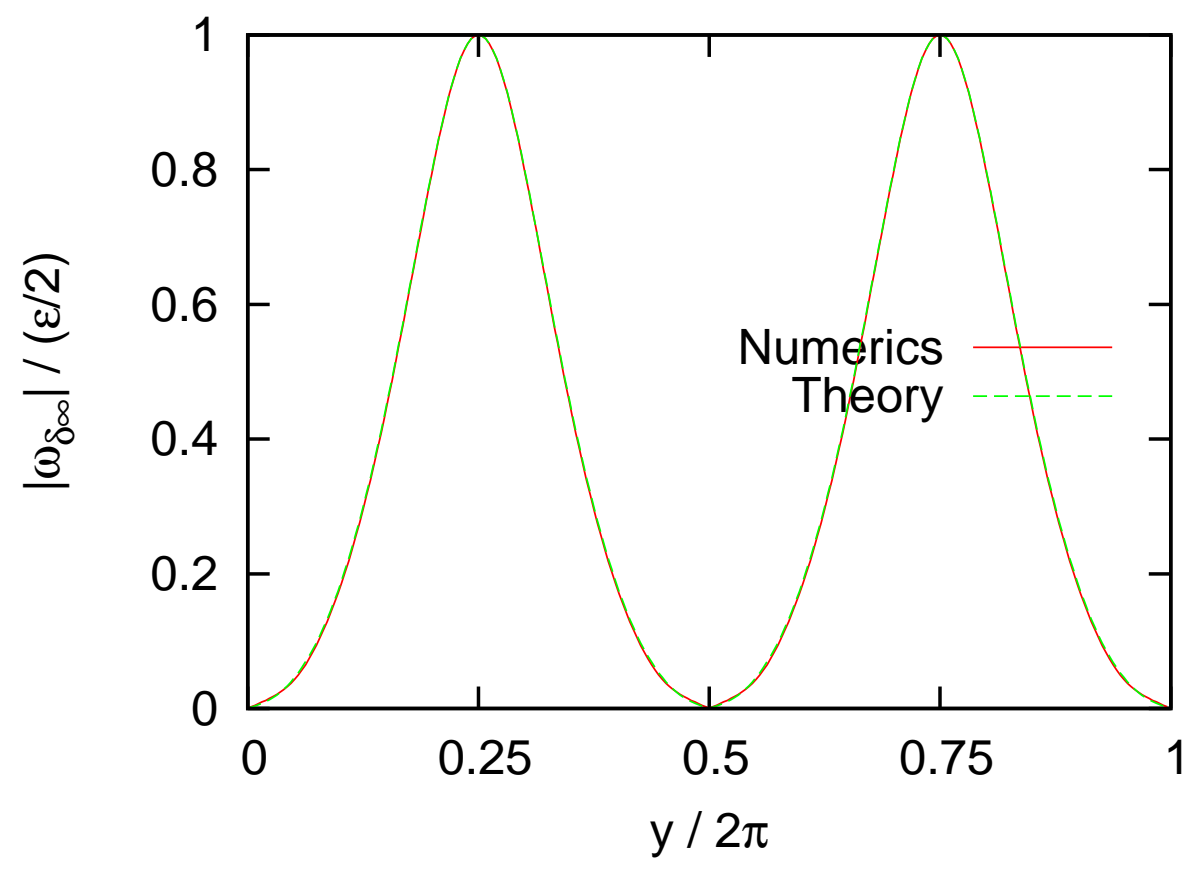

Figure 16: The final profile of the perturbation vorticity modulus, $\left|\omega_{\delta \infty}(y)\right|$, obtained from both direct numerical simulation and theory. The initial perturbation profile is $A(y)=\sin y$ and the aspect ratio is $\delta=1.1$. The two profiles show excellent agreement. 
(a)
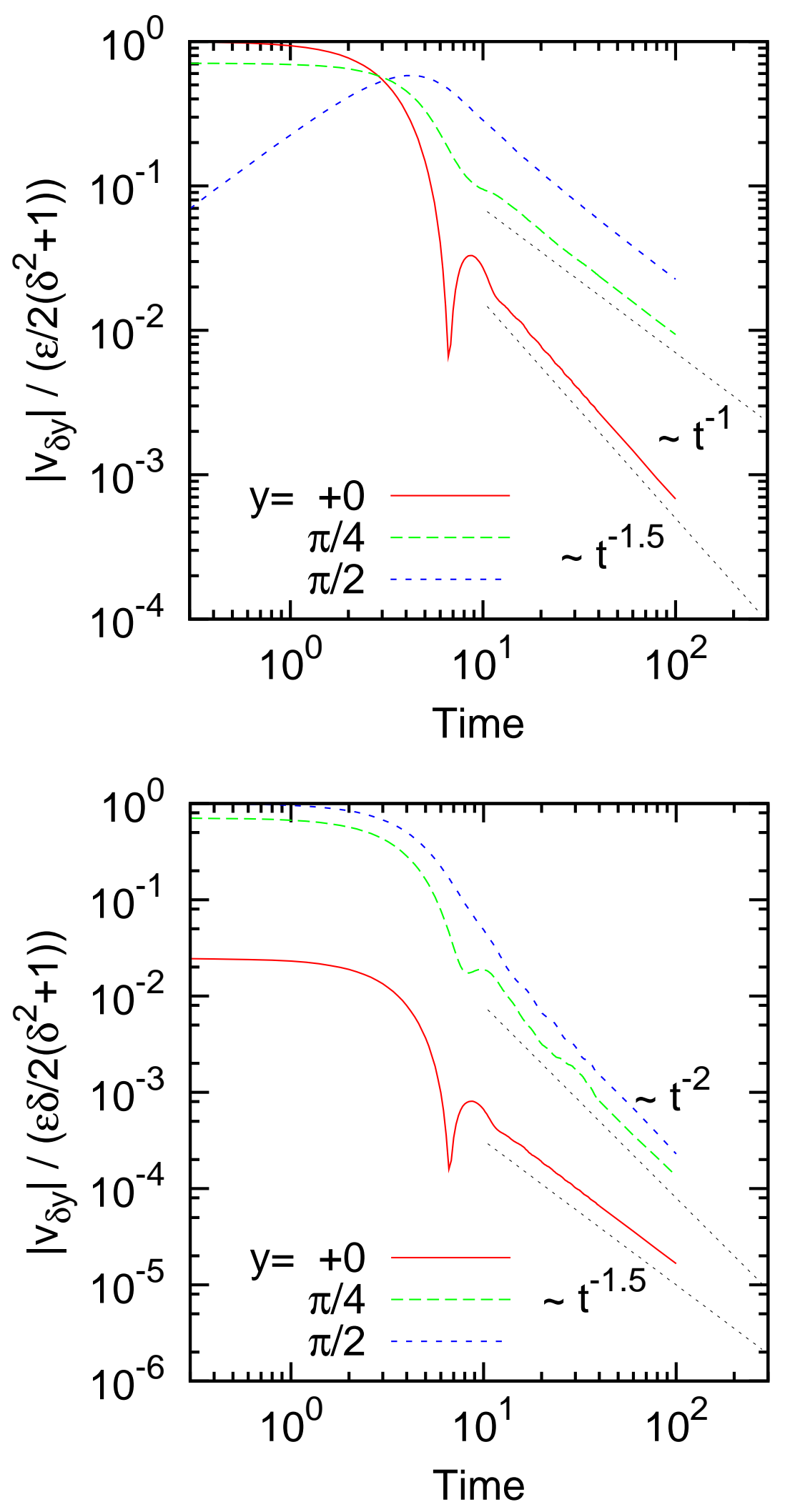

Figure 17: The time series of the perturbation velocity components, $\left|v_{\delta, x}(y, t)\right|(\mathrm{a})$ and $\left|v_{\delta, y}(y, t)\right|$ (b) at three locations, $y=0$ (vicinity of the stationary streamline) (red), $y=\pi / 4$ (green), and $y=\pi / 2$ (blue), for the initial perturbation profile $A(y)=\sin y$ and aspect ratio $\delta=1$.1. We observe the asymptotic forms $\left|v_{\delta, x}(y, t)\right| \sim t^{-\alpha}$, with $\alpha=1$, and $\left|v_{\delta, y}(y, t)\right| \sim t^{-\beta}$, with $\beta=2$, in the almost all the region, in accordance with the theory. Only in the vicinity of $y=0$ and $\pi$, we observe the exponents $\alpha=1.5$ and $\beta=1.5$, for which we have no theoretical predictions. 


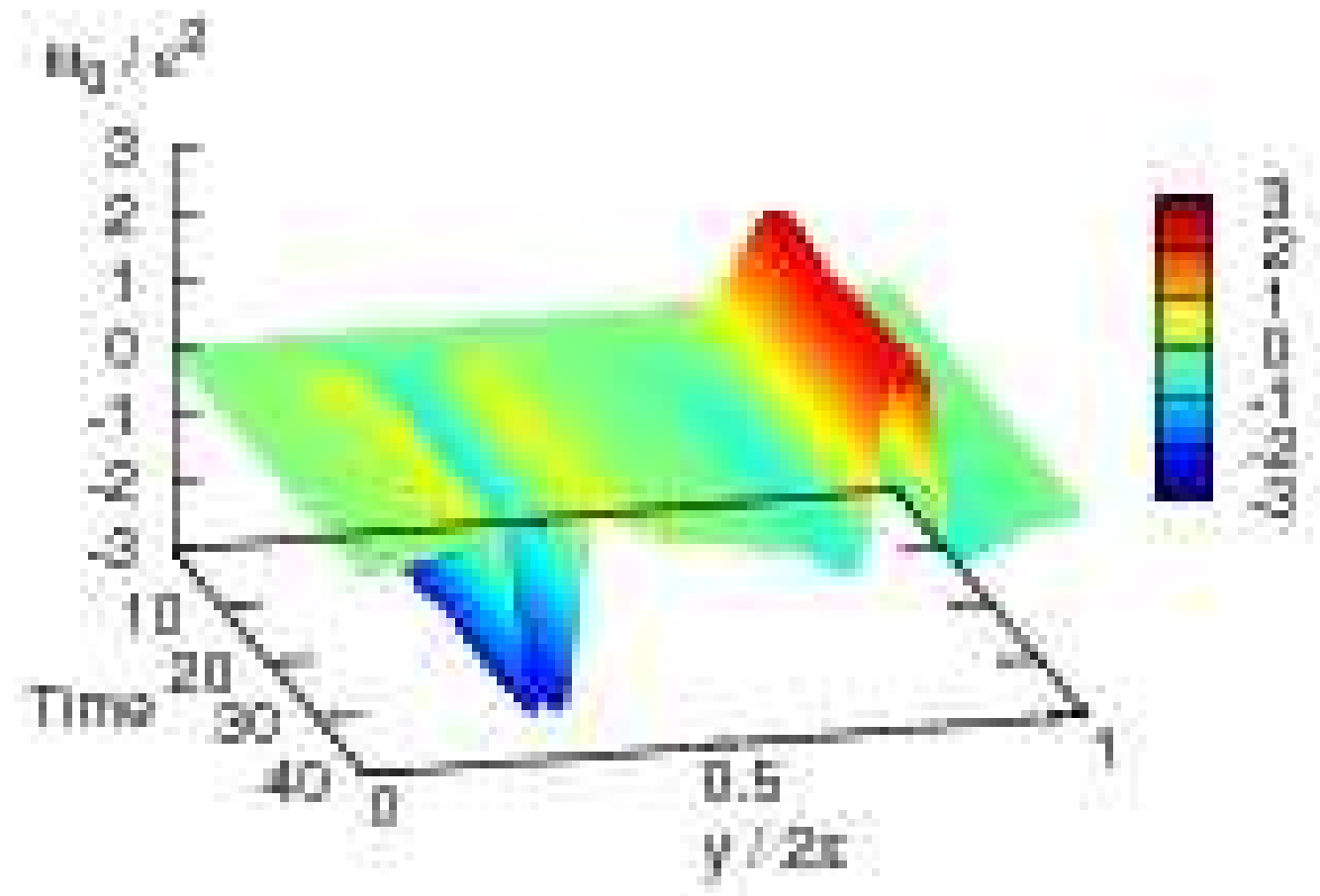

Figure 18: The space-time series of the $x$-averaged perturbation vorticity, $\omega_{0}(y, t)$. The initial perturbation profile is $A(y)=1$ and the aspect ratio is $\delta=1.1$. 


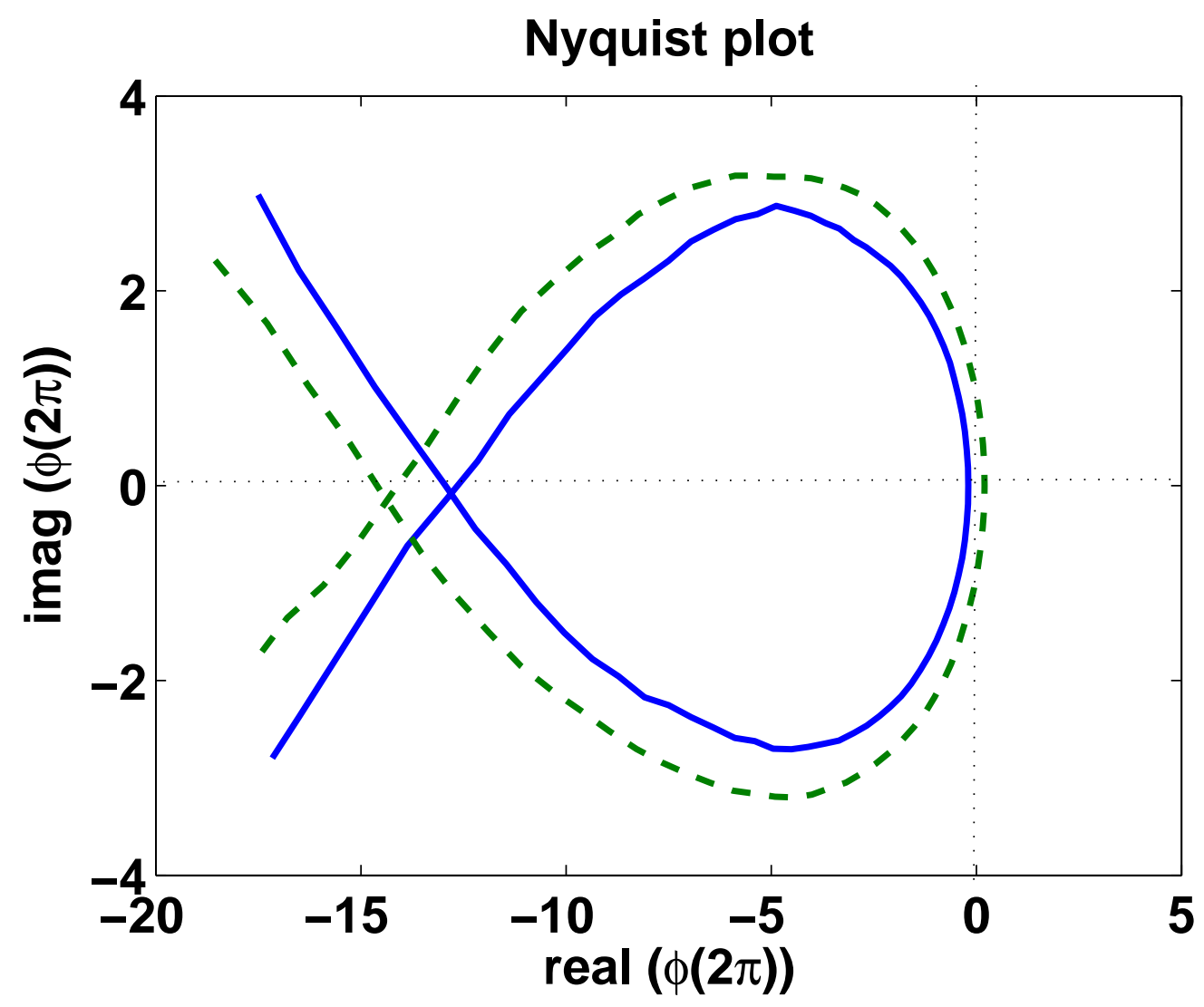




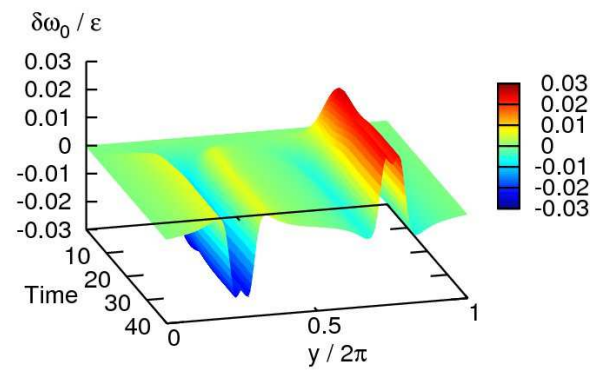


Minimum of the dispersion relation for neutral modes

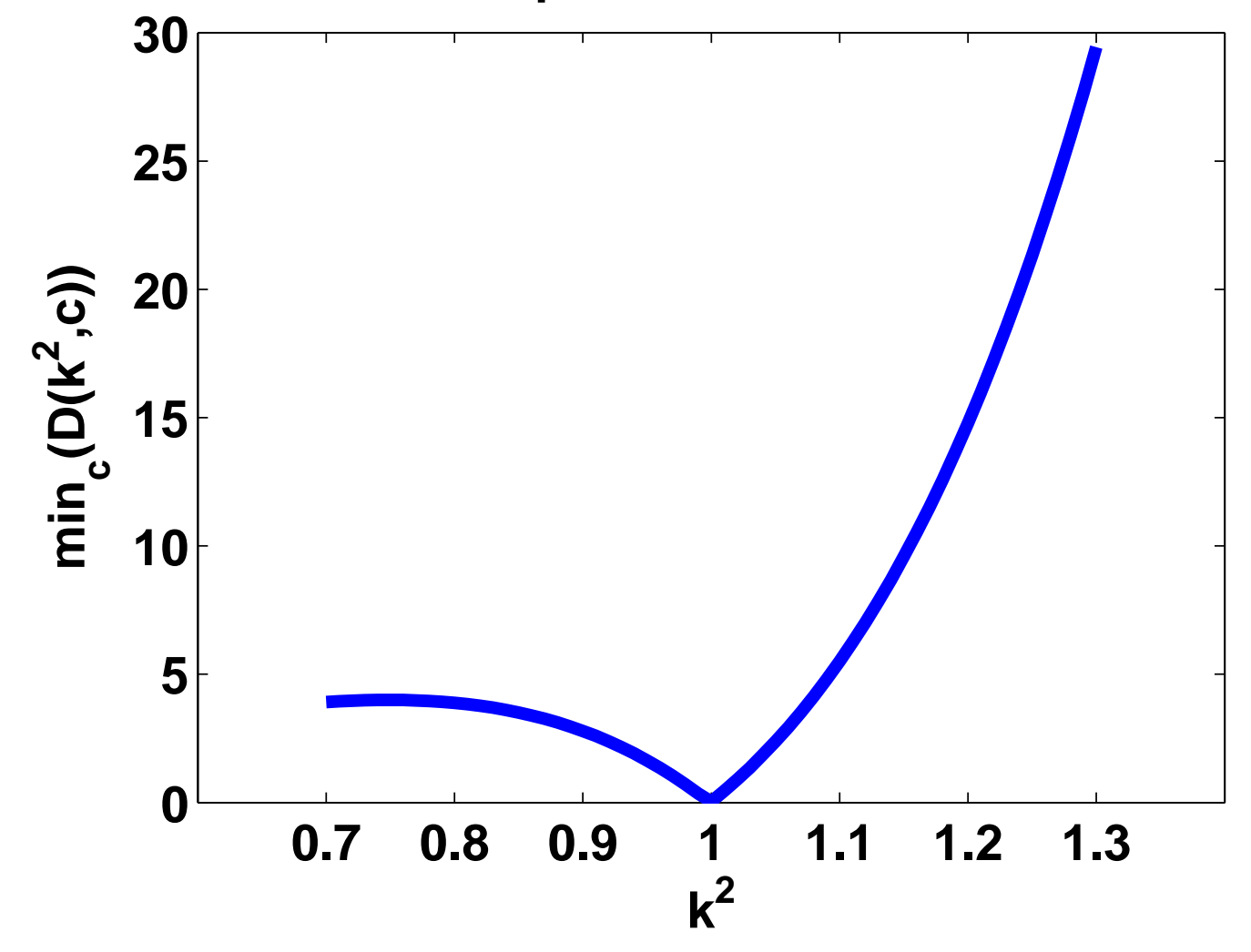




\section{Minimum of the dispersion relation for neutral modes}

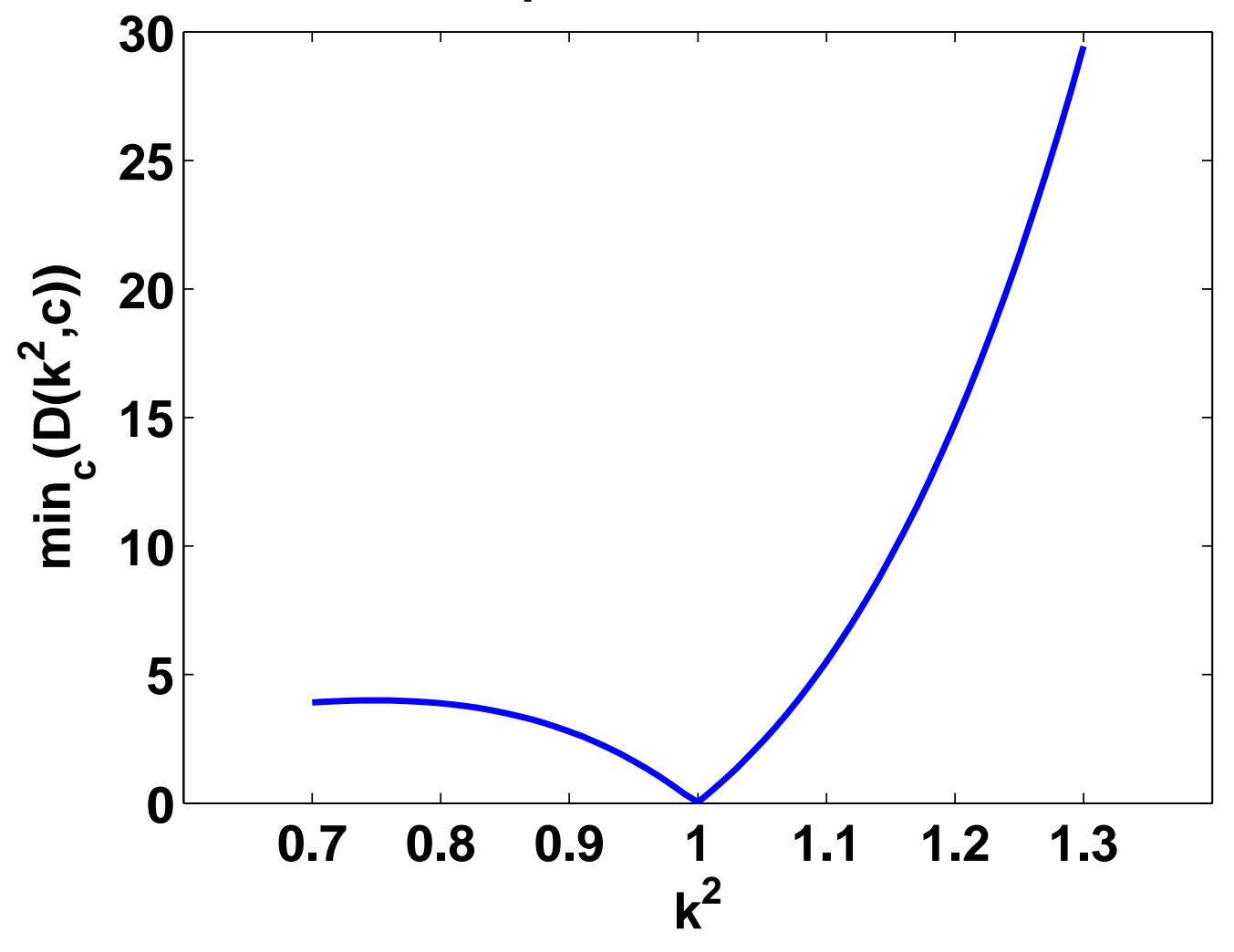




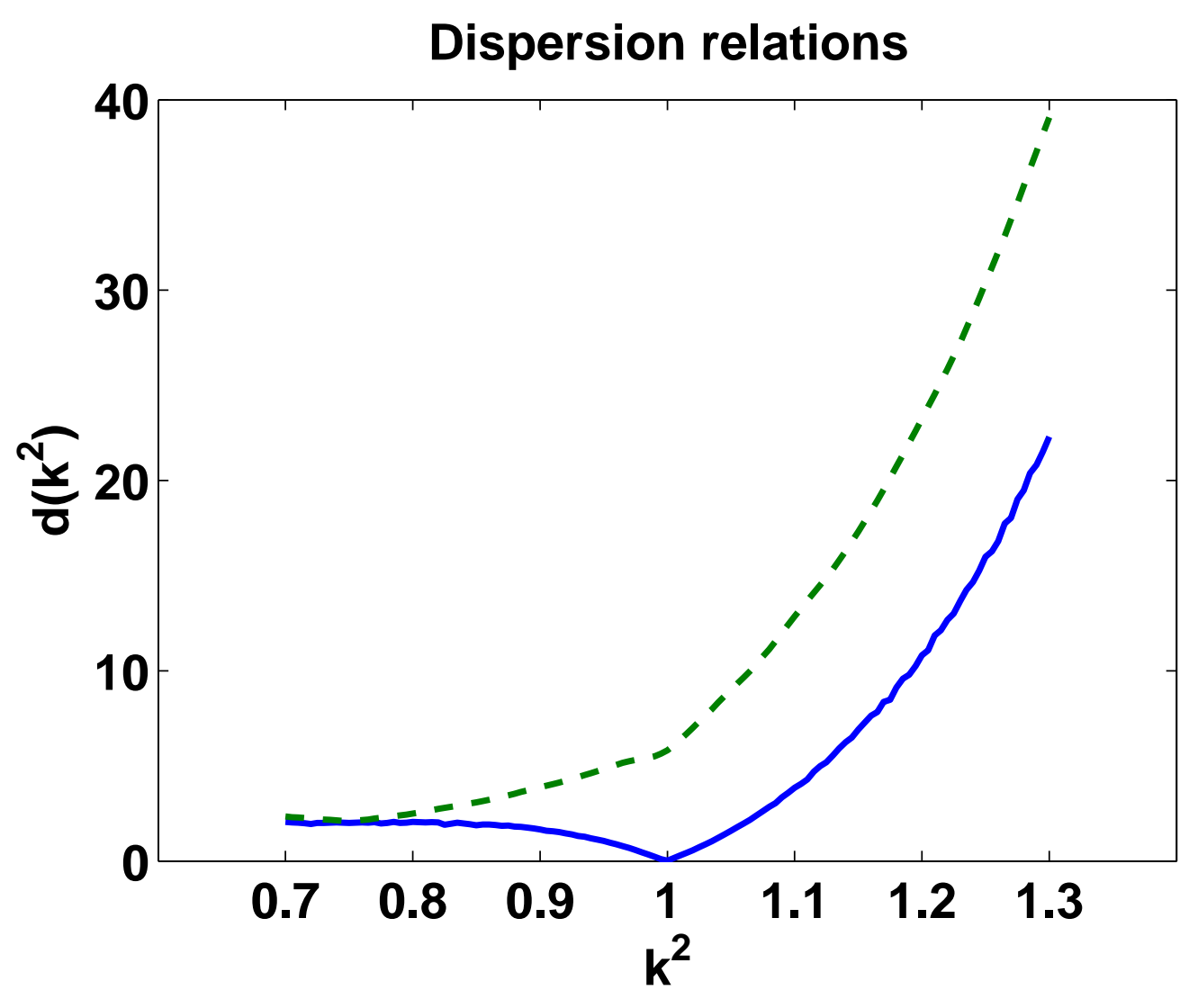




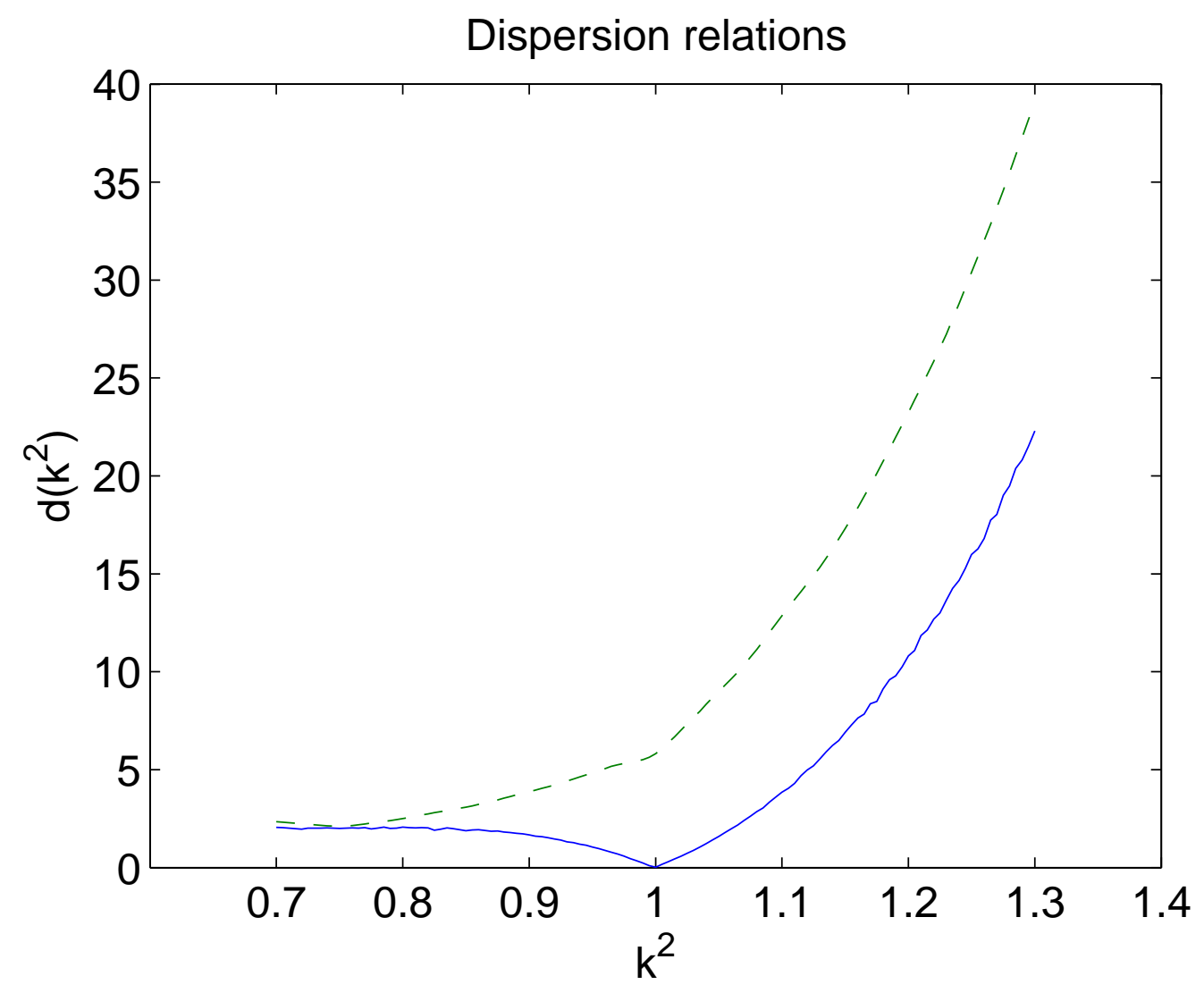




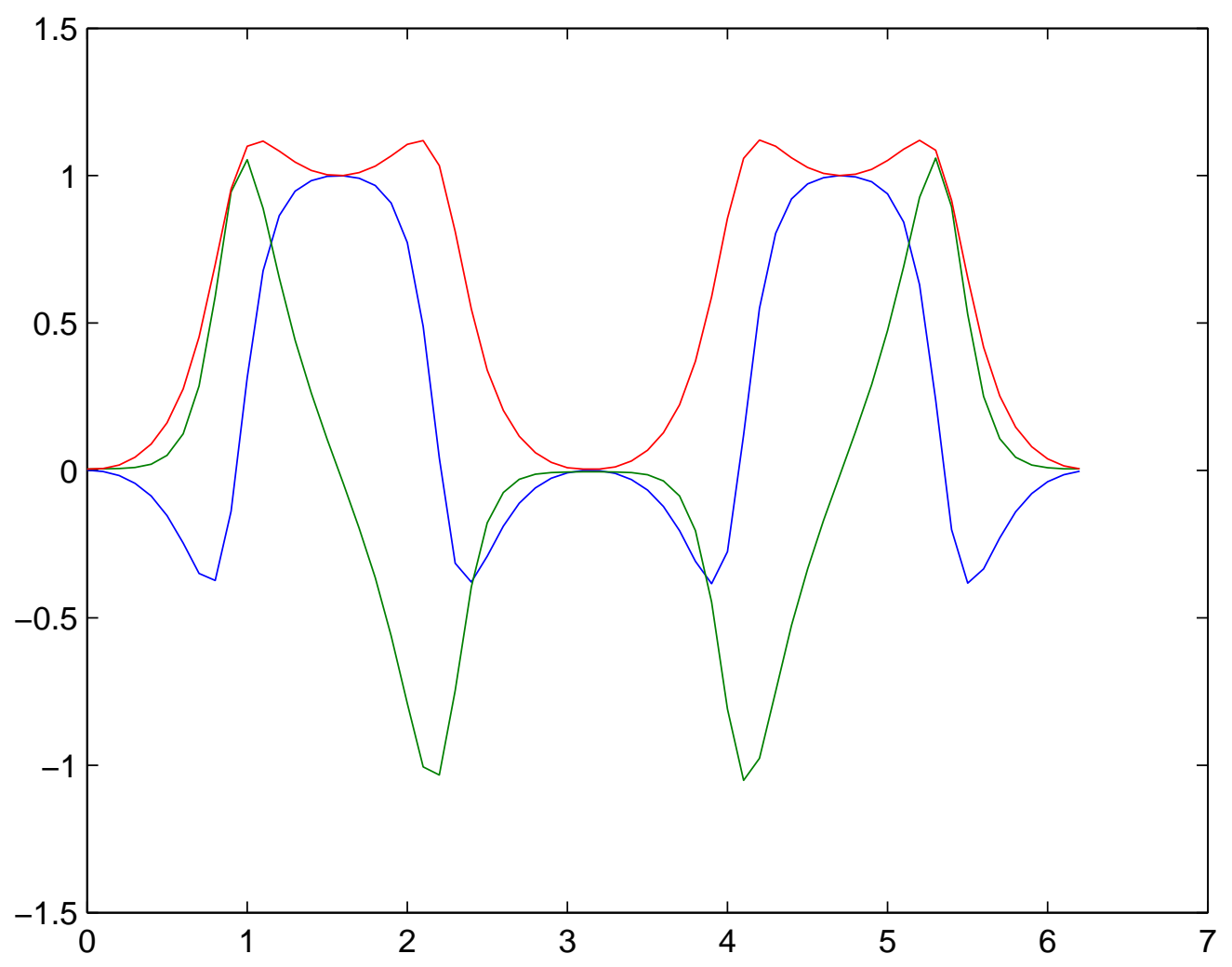

